\title{
SYNTHESIS, CHARACTERIZATION, AND APPLICATION OF WATER-SOLUBLE AND EASILY REMOVABLE CATIONIC PRESSURE SENSITIVE ADHESIVES
}

\author{
Final Report to DOE \\ Program/Project Identification No. DE-FC36-99GO10379
}

February 2003

PI: Prof. Yulin Deng, Institute of Paper Science and Technology 404894 5759, yulin.deng@ipst.edu

Co-PI: Prof. F. Joseph Schork, School of Chemical Engineering, Georgia Institute of Technology

404894 2866, joseph.schork@che.gatech.edu

Co-PI: Prof. Charles Q. Yang, School of Textile and Fiber Science, University of Georgia 706542 4912, cyang@hestia.fcs.uga.edu

Industry Partner: Dr. Ian McLennan, Ecosynthetix Inc. 5173364657, mclennan@ecosynthetix.com 


\section{TABLE OF CONTENTS}

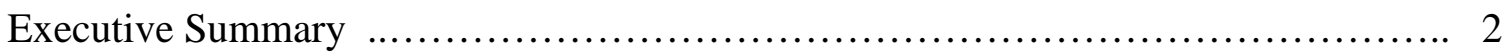

General Introduction ........................................................... 4

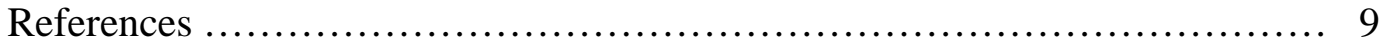

Part 1. Water-Soluble/Dispersible Cationic Pressure-Sensitive Adhesive (PSA) from solvent polymerization ........................................... 11

1.1. Summary …............................................................ 11

1.2. Synthesis and Characterization of Cationic Copolymers .................... 12

1.3. PSA Properties and Recyclability .................................. 26

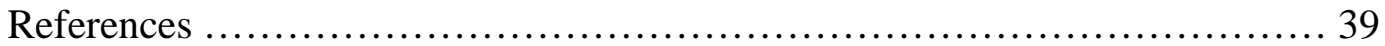

Part 2. Water-Soluble/Dispersible Cationic Pressure-Sensitive Adhesive (PSA) from Emulsion Polymerization .......................................... 40

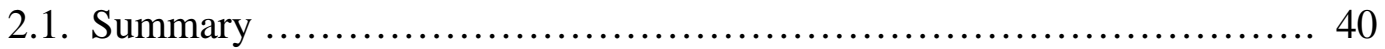

2.2. Emulsion Polymerization of Butyl Acrylate with the Cumene Hydroperoxide/Tetraethylenepentamine Redox Initiator .................. 41

2.3. Emulsion Copolymerization of Butyl Acrylate with Cationic Monomer Using an Interfacial Redox Initiator System ............................. 57

2.4. Water-Based Cationic PSA ........................................ 82

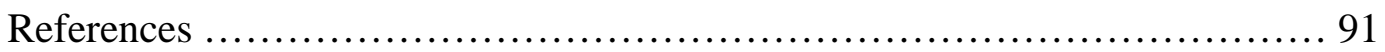

Part 3. Removal of Contaminants from Papermaking System ......................... 93

3.1. Removal of Contaminants through Fixation ............................ 94

3.2. Removal of Contaminants through Flotation ............................. 98

3.3. Conclusions .......................................................101

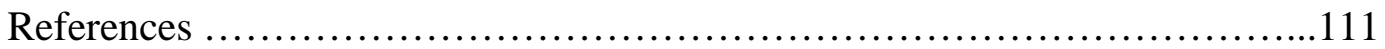




\section{EXECUTIVE SUMMARY}

This program is a joint effort of Institute of Paper science and Technology, Georgia Institute of Technology and University of Georgia at Athens to develop water-soluble and removable pressure sensitive adhesives.

The project started on October 1, 1998 and finished on December 30 ${ }^{\text {th }}, 2002$ after noncost extension.

The objectives of the original proposal are:

1. Synthesize water-soluble and easily removable cationic polymer resins for PSAs.

2. Optimize the PSA formulation for labels and tapes, and examine the end-use properties.

3. Study the colloidal properties of PSAs in water.

4. Study the repulpability of novel PSAs.

5. Examine the effect of adsorbed PSA (in a molecular or colloidal form) on papermaking operation and final paper properties.

After 4 years study, we accomplished all proposed objectives. One patent application, "Water-Soluble/Dispersible and Easy Removable Cationic Adhesives and Coating For Paper Recycling” was filed on July 2, 2000 (Application number: 09/621,695). Another 5 papers were published or submitted to different scientific journals.

The main conclusions from this study are summarized as the following:

1. The cationic monomers can be copolymerized into polyacrylate that is one of the most important polymers for pressure sensitive adhesive (PSA) using different technologies, including solvent polymerization and miniemulsion polymerization.

2. The copolymers of cationic acrylate can be formulated to pressure sensitive adhesives with excellent end-use properties.

3. The cationic PSA is water-soluble or dispersible if the cationic content is higher than 10 mol\%. However, if the cationic content is too high (>20\%), the glass 
transition temperature of the PSA increases resulting in the decrease of the tackiness and the peel strength of the PSA.

4. The water-soluble or dispersible cationic PSAs will adsorb to fibers and be removed from pulp furnish in papermaking process. Therefore, they will not cause stickies problem on a paper machine.

5. $95 \%$ of dispersed/water-soluble PSAs will adsorb on fiber surfaces even for the white water that are reused for more than 15 cycles.

6. The adsorption of soluble/dispersible PSAs on wood fibers will not affect fiber properties.

7. In average, the cost of novel PSA is about 3\% higher than current commercially available PSA. However, the PSA developed in this study is recyclable. 


\section{GENERAL INTRODUCTION}

In recent years, the world has expressed an increasing interest in the recycling of waste paper to supplement the use of virgin fiber as a way to protect the environment. Statistics show that major countries are increasing their use of recycled paper. For example, in 1991 to 1996, the U.S. increased its recovered paper utilization rate from 31\% to 39\%, Germany went from $50 \%$ to $60 \%$, the UK went from $60 \%$ to $70 \%$, France increased from $46 \%$ to $49 \%$, and China went from $32 \%$ to $35 \%$ [1]. As recycled fiber levels and water system closures both increase, recycled product quality will need to improve in order for recycled products to compete with products made from virgin fiber [2]. The use of recycled fiber has introduced an increasing level of metal, plastic, and adhesive contamination into the papermaking process which has added to the complexity of the already overwhelming task of providing a uniform and clean recycle furnish. The most harmful of these contaminates is a mixture of adhesives and polymeric substances that are commonly known as stickies.

Stickies, which enter the mill with the pulp furnish, are not easily removed from the repulper and become more difficult the further down the system they get. This can be detrimental to the final product quality. Stickies are hydrophobic, tacky, polymeric materials that are introduced into the papermaking system from a mixture of recycled fiber sources. Properties of stickies are very similar to the fibers used in papermaking, viz. size, density, hydrophobicity, and electrokinetic charge. This reduces the probability of their removal by conventional separation processes, such as screening and cleaning, which are based on such properties. Also, their physical and chemical structure allows for them to extrude through screens, attach to fibers, process equipment, wires and felts. Stickies can break down and then reagglomerate and appear at seemingly any place in the mill. When subjected to a number of factors including changes in $\mathrm{pH}$, temperature, concentration, charge, and shear forces, stickies can deposit [3]. These deposits can lead to decreased runnability, productivity and expensive downtime. If the stickie remains in the stock, then machine breaks can be common. Finally, if the stickie is not removed or 
deposited, it will either leave in the final product causing converting and printing problems or recirculate within the mill.

It has been estimated that stickies cost the paper industry between $\$ 600$ and $\$ 700$ million a year due to the cost of control methods and lost production attributed to stickies [3]. Also, of the seven recycling mills opened in the United States between 1994 and1997, four have closed citing stickies as the main reason responsible for the closure [4].

Adhesives are widely used throughout the paper and paperboard industry and are subsequently found in the recycled pulp furnish. Hodgson stated that even the best stock preparation process can only remove $99 \%$ of the contaminants, of which the remaining $1 \%$ is usually adhesives of various types which are usually10-150 microns in effective diameter [5]. The large particles are removed by mechanical means such as cleaners and screens, and the smaller, colloidal particles can be removed with washing. The stickies that pass through the cleaning and screening processes cause 95\% of the problems associated with recycling [6]. The cleaners will remove most of the stickies that have a density varying from the pulp slurry $\left(\sim 1.0 \mathrm{~g} / \mathrm{cm}^{3}\right)$ and will accept stickies with densities ranging from $0.95-1.05 \mathrm{~g} / \mathrm{cm}^{3}$ [2]. The hydrophobicity of the material is also an important characteristic of the stickie [7]. The hydrophobicity causes the stickies to agglomerate with other hydrophobic materials such as other stickies, lignin, and even pitch. The tacky and viscous nature of stickies contributes to many product and process problems, negatively affecting the practicality of recycled fiber use.

The source of stickies that evade conventional removal techniques are usually synthetic polymers, including acrylates, styrene butadiene rubber, vinyl acetates, and polypropylene [5,6,8-12]. Sources of these adhesives are usually broken down into categories based on application. These categories include contact and pressure sensitive adhesives, PSAs, and binders as illustrated [13]: 


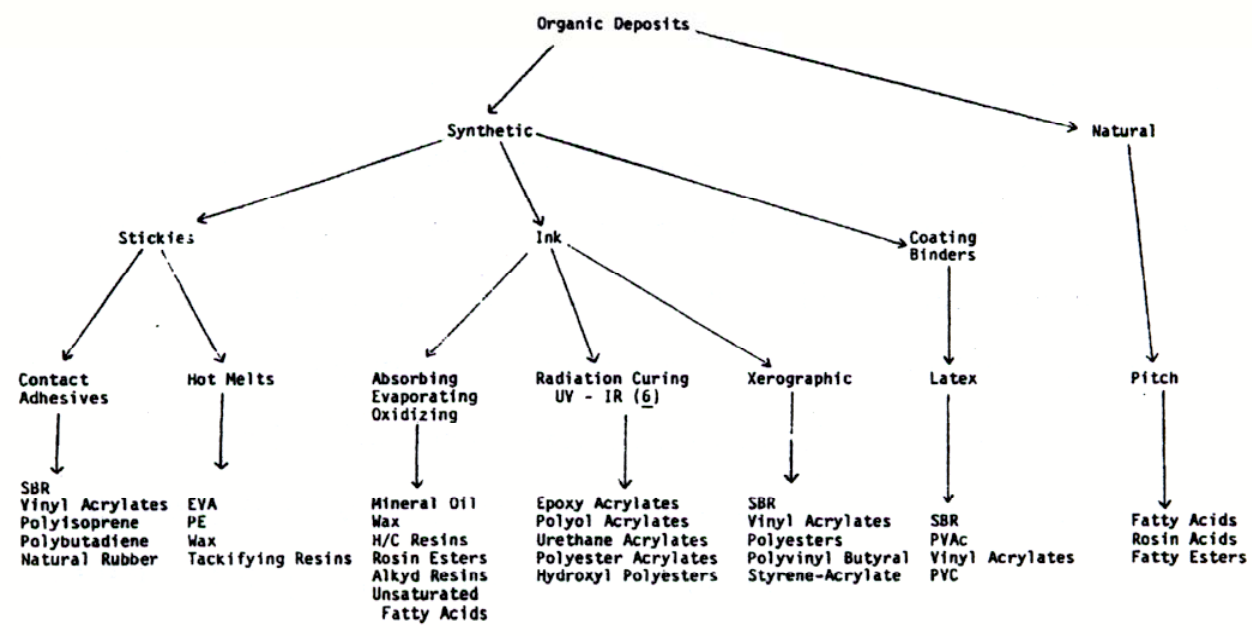

Pressure sensitive adhesives are primarily made up of a polymer, such as polyacrylate, styrene butadiene rubber (SBR) and a tackifying agent. PSAs are commonly used in selfsealing envelopes, tapes and labels and the tackiness of PSAs is not temperature sensitive. PSAs commonly contain ethyl vinyl acetate (EVA) or polyvinyl acetate (PVA) as a backbone and a tackifying resin. They are mainly used in book and magazine bindings, boxes, envelopes, bags, and tubes. PSAs are sensitive to temperature. Several studies have collected deposits from mills and have showed the composition of these tacky deposits to commonly be acrylates and polyvinyl acetates [14-20].

The first step to controlling stickies would be to prevent them from entering the mill in the first place. However, this is not an easy task. Problems of stickies can be prevented by careful inspection of the wastepaper entering the mill [21]. Many recycle mills employ people to remove some of the waste paper that has visible stickie contaminants, to avoid process problems [22]. Contaminated waste paper that is removed is sent to the landfill, unused, at the expense of the mill.

Screening and cleaning currently remove the majority of stickies. Macro stickies are effectively screened out with fine screens, however, as the slots in screens are made smaller, fiber loss occurs. Cleaners separate based on the difference in stickie density and pulp density [6]. After cleaning and screening, stickies are as small as $0.15 \mathrm{~mm}$ and 
can still be seen in the final product, even if they do not reagglomerate. Dispersion may break up the stickies further, but that is very dependent on consistency, pressure and temperature.

By taking advantage of stickies chemical properties that allow for stickies to elude cleaners and screens, several control strategies have been employed. Talc is a common chemical additive that is used to pacify stickies by covering stickie surfaces, causing them to be less tacky, increasing the specific gravity and allowing for easier removal by cleaners [23.] Talc's surface energy is lower than most adhesives, increasing its attraction to stickies. Other chemicals used for stickies control include dispersants, solvents, cationic polymers, synthetic fibers, zirconium compounds and alum sequestering agent [6]. However, additives are usually very expensive and do not solve the problem, they only temporarily fix it. Talc, zirconium compounds, and synthetic fibers only stabilize stickies by detackifying them, they do not reduce the particle size. These complexes are also shear sensitive, which can cause additional stickie surfaces to be exposed. Dispersants will reduce the particle size and prevent reagllomeration but are temperature and $\mathrm{pH}$ sensitive, which will cause compatibility problems with the existing mill chemistry and raised environmental issues. Anionic stickies are usually countered with low molecular weight, highly charged cationic polymers.

Bruyns et al. have studied problematic stickie deposits at a recycle mill [22]. The mill initially used caustic to clean the wires, which then caused an increase in the use of other chemicals, such as defoamer, sizing and anti-skid agent. It is also known that defoamer is sometimes a component of stickie deposits, thus aiding in stickie deposition on equipment. Other chemical usages were also altered in the mill. Ultimately, the mill returned to manual removal of most contaminated waste paper before pulping to save on further chemical usage.

Hawes [24] has studied a number of machine clothing types to minimize the deposition of stickies. Optimal shower placement and fabric resistance to chemicals that are used to clean off contaminants is where most of this work has been done. However, fabric 
coating that is resistant to stickie build up frequently wears off within a few days due to usage. Low molecular weight, high cationic charge density polymers are also applied to the machine wire as a barrier to prevent deposition. Cleaning solvents can also be used on machine clothing but cannot be returned to the process due to the high contamination and favorable stickie interaction.

In summary, after twenty years of paper recycling experience, stickies remain one of the major issues in recycled paper. Most of the stickie removal techniques are concentrated on physical properties of the stickie. Most stickie control strategies concentrated on passivation, by addition of chemicals such as talc, and coagulation and dispersion, by polymers. Stickie-surface interactions have concentrated on felts, fabrics, and rolls. None of these techniques are $100 \%$ efficient at removing stickies from the recycle system. Therefore, there is need to develop new technology to solve the stickies problem. Many researchers have focused on the understanding and resolution of the stickies problems in waste-paper recycling and papermaking. Two approaches have been made to solve stickies associated problems, i.e. sticky control and repulpable sticky development. Although sticky problems can been reduced by one or more of the techniques listed above, the techniques that work well in one paper mill may not work for others because of the differences in the adhesives, pulps, and papermaking conditions. Obviously, to control the deposition of stickies on the paper machine and paper sheets is a passive rather than an initiative method because it can only reduce but cannot totally solve the sticky problems. Therefore, the development repulpable PSAs is of high interest to the paper industry.

The technologies currently being evaluated by adhesive manufacturers in an effort to make repulpable. The recoverable PSAs commonly have a high glass transition or melting temperature, and will not soften and breakdown under repulping conditions. Although this is a good approach, only limited thermoplastic raw materials can be used as a PSA. Cross-linked thermoset PSAs have been developed using polyurethane-based polymers. These PSAs have shown significant advantages over thermoplastic PSAs, but the energy required for repulping is higher, and the crosslinking of the PSAs during the 
storage and operation is a problem. Another approach for recoverable PSA is to use polymers with a density significantly lower than or higher than the fibers. However, the deformation of these soft particles and their tacky property lead to a low separation efficiency. In contrast to the recoverable PSAs, PSAs that can be dissolved or dispersed in water have been developed. These PSAs include vinyl polymers modified with hydrophilic components such as starch, carboxylic acids, polyglycols, maleic acid, polyethylene oxide, etc. The disadvantages of these water-soluble/dispersible PSAs include poor heat stability and water resistance, and low bonding strength. An anionically charged water-soluble PSA was developed by Eastman Chemical Co. recently. Although the anionic PSA has good heat stability and high bonding strength, the accumulation of water-soluble anionic PSA in the process water is a serious problem. Therefore, a water-soluble PSA that has a good bonding strength, high stability, and will not accumulate in water was focused in this program. Because these novel PSAs are water soluble, they will not form stickies in pulp. Furthermore, because the PSAs are cationically charged, they can be easily removed from the system by adsorbing onto fiber and fines surfaces. As a result, the "stickies" will no longer be a problem in recycled paper mills. Because paper made up $40 \%$ of the municipal solid waster, and the annual cost of stickies to the paper industry is estimated to be about $\$ 600$ millions, a full solution of stickies problems will significantly improve our environment and save $\$ 600$ millions per year.

\section{References}

1. H. Sasaki, T. Shibano and M. Yamakage, Packaging Technology and Science, 11, 205 (1998).

2. O. Heise, M. Kemper, H. Wiese, E. Krauthauf, Tappi J., 83(3), 73 (2000).

3. T. Friberg, Progress in Paper Recycling, Nov, 70 (1996)

4. D. Cauchon, USA Today, Oct. 7, 1997. P.3A.

5. K. Hodgson, Paper Recycling Challenge- Stickies Vol. 1, 71 (1997).

6. T. Fogarty, Pulping Conference Proceedings, Book 2, Boston, MA, 429 (1992).

7. P. Alison, Paper South Africa. 12(5), 47 (1992). 
8. R. Diaz, IPST Master's Thesis. Atlanta, GA, June (1995).

9. W. Scholz, Pulping Conference Proceedings, Atlanta. Book 2, 501 (1993).

10. N. Hsu, J. Schroeck and L. Errigo, Recycling Symposium Proceedings. Chicago, IL, 331 (1997).

11. M. Douek, Paper Recycling Challenge- Stickies Vol. 1, 15 (1997).

12. T. Ling, TAPPI Pulping Conference Proceedings. TAPPI Press, Atlanta, GA, 1039 (1991).

13. R.D. Moreland, TAPPI Pulping Conference Proceedings. TAPPI Press, Atlanta, GA.,193 (1986).

14. C.R. Olsen, and M.K. Letscher, Appita Journal, 45(2), 125 (1992).

15. N. Hsu, Paper Recycling Challenge- Stickies Vol. 1, 256 (1997).

16. L. Latimer, Tappi J., 62(5), 29 (1979).

17. D. Wilhelm, S. Makris and S. Banerjee, Tappi J., 82(12), 63 (1999).

18. S. Patel and S. Banerjee, Tappi J. 82(11), 99 (1999).

19. R. Venditti, H. Chang and H. Jameel, Paper Age, Nov, 18 (1999).

20. K. Cathie, R. Haydock and I. Dias, Pulp and Paper Canada. 93(12), 157 (1992).

21. M.R. Doshi, Progress in Paper Recycling, Nov, 54 (1991).

22. M. Bryuns, K. Rajan, R. Dayliss, and S Van Doren, Paper Recycling ChallengeStickies, Vol. 1, 292 (1997).

23. J. Yordan and G. Williams, Paper Recycling Challenge-Stickies, Vol. 1, 76 (1997).

24. J. Hawes, Paper Recycling Challenge-Stickies, Vol. 1, 259 (1997). 


\section{PART 1. WATER-SOLUBLE/DISPERSIBLE CATIONIC PRESSURE- SENSITIVE ADHESIVES FROM SOLVENT POLYMERIZATION}

\subsection{Summary}

In order to solve stickies problems, a series cationic copolymers of butyl acrylate (BA) and [3-(methacryloylamino)-propyl]trimethylammonium chloride (MAPTAC) were synthesized by free radical solution polymerization in methanol or ethanol. FT-Raman and nuclear magnetic resonance (NMR) were applied to monitor the polymerization process. The copolymers were characterized by light scattering, NMR, differential scanning calorimetry (DSC), and thermogravimetric analysis (TGA). It was found that random copolymers could be prepared, and the molar fractions of butyl acrylate and cationic monomers in the copolymers were close to the feed ratios. The copolymer prepared in methanol has higher molecular weight than that prepared in ethanol. With increased cationic monomer content, the glass transition temperature $\left(\mathrm{T}_{\mathrm{g}}\right)$ of the copolymer increases, while the thermal stability decreases. The reactivity ratios for the monomers were evaluated. Copolymerization of BA $\left(\mathrm{M}_{1}\right)$ with MAPTAC $\left(\mathrm{M}_{2}\right)$ gave reactivity ratios as $r_{1}=0.92$ and $r_{2}=2.61$ in ethanol, and $r_{1}=0.79$ and $r_{2}=0.90$ in methanol. The cationic butyl acrylate (BA) and [3-(methacryloylamino)propyl] trimethylammonium chloride (MAPTAC) copolymers synthesized in ethanol were used as PSAs. The PSA water-solubility, end-use properties, repulpability in paper recycling, and its effect on the properties of recycled paper product were studied. It was found that the cationic PSAs can be dissolved or dispersed in water by controlling the cationic charge density in the backbone of PSAs; therefore, they will not deposit as stickies during recycling and papermaking processes. Because the PSAs are cationic charged, they can be easily removed from the papermaking system by adsorbing onto the negatively charged fiber and fine surfaces. Furthermore, the adsorbed colloidal or dissolved PSAs have little effect on final paper properties. 


\subsection{Synthesis and Characterization of Cationic Copolymers}

Synthesis of copolymers containing both hydrophobic and cationic hydrophilic units has been studied intensively. Emulsion polymerization techniques have been applied to synthesize cationic polymeric latexes, which have shown potential applications as catalysts [1,2], papermaking aids [3-6], and coating agents [7]. However, there are several problems in using emulsion polymerization techniques for preparation of this type of copolymer. First, because cationic monomers are strongly hydrophilic and are not miscible with the hydrophobic monomers in water, the incorporation rate of cationic monomers to the polymer through direct emulsion polymerization has been low. Brouwer [8] studied the emulsion polymerization of styrene (80-100 wt\%) and [2methacryloxy)ethyl]trimethylammonium chloride (MAETAC, 0-20 wt\%), and found that the maximum incorporation of MAETAC to the latex was less than 4 wt\%. Second, because the solubility of cationic monomers in water is significantly different from that of hydrophobic monomers, the emulsion copolymerization of these two different monomers usually results in two polymeric fractions, i.e., a high-cationic-monomercontaining copolymer and a high-hydrophobic-monomer-containing copolymer [9]. Third, emulsion polymerization cannot be used to prepare a high-cationic-containing copolymer because the copolymer will be water soluble and it cannot exist in a latex form in water if the cationic content is high.

Copolymers containing both hydrophobic and cationic hydrophilic units with a high cationic content have been synthesized by cationization of the copolymer that contained a functional monomer [2,10-13]. The copolymerization of the hydrophobic monomer and cationic monomer in an organic solvent has also been used to synthesize these types of copolymers [12-15]. These copolymers have potential application as adhesive curing agents [12], controlled drug release agents [14], and ion-exchange membranes [15]. Although copolymers with a high cationic content have already been made for various applications, the synthesis of these types of copolymers by solution polymerization has not been well addressed. In addition, it is our intention to develop a new class of cationically charged water soluble/removable pressure-sensitive adhesives. In current 
paper recycling industry, one of the serious problems is the stickies formed by pressure sensitive adhesives, which increases paper breaks and machine downtime. The cost of stickies for paper recycling industry is over $\$ 600$ million/year. The potential advantage of using cationically charged pressure sensitive adhesive is that the adhesive can be made to be water-soluble by controlling the charge density on the polymer backbone, and these water-soluble molecules can adsorb to wood fiber surface and be removed with paper web during paper making process. In this study, a series of poly(BA-co-MAPTAC) copolymers with different cationic content was synthesized by solvent polymerization and characterized. The effect of reaction conditions on the properties of the copolymers was studied, and the reaction kinetics was reported. The properties of these polymers as pressure-sensitive adhesives and their effect on paper recycling will be reported later.

When solution polymerization is used to prepare a homogeneous poly(BA-coMAPTAC), the choice of the right solvent is critical. First, both BA (hydrophobic) and MAPTAC (cationic and hydrophilic) should be soluble in the solvent used. Second, the solvent should also be a good solvent for the copolymer to prevent the precipitation of the copolymers. Third, both monomers should have reasonable monomer reactivity ratios in the solvent. Because both ethanol and methanol are strong polar organic solvents, it is expected that they can fit these requirements.

\section{Experimental}

\section{Materials}

All chemicals were purchased from Aldrich Chemical Company. Butyl acrylate (BA, 99+\%) and ethylene glycol dimethylacrylate (EGMA) were washed with 5\% sodium hydroxide aqueous solution three times and then with deionized water three times to remove inhibitors. [3-(Methacryloylamino)propyl]trimethylammonium chloride (MAPTAC, $50 \mathrm{wt} \%$ in water) was extracted with ethyl ether five times to remove inhibitors, and the residual ethyl ether was removed by a rotary evaporator at room 
temperature under reduced pressure. 2,2'-Azobisisobutyronitrile (AIBN), methanol, and ethanol were used as received. Solvents used were all HPLC grades.

\section{Polymer Synthesis}

Copolymers were synthesized by solution polymerization in methanol or ethanol. A typical example (Sample P3 in Table 1) is as follows: a 250ml three-neck round flask equipped with a thermometer, nitrogen inlet, condenser, and mechanical stirrer was charged with $25.6 \mathrm{~g}$ of BA $(0.20 \mathrm{~mol}), 7.8 \mathrm{~g}$ of MAPTAC (0.035 mol), $42.9 \mathrm{~g}$ of ethanol. The mixture was purged with nitrogen for 30 minutes and heated to $65^{\circ} \mathrm{C}$. After temperature reached equilibrium, $0.193 \mathrm{~g}$ of AIBN $(1.18 \mathrm{mmol})$ in about $5 \mathrm{ml}$ ethanol was injected. The mixture was kept at $65^{\circ} \mathrm{C}$ for 4 hours. After cool down, the mixture was poured into a large amount $(\sim 250 \mathrm{ml})$ of hexane with stirring, and then the bottom layer was washed repeatedly with hexane $(3 \times 100 \mathrm{ml})$. The purified polymer was dried first in air and then at $40^{\circ} \mathrm{C}$ for 24 hrs under vacuum.

\section{Characterization}

Raman spectra were recorded on a Nicolet 950 FT-Raman Spectrometer with an InGaAs detector to monitor the polymerization process. The resolution was $8 \mathrm{~cm}^{-1}$ and there were 200 scans for each spectrum. ${ }^{1} \mathrm{H}-\mathrm{NMR}$ spectra were recorded on a Mercury $300 \mathrm{MHz}$ NMR spectrometer in deuterium solvents to determine the composition of the polymer or to quantitatively monitor the monomer contents during the polymerization process. Glass transition temperatures were determined on a Perkin Elmer Pyris-1 differential scanning calorimeter under helium atmosphere. All the samples were first heated to $150^{\circ} \mathrm{C}$, then cooled to $-70^{\circ} \mathrm{C}$ and reheated to $150^{\circ} \mathrm{C}$ at the rate of $40^{\circ} \mathrm{C} / \mathrm{min}$. Thermogravimetric analysis (TGA) was performed on a Mettler Toledo TGA/SDTA851 instrument. All the samples were heated from $25^{\circ} \mathrm{C}$ to $500^{\circ} \mathrm{C}$ at the rate of $20^{\circ} \mathrm{C} / \mathrm{min}$ in air atmosphere.

The refractive index increment, dn/dc, of the copolymers was measured on a Waters 410 Differential Refractometer, which was calibrated by using $\mathrm{NaCl}$ aqueous solutions. The 
molecular weight of the polymer was determined on a GPC-MALLS light scattering system with a Waters GLC/GPC 244 apparatus in combination with a Dawn DSP MALLS light scattering photometer (Wyatt Technology Co.). Dimethylformamide (DMF) with a flow rate of $0.8 \mathrm{ml} / \mathrm{min}$ was used as mobile phase at room temperature.

\section{$\underline{\text { Results and Discussion }}$}

\section{Polymerization}

Copolymers synthesized from solution polymerization are listed in Table 1. The polymerization process could be monitored by Raman spectroscopy. Figure 1 shows the Raman spectra collected in the polymerization process of synthesizing copolymer P10. Figure 1a was the spectrum of the reaction mixture at the beginning of the polymerization. The bands at 3108 and $3040 \mathrm{~cm}^{-1}$ are the stretching modes of unsaturated $=\mathrm{C}-\mathrm{H}$ of the monomers. The strong band at $1638 \mathrm{~cm}^{-1}$ and the band at 1412 $\mathrm{cm}^{-1}$ are due to the stretching mode and in-plane bending of the alkene $\mathrm{C}=\mathrm{C}$ of the monomers, respectively. The band at $1714 \mathrm{~cm}^{-1}$ is the carboxylate carbonyl stretching mode. The wide bands at 2942, 2877, 2835, 1453, and $1299 \mathrm{~cm}^{-1}$ are the symmetric or asymmetric stretching or bending modes of $\mathrm{CH}_{3}$ or $\mathrm{CH}_{2}$ of the monomers and methanol. When the polymerization mixture was kept at $65^{\circ} \mathrm{C}$ for $30 \mathrm{~min}$, all the bands of the alkene $\mathrm{C}=\mathrm{C}$ at $3108,3040,1638$, and $1413 \mathrm{~cm}^{-1}$ have diminished as shown in Figure $1 \mathrm{~b}$. After the polymerization was kept for four hours, all the bands of the alkene $\mathrm{C}=\mathrm{C}$ disappeared (see Figure 1c). During the polymerization process, the frequency of the carbonyl band shifted from $1714 \mathrm{~cm}^{-1}$ (monomers) to $1728 \mathrm{~cm}^{-1}$ (polymers). When monomers polymerized to form polymers, the unsaturated $\mathrm{C}=\mathrm{C}$ bonds of monomers were converted to saturated C-C bonds, and the carboxylate carbonyl was no longer conjugated with $\mathrm{C}=\mathrm{C}$, thus the band shift to a higher frequency. With the aid of the FT-Raman spectrum, the progress of the polymerization can be conveniently monitored. However, it was also found that the sensitivity of FT-Raman is not high enough to quantitatively monitor the polymerization process. It was also difficult to distinguish the BA and 
MAPTAC with FT-Raman. Therefore, the copolymerization kinetics of this reaction was studied using NMR, as described later.

\section{Molecular Weight}

The molecular weights of the copolymers were determined with a GPC-MALLS light scattering system and are listed in Table 1 . Comparing the molecular weights of copolymers synthesized in different solvents, it is obvious that the molecular weights of the copolymers synthesized in methanol were much higher than those synthesized in ethanol. This can be ascribed to the chain transfer constant for ethanol being much larger than that for methanol. Nandi ${ }^{16}$ has reported that, in the radical polymerization of butyl acrylate at $80{ }^{\circ} \mathrm{C}$, the chain transfer constant for methanol was $4.7 \times 10^{-5}$ and for ethanol was $4.28 \times 10^{-4}$. The chain transfer constant for ethanol and methanol from MAPTAC radicals has not been reported, but the same trend as that from butyl acrylate radicals would be expected. It is also possible that the difference in solubility of the copolymer in the two solvents may affect the molecular weight. Since homogeneous copolymerization was observed in the synthesis of all the copolymers, this effect may be less important. Introducing a small amount of crosslinker EGDM significantly increased the molecular weight of the copolymer, but gelation occurred when crosslinker content was high.

\section{Compositions}

The composition of the resulting copolymer was determined from ${ }^{1} \mathrm{H}-\mathrm{NMR}$. A typical ${ }^{1} \mathrm{H}-\mathrm{NMR}$ spectrum of BA/MAPTAC copolymer in $\mathrm{CDCl}_{3}$ is shown in Figure 2. Chemical shifts of $4.0 \mathrm{ppm}$ and $3.40 \mathrm{ppm}$ were assigned to the $\mathrm{OCH}_{2}$ of $\mathrm{BA}$ and $\mathrm{N}^{+}\left(\mathrm{CH}_{3}\right)$ of MAPTAC, respectively. The composition of the copolymer was calculated from the integration ratio of the two peaks. The feed ratios of various monomer mixtures, as well as the composition of the resulting copolymers, were summarized in Table 1. It can be seen that the molar fractions of butyl acylate and cationic comonomer in the copolymer were all close to the feed ratios. 
The GPC chromatogram indicated that there was only one major peak for every copolymer. In an emulsion copolymerization of styrene and MAPTAC, van Streun and his coworkers [9] found that two fractions of copolymers (a high MAPTAC containing and a high styrene containing fractions) were formed when the cationic monomer feeding ratio is high. They indicated that the formation of two fractions was due to the significant difference in the solubility of two monomers. However, because the solvents used in this study are good solvents for both monomers of BA and MAPTAC and the copolymers, homogeneous copolymers are expected. The single peak of GPC supports that no homopolymers were presented in these systems. In order to further verify that the resulting polymers were uniform copolymers, some polymers were dissolved in chloroform and extracted with water. The compositions of the two fractions in water (a good solvent for polyMAPTAC) and chloroform (a good solvent for polybutyl acrylate) were measured by ${ }^{1} \mathrm{H}-\mathrm{NMR}$, and no apparent composition difference was found from the copolymers obtained from these two fractions. Therefore, it was concluded that the resulting copolymers are homogeneous copolymers.

\section{Copolymerizability}

The polymerization process was monitored quantitatively by NMR in $\mathrm{CD}_{3} \mathrm{OD}$, in which both the monomers and the resulting polymers were soluble. Figure 3a shows the NMR spectrum of a BA and MAPTAC mixture in methanol before initiator was added. Figure 3b shows the NMR spectrum of the above mixture after 10 minutes of polymerization. Conversion of BA was determined by change of the signal integration ratio of $\mathrm{OCH}_{2}$ protons (from $\delta 4.16 \mathrm{ppm}$ for monomer to $4.08 \mathrm{ppm}$ for polymer), and conversion of MAPTAC was determined by change of the signal integration ratio of $\mathrm{N}^{+}\left(\mathrm{CH}_{3}\right)_{3}$ protons (from $\delta 3.14 \mathrm{ppm}$ for monomer to $3.22 \mathrm{ppm}$ for polymer). The copolymer composition was calculated from the signal integration ratio of $\mathrm{OCH}_{2}$ protons and $\mathrm{N}^{+}\left(\mathrm{CH}_{3}\right)_{3}$ protons in polymer.

Figure 4 shows the time-conversion curves for the copolymerization of BA with MAPTAC in ethanol (Sample P3). It shows that the reactivity rate of MAPTAC is much 
higher than that of BA. To further understand the copolymerizability of BA and MAPTAC, their reactivity ratios were measured. Monomer reactivity ratios were evaluated by the graphical method according to the Fineman and Ross equation [16]:

$$
\frac{F(f-1)}{f}=r_{1} \frac{F^{2}}{f}-r_{2}
$$

where $r_{1}$ and $r_{2}$ are the reactivity ratios relating to BA $\left(M_{1}\right)$ and MAPTAC $\left(M_{2}\right)$, respectively; $F=d\left[M_{1}\right] / d\left[M_{2}\right]$ is the ratio of the numbers of each kind of repeat unit in the polymer; and $\mathrm{f}=\left[\mathrm{M}_{1}\right] /\left[\mathrm{M}_{2}\right]$ is the monomer molar feed ratio. Monomer reactivity ratios were also obtained by using the Kelen-Tudos method [17], which is a refined linearization method from the Fineman and Ross method.

Details of the copolymerization of BA with MAPTAC in ethanol and methanol are listed in Tables 2 and 3, respectively. The monomer reactivity ratios calculated from both the Fineman-Ross method and the Kelen-Tudos method are shown in Table 4. It is interesting to note that the solvents (ethanol and methanol) did not have a big effect on the relative copolymerizability of BA, but had a significant effect on that of MAPTAC. The high MAPTAC reactivity ratio in ethanol may be due to a "microphase separation effect" [18]. It is known that MAPTAC is more soluble in methanol than in ethanol [19]. The tendency for MAPTAC monomers to form aggregates in ethanol would be higher, and thus it would be more favorable for MAPTAC to have homopolymerization in ethanol than in methanol. To further understand the blockiness of the copolymer, the statistical distribution of monomer sequences $\mathrm{M}_{1}-\mathrm{M}_{1}, \mathrm{M}_{2}-\mathrm{M}_{2}$, and $\mathrm{M}_{1}-\mathrm{M}_{2}$ in the BA/MAPTAC copolymers was calculated by the method of Igarashi [20]. Table 5 lists the structural data for the copolymers. The calculated mol\% of $\mathrm{M}_{1}-\mathrm{M}_{2}$ linkages is much higher than that of $\mathrm{M}_{2}-\mathrm{M}_{2}$ linkages for all the copolymers, indicating that the blockiness of the MAPTAC is low even for copolymers prepared in ethanol. The low blockiness of MAPTAC is further indicated by the low mean sequence length value for MAPTAC in the copolymer. The reasons for this are the low MAPTAC feed ratio in the copolymerization and BA's preference for copolymerization. The results indicate that the difference of the monomer sequence distribution for the copolymers prepared in ethanol and methanol with a low MAPTAC content is not significant. 
Thermal Analysis

The glass transition temperatures $\left(\mathrm{T}_{\mathrm{g}}\right)$ of the PSA samples were determined by DSC, and the results are shown in Figure 5. It is obvious that with increased cationic content, the $\mathrm{T}_{\mathrm{g}}$ of the copolymer increases. Copolymers synthesized in methanol showed slightly higher $T_{g}$, which may be due to the higher molecular weight of the copolymer. Generally, copolymers from different solvents with similar cationic content showed very similar $\mathrm{T}_{\mathrm{g}}$.

Thermogravimetric analyses (TGA) of the copolymers were also conducted. Figure 6 shows the TGA curves of copolymers P1-P4. It clearly indicates that all copolymers begin to decompose at $230^{\circ} \mathrm{C}$ with three stages of weight loss. With increased cationic MAPTAC content, the first stage of weight loss increases correspondingly. This stage of weight loss may be attributed to the thermal instability of the MAPTAC units in the copolymer. Copolymers synthesized in methanol showed similar TGA curves. Figure 7 shows the temperature at $10 \%$ weight loss of the copolymer as a function of the MAPTAC content in the copolymer. No obvious difference of thermal stability was found for copolymers with the same MAPTAC content but synthesized in different solvents.

\section{$\underline{\text { Conclusions }}$}

Homogeneous copolymers of hydrophobic BA and hydrophilic cationic MAPTAC can be synthesized by solution copolymerization in ethanol or methanol. The polymerization process was monitored by FT-Raman and NMR. The molecular weights of the copolymers were measured by GPC and light scattering. It was found that the copolymer prepared in methanol has much higher molecular weight than that prepared in ethanol, and the molecular weight of the copolymer could also be effectively increased by incorporating a small amount of crosslinker. 
Copolymerization of BA with MAPTAC in ethanol and methanol yields very different values of reactivity ratio for MAPTAC. The high reactivity ratio of MAPTAC in ethanol may be due to the microphase separation effect.

Thermal analyses of the copolymers indicate that with increased cationic monomer content, the $\mathrm{T}_{\mathrm{g}}$ of the copolymer increases, and the thermal stability decreases. Thermal analysis results further showed that copolymers from different solvents with the same MAPTAC content had very similar glass transition temperatures $\left(T_{g}\right)$ and thermal stability.

Table 1. Characteristics of Copolymers

\begin{tabular}{|c|c|c|c|c|c|c|c|}
\hline \multirow[t]{2}{*}{ Sample } & \multirow[t]{2}{*}{ Solvent } & \multirow{2}{*}{$\begin{array}{l}\text { EGDM } \\
(\mathrm{mol} \%)\end{array}$} & \multicolumn{2}{|c|}{ Cationic unit fraction (mol\%) } & \multirow[t]{2}{*}{$\mathrm{dn} / \mathrm{dc}$} & \multirow{2}{*}{$\begin{array}{l}\mathrm{M}_{\mathrm{W}} / 10^{5} \\
(\mathrm{~g} / \mathrm{mol})\end{array}$} & \multirow[t]{2}{*}{$\mathrm{M}_{\mathrm{w}} / \mathrm{M}_{\mathrm{n}}$} \\
\hline & & & in feed & in polymer & & & \\
\hline P1 & ethanol & 0 & 5.0 & 5.7 & 0.045 & 2.3 & 1.5 \\
\hline $\mathrm{P} 2$ & ethanol & 0 & 10.0 & 10.6 & .051 & 1.8 & 1.3 \\
\hline P3 & ethanol & 0 & 15.0 & 15.2 & 0.063 & 1.4 & 1.3 \\
\hline $\mathrm{P} 4$ & ethanol & 0 & 20.0 & 20.9 & 0.066 & 2.3 & 1.3 \\
\hline P5 & ethanol & 0.2 & 15.0 & 17.6 & 0.063 & 8.6 & 4.9 \\
\hline P6 & ethanol & 0.5 & 15.0 & 17.3 & 0.063 & 13 & 2.0 \\
\hline $\mathrm{P} 7 *$ & ethanol & 0.8 & 15.0 & - & - & - & - \\
\hline P8 & methanol & 0 & 5.0 & 5.7 & 0.045 & 6.3 & 3.0 \\
\hline P9 & methanol & 0 & 10.0 & 10.8 & 0.051 & 7.8 & 4.1 \\
\hline P10 & methanol & 0 & 15.0 & 16.2 & 0.063 & 8.3 & 3.5 \\
\hline P11 & methanol & 0 & 20.0 & 21.9 & 0.066 & 9.7 & 4.2 \\
\hline P12 & methanol & 0.1 & 15.0 & 17.4 & 0.057 & 19 & 1.6 \\
\hline P13* & methanol & 0.2 & 15.0 & - & - & - & - \\
\hline
\end{tabular}

* Gel was formed during copolymerization. 
Table 2. Copolymerization of BA $\left(\mathrm{M}_{1}\right)$ with MAPTAC $\left(\mathrm{M}_{2}\right)$ in Ethanol

\begin{tabular}{|c|c|c|c|c|}
\hline \multirow{2}{*}{$f=\frac{\left[M_{1}\right]}{\left[M_{2}\right]}$} & \multirow{2}{*}{$\begin{array}{l}\text { Time } \\
\text { (min) }\end{array}$} & \multicolumn{2}{|c|}{ Conversion (\%) } & \multirow{2}{*}{$F=\frac{d\left[M_{1}\right]}{d\left[M_{2}\right]}$} \\
\hline & & $\mathrm{M}_{1}$ & $\mathrm{M}_{2}$ & \\
\hline 7.71 & 4 & 9.8 & 12.7 & 5.96 \\
\hline 4.87 & 4 & 9.2 & 12.2 & 3.69 \\
\hline 3.52 & 5 & 8.1 & 11.7 & 2.44 \\
\hline 2.53 & 5 & 7.0 & 11.2 & 1.59 \\
\hline 1.52 & 5 & 5.2 & 8.9 & 0.89 \\
\hline
\end{tabular}

Table 3. Copolymerization of BA $\left(\mathrm{M}_{1}\right)$ with MAPTAC $\left(\mathrm{M}_{2}\right)$ in Methanol

\begin{tabular}{lllll}
\hline$f=\frac{\left[M_{1}\right]}{\left[M_{2}\right]}$ & Time & \multicolumn{2}{l}{ Conversion (\%) } & $F=\frac{d\left[M_{1}\right]}{d\left[M_{2}\right]}$ \\
\cline { 3 - 4 } & $(\mathrm{min})$ & $\mathrm{M}_{1}$ & $\mathrm{M}_{2}$ & 6.13 \\
\hline 7.44 & 4 & 9.1 & 11.9 & 3.92 \\
4.68 & 4 & 7.8 & 9.3 & 3.08 \\
3.58 & 5 & 7.9 & 9.2 & 2.13 \\
2.52 & 5 & 7.0 & 8.3 & 1.39 \\
1.52 & 5 & 5.2 & 5.7 & \\
\hline
\end{tabular}

Table 4. Monomer reactivity ratios.

\begin{tabular}{llllllll}
\hline $\mathrm{M}_{1}$ & $\mathrm{M}_{2}$ & Solvent & \multicolumn{2}{c}{ Fineman-Ross Method } & & \multicolumn{2}{c}{ Kelen-Tudos Method } \\
& & & $\mathrm{r}_{1}$ & $\mathrm{r}_{2}$ & & $\mathrm{r}_{1}$ & $\mathrm{r}_{2}$ \\
\hline BA & MAPTAC & Ethanol & 0.91 & 2.56 & & 0.92 & 2.61 \\
BA & MAPTAC & Methanol & 0.79 & 0.93 & & 0.79 & 0.90 \\
\hline
\end{tabular}


Table 5. Structural data for the copolymers of BA $\left(\mathrm{M}_{1}\right)$ with MAPTAC $\left(\mathrm{M}_{2}\right)$

\begin{tabular}{|c|c|c|c|c|c|c|}
\hline \multirow[t]{2}{*}{ Copolymer } & \multirow{2}{*}{$\begin{array}{l}\mathrm{M}_{1} \text { in } \\
\text { copolymer } \\
(\mathrm{mol} \%)\end{array}$} & \multicolumn{2}{|c|}{ Blockiness (mol\%) } & \multirow{2}{*}{$\begin{array}{l}\begin{array}{l}\text { Alteration } \\
\text { (mol\%) }\end{array} \\
\mathrm{M}_{1}-\mathrm{M}_{2}\end{array}$} & \multicolumn{2}{|c|}{ Mean seq. length } \\
\hline & & $\mathrm{M}_{1}-\mathrm{M}_{1}$ & $\mathrm{M}_{2}-\mathrm{M}_{2}$ & & $\mathrm{M}_{1}$ & $\mathrm{M}_{2}$ \\
\hline P1 & 94.3 & 89.3 & 0.70 & 0.10 & 18.9 & 1.14 \\
\hline P2 & 89.4 & 81.0 & 2.18 & 16.8 & 10.6 & 1.26 \\
\hline P3 & 84.8 & 73.7 & 4.23 & 22.1 & 7.66 & 1.37 \\
\hline P4 & 79.1 & 65.4 & 7.17 & 27.4 & 5.76 & 1.52 \\
\hline P8 & 94.3 & 88.8 & 2.34 & 10.9 & 17.3 & 1.04 \\
\hline P9 & 89.2 & 79.3 & 8.82 & 19.8 & 8.99 & 1.09 \\
\hline P10 & 83.8 & 69.6 & 2.05 & 28.3 & 5.92 & 1.14 \\
\hline P11 & 78.1 & 60.1 & 3.86 & 36.1 & 4.33 & 1.21 \\
\hline
\end{tabular}

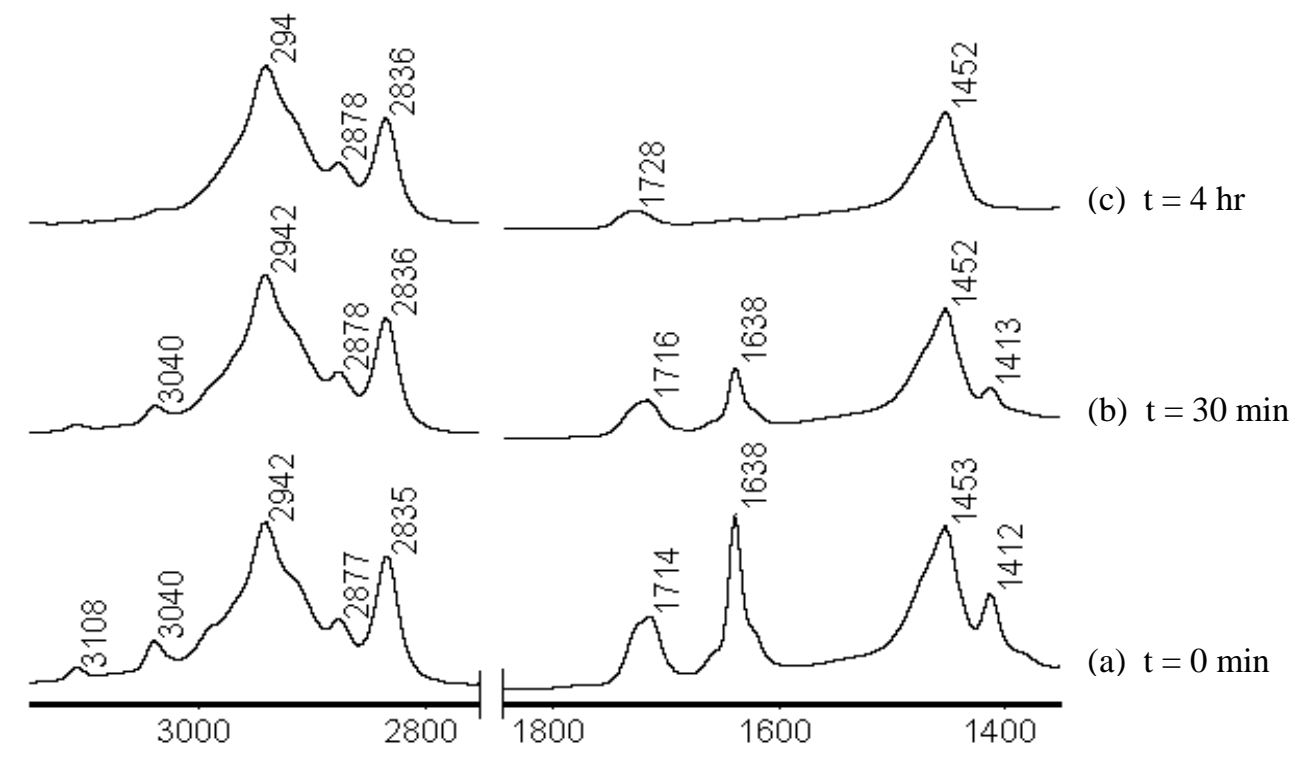

Figure 1. FT-Raman of the reaction mixtures at different times in the synthesis of BA/MAPTAC copolymer (P10): (a) 0 minute; (b) 30 minutes; (c) 4 hours. 

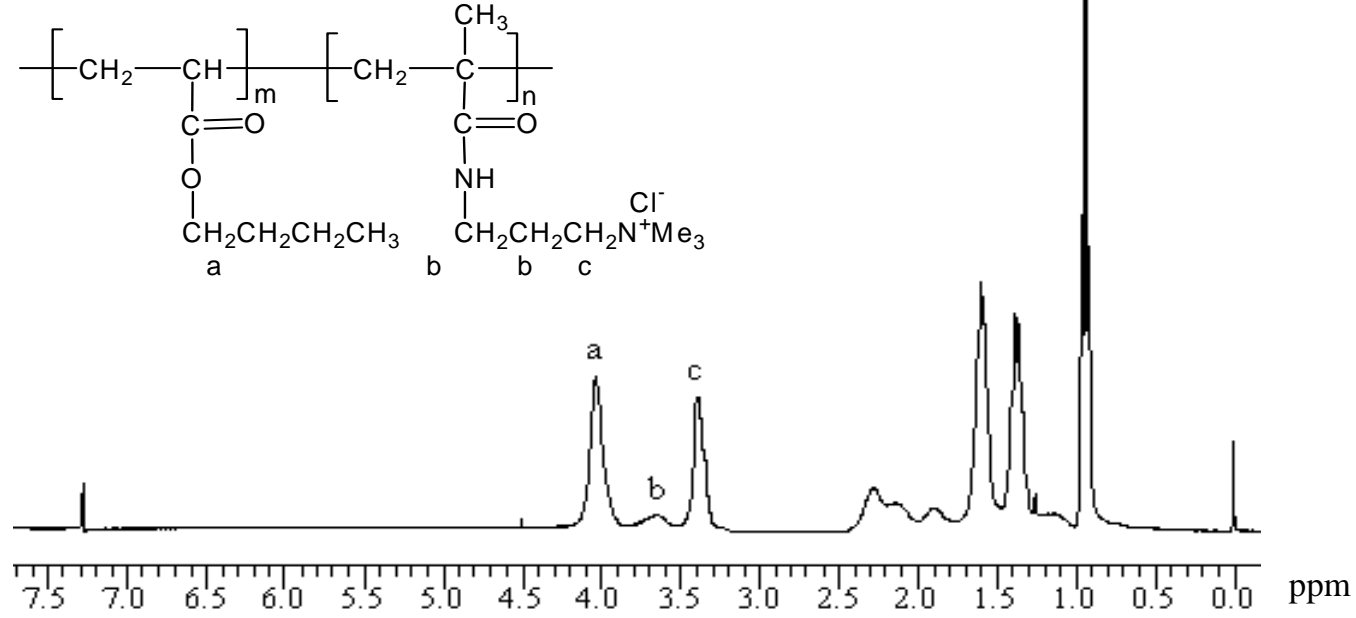

Figure 2. ${ }^{1} \mathrm{H}-\mathrm{NMR}$ spectrum of BA/MAPTAC copolymer (P3) in $\mathrm{CDCl}_{3}$.

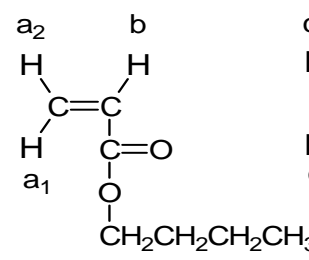

d

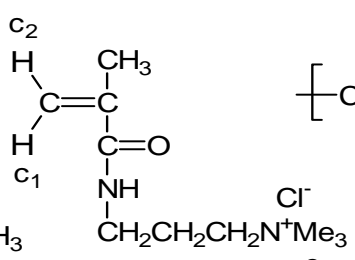

e

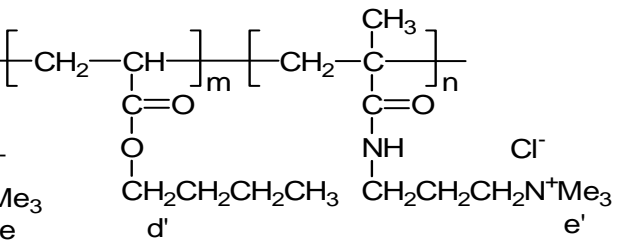

(b) $\mathrm{t}=10 \mathrm{~min}$

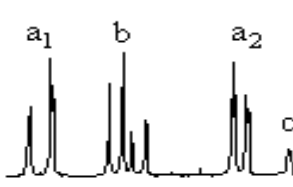

$c_{1}$ $\mathrm{c}_{2}$ $a_{2}$

(a) $\mathrm{t}=0$ min
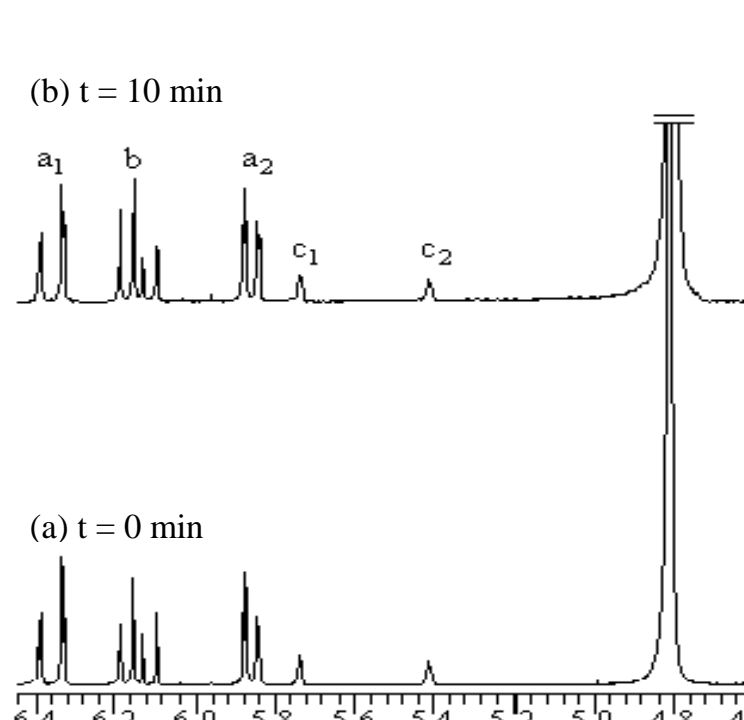


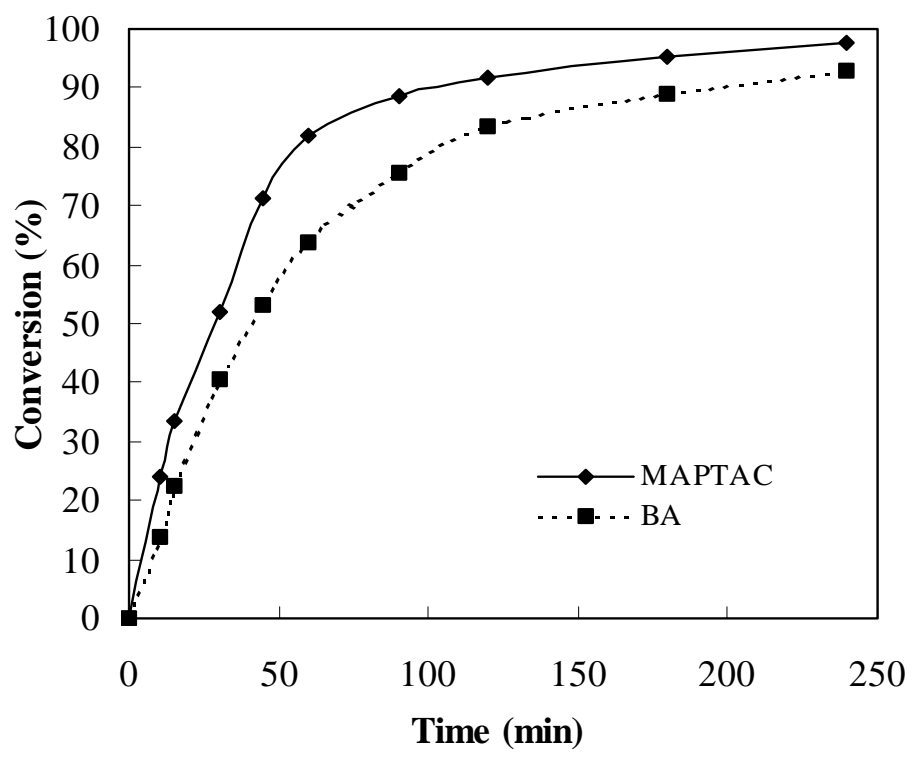

Figure 4. Time-conversion curves for the copolymerization of BA with MAPTAC in ethanol (P3).

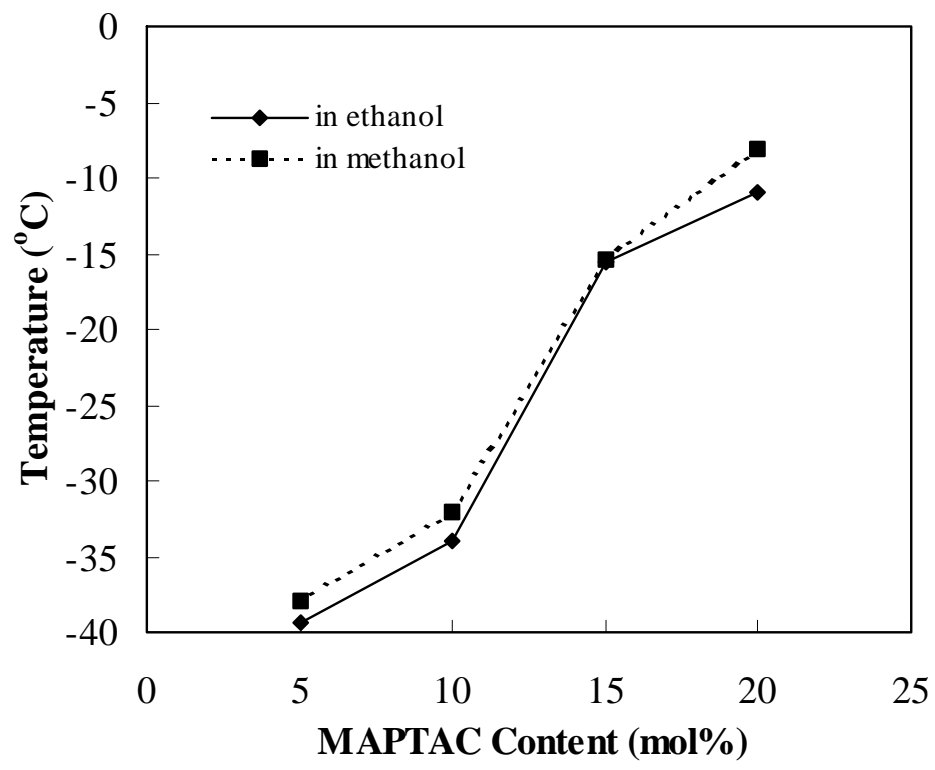

Figure 5. Glass transition temperature (Tg) of BA/MAPTAC copolymers as a function of the MAPTAC content in the copolymer. 


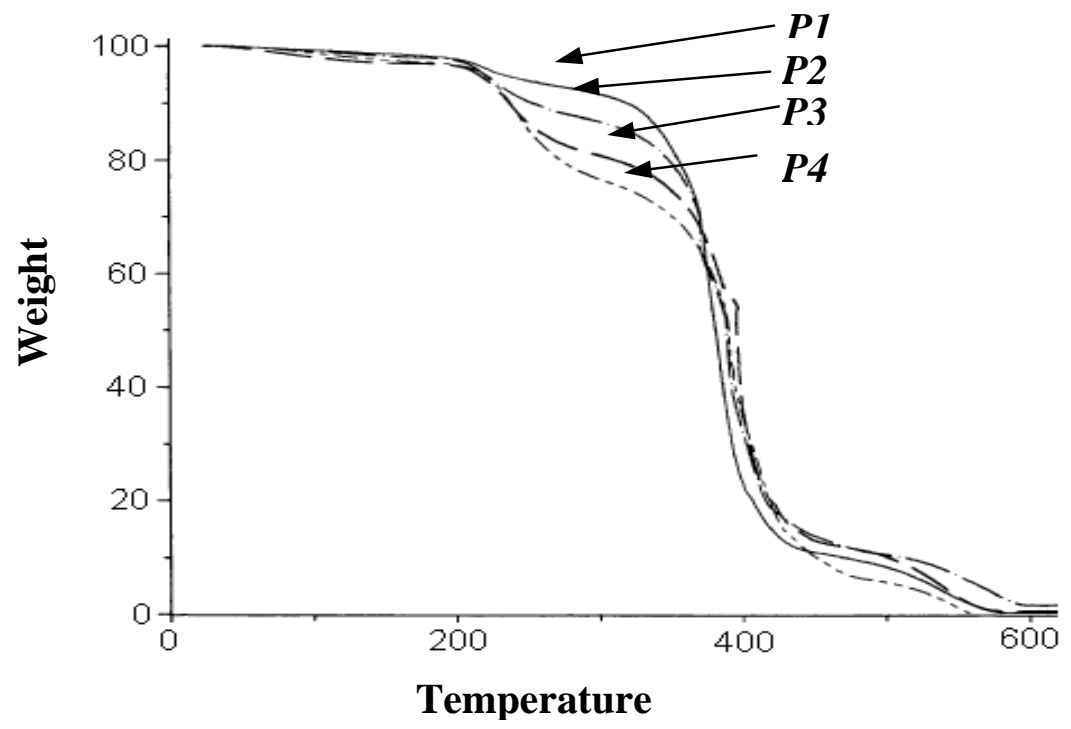

Figure 6. Thermogravimetric analysis (TGA) of BA/MAPTAC copolymers.

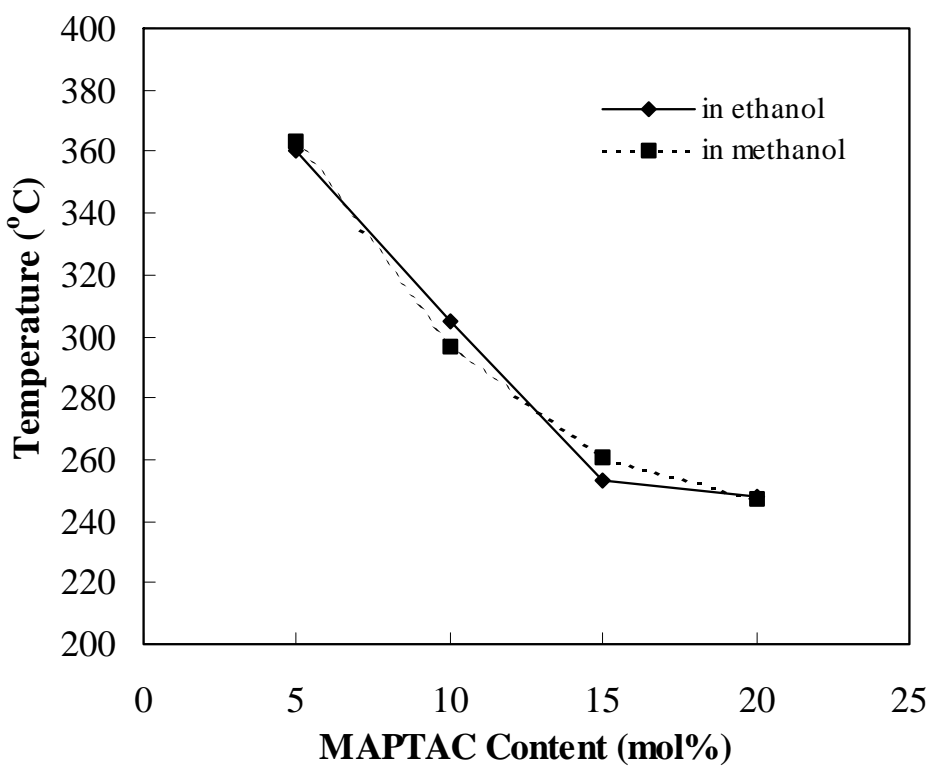

Figure 7. Thermogravimetric analysis of BA/MAPTAC copolymers: Temperature at $10 \%$ weight loss as a function of the MAPTAC content in the copolymer. 


\subsection{PSA Properties and Recyclability}

A range of cationic copolymers have already been synthesized from solvent polymerization as PSAs. We then studied their PSA properties and effect on paper recycling.

\section{Experimental}

\section{Solubility}

Solubility of the PSA in water was measured according to the following procedure: $0.4 \mathrm{~g}$ of dried PSA was added to $100 \mathrm{~mL}$ of water at $\mathrm{pH}$ 8.0. The mixture was stirred at $50^{\circ} \mathrm{C}$ for half an hour. The solubility was identified from the visual appearance of the solution. With the water-dispersible sample, the particle size of the dispersed particle was measure by Malvern Zeta-sizer.

\section{PSA Properties}

The $180^{\circ}$ peel strength was measured according to the Pressure Sensitive Tape Council standard method PSTC-1 (1994). The shear strength was measured according to the standard method PSTC-7 (1994).

\section{The Repulpability of PSA}

$50 \mu \mathrm{m}$ thickness of PSA was applied on a silicon release liner, dried at $90^{\circ} \mathrm{C}$ for $3 \mathrm{~min}$, and then transferred to commercial copy paper. The final PSA content was adjusted to $1 \%$ of the copy paper. The paper was torn to small pieces and soaked in water ( $\mathrm{pH} 8.0)$ with $1 \%$ consistency for 16 hours. The mixture was heated to $50^{\circ} \mathrm{C}$ and disintegrated for 20,000 revolutions in the standard disintegrator with the propeller operating at $3000 \mathrm{rpm}$ in the stock. A handsheet was made according to the Technical Association of Pulp and 
Paper Industry (TAPPI) standard method T 261 and dyed. The stickies particles on the handsheet were identified by image analysis.

\section{PSA Adsorption on Wood Fibers and Accumulation in Process Water}

The adsorption isotherm of the cationic PSA on wood fibers was measured as follows. Copy paper with various PSA content amounts (range from $0.5 \%$ to $8 \%$ based on oven-

dried paper) was repulped. After standing for 4 hours, the pulp stock was centrifuged at $3000 \mathrm{rpm}$ for half an hour. The content of PSA in the supernatant clear solution was measured by colloidal titration or UV spectroscopy.

To determine the PSA accumulation in papermaking process water, pulp stock of $1 \%$ consistency with $0.25 \%$ PSA based on oven-dried paper was made. After standing for one hour, the stock was filtered through a 200 -mesh screen. $50 \mathrm{~mL}$ of filtrate was collected for analysis, and the rest of the filtrate was used for the next pulping experiment. In every step, about 20 percent of fresh water is needed to compensate the water lost in the last step. Water from another pulping process without PSA was collected as reference. Then the PSA content in the filtrate was measured.

The Effect of PSA on Paper Properties

Handsheet paper was made according to Tappi Method T 261. The tensile strength of the paper was measured by Instron. The sizing effect (paper hydrophobicity) was evaluated by the Hercules Sizing Test (HST) method. For the sizing test, the handsheet paper was dried on a drum drier at $115-120^{\circ} \mathrm{C}$ for 4 min. The HST test was conducted using $1 \%$ formic acid ink solution, $80 \%$ reflectance.

$\underline{\text { Results and Discussion }}$

PSA Polymer Characteristics 
The break energy of the polymer depends on the polymer $\mathrm{T}_{\mathrm{g}}$ and molecular weight $(\mathrm{Mw})$. It has been demonstrated that the $\mathrm{T}_{\mathrm{g}}$, molecular weight, and their distribution as well as surface energy are important properties for PSAs [21,22]. Commercial solvent-based acrylic PSA normally has molecular weight $\left(\mathrm{M}_{\mathrm{w}}\right)$ at 100,000 [23], and $\mathrm{M}_{\mathrm{w}}$ distribution between 1.4-1.8. Sometimes, a small amount of crosslinker is introduced to the PSA polymer to increase the polymer internal strength, and thus the $\mathrm{M}_{\mathrm{w}}$ of the polymer will be significantly increased.

Table 1 shows the characteristics of the cationic PSA polymers prepared in this study. It can be seen that the molar fractions of BA and the cationic comonomer in the PSA copolymer were all close to the feed ratios. The molecular weight of the polymer is slightly higher than the commercial PSA. In the polymerization, ethanol as the solvent also acted as a chain transfer agent. No other transfer agent has been added to optimize the molecular weight in this study. Introducing a small amount of crosslinker EGDM significantly increased the molecular weight of the copolymer, but gelation occurred when crosslinker content was high. The molecular weight distribution of the cationic PSAs is in the optimized range. The glass transition temperatures $\left(T_{g}\right)$ of the PSA samples are shown in Figure 1. It is obvious that with increased cationic content, the $T_{g}$ of the copolymer increases. With cationic content less than $20 \%$, the $\mathrm{T}_{\mathrm{g}}$ of the PSA polymer is in the reasonable range for normal PSA applications. Because the polymerization kinetics and polymer characterization of these cationic polymers have been reported previously, only the adhesive properties and recycling ability of these new cationic PSAs will be the focus of this paper.

As indicated before, this study is to synthesize cationically charged and watersoluble/dispersible PSA to solve the stickies problem in paper recycling. Therefore, the solubility or dispersibility of these cationic PSAs must be studied. Table 1 shows the relationship between cationic content and PSA solubility in water. PSA with cationic monomer content at about 5\% was not soluble in water. With increased cationic monomer in the polymer, the polymer became water dispersible. PSA polymer became water soluble when the cationic monomer content was more than $15 \%$. Cationic 
monomer MAPTAC is hydrophilic, while butyl acrylate is hydrophobic. When the cationic monomer content in the polymer increases, the polymer becomes more hydrophilic. Thus the water solubility increases.

\section{Adhesive Properties}

The function of PSAs is to ensure instantaneous adhesion upon application of light pressure. PSAs must possess viscous properties in order to flow and to be able to dissipate energy during the adhesive bonding process and must also be elastic and be able to store bond rupture energy to provide strong bonding. The end-use properties, which are essential in characterizing the nature of PSAs, include tack, peel adhesion, and shear. Tack measures the adhesive's ability to adhere quickly, peel adhesion measures its ability to resist removal through peeling, and shear measures its ability to hold in position when shear force is applied. These PSA end-use properties depend on the nature and composition of the PSA polymer. The adhesive properties of cationic PSAs on polyester films were measured in this study and compared with those of commercial products. The results are shown in Table 2. From Table 2, it can be seen that different commercial products showed significantly different adhesive properties. The peel and shear strengths can vary widely depending on the PSA applications. Generally, peelable PSA has weak shear and peel strengths, while nonpeelable PSA has very strong peel strength. Cationic PSAs have different PSA properties depending on the PSA composition.

Figure 2 shows the effect of cationic monomer (MAPTAC) on the PSA properties. When cationic monomer content increases, $180^{\circ}$ peel strength decreases, and shear strength generally increases. Peel adhesion is the force required to remove a PSA-coated film from a specific test surface under standard conditions (specific angle and rate). The measurement of peel adhesion involves a bonding step and a debonding or peeling step. The efficiency of the bonding process is related to the adhesive's ability to exhibit viscous flow. In order to achieve peel adhesion, the bonding stage involves some dwell time. The debonding process involves a rapid deformation of the adhesive mass. Thus, the higher the peel strength, the higher the PSA's ability to resist bond deformation at high 
strain rates. Peel strength gives a measure of adhesive or cohesive strength, depending on the mode of failure [24]. Shear resistance is measured as a force to pull the PSA material parallel to the surface to which it was affixed with a definite pressure [25]. It measures the cohesion strength of the PSA. For PSA with low cationic monomer content, the $T_{g}$ is low; thus, it is more viscous and more easily forms a continuous layer on substrate for strong bonding. On the other hand, because the molecules can flow easily, the cohesive bonding would be easier to break. PSA1-3 all have cohesion failures in peel and shear. The results indicate they have stronger bonding strength on substrate than cohesion strength. With increased cationic monomer content, the cohesion strength increases, but the molecule flow ability reduces. Thus the shear strength increases, and peel strength decreases.

Introducing a small amount of crosslinker into the polymer can increase the polymer cohesion strength. Figure 3 shows the effect of crosslinker on the PSA properties. It can be seen that with increased crosslinker content, the shear strength increases. The effect of crosslinker on peel strength is not significant. Too much crosslinker can significantly limit the ability of polymer diffusion on a substrate and thus may reduce the peel strength.

Summarizing the data in Figures 1 to 3 and Tables 1 to 2, it can be concluded that cationically charged pressure-sensitive adhesives with different peel, shear, and tacky strengths can be prepared depending on the requirements of the end-use properties. By introducing cationic components into the polyacrylate backbone, the PSAs can be water soluble or dispersible depending on the cationic comonomer content, molecular weight, and crosslinking degree.

\section{PSA Repulpability}

Figure 4 shows the repulpability of the cationic PSAs during the paper recycling process. It can be seen that PSA formed large stickies particles when the cationic content was low. When the cationic content was high, no stickies particles were observed. The 
repulpability of PSA is obviously dependent on the PSA's water solubility/dispersibility. PSA1 is not water soluble. PSA3 could disperse in water and form colloidal particles. PSA4 to 7 are water soluble. Although PSA2 as a bulky polymer can only swell in water, when it formed a very thin PSA layer (less than $25 \mu \mathrm{m}$ ) on paper and then was recycled, the shear at repulping broke the PSA film to invisible microparticles.

\section{PSA Adsorption Isotherm on Wood Fiber}

When cationic PSA on recycled paper dissolved or dispersed during repulping, it became either cationic charged molecules or microparticles. Because fibers are negatively charged, cationic charged materials would be able to adsorb on fibers. The adsorption capability would be dependent on both the cationic PSA and the fiber. Figure 5 shows the adsorption isotherm of PSA4 on the pulp of copy paper. It can be seen that when the total amount of cationic PSA in the copy paper is less than $4 \%$, more than $90 \%$ of PSA will be adsorbed on the fiber. Adsorption equilibrium could not be reached even when the PSA content in the copy paper reached $8 \%$. In the paper recycling plant, the recycled paper normally contains more anionic materials than pure copy paper. Thus more cationic PSA should be able to be removed from the papermaking system.

\section{Accumulation of PSA in Pulping Water}

Due to environmental concerns and the desire to save costs, papermaking mills are totally or significantly closing the water loops. When water loops are closed and the papermaking process water is reused, the contaminants in water increase. Currently, commercial water-soluble/dispersible PSAs are all negatively charged. They cannot adsorb on negatively charged fibers and be removed from the water system. Thus they will accumulate in the papermaking water system. When there are sudden changes of temperature, $\mathrm{pH}$, or electrolyte in the water, the soluble PSA at high concentration may precipitate and cause stickies problems. In order to avoid the problems, the process water would have to be treated frequently to remove the PSA. 
It is known that cationic PSA can adsorb on fiber. But it is worthwhile to see if the cationic water-soluble PSA will accumulate in a closed papermaking system. The accumulation of cationic PSA in the closed pulping water system was studied, and the results are shown in Figure 6. In the study, copy paper with $0.25 \%$ of cationic PSA4 was repulped at $1 \%$ consistency. In 15 pulping cycles, the PSA concentration in the pulping water was almost constant at about $0.6 \mathrm{mg} / \mathrm{L}$. The PSA concentration in the pulping water would be $25 \mathrm{mg} / \mathrm{L}$ if there were no PSA adsorption on fiber. This suggests that over 97\% of PSAs were adsorbed on fiber, and the accumulation of cationic PSA in the pulping water is negligible.

\section{The Effect of PSA on Paper Properties}

Cationic PSA can adsorb on fiber and be removed with the fiber during papermaking. Since the fiber surface would be partially modified by the adsorbed cationic PSA, it is necessary to know if the adsorbed PSAs have any effect on final paper properties. Figure 7 shows the effect of PSA2, which formed water-dispersible microparticles during repulping, on the paper tensile strength. Figure 8 shows the effect of PSA4, which formed water-soluble molecules, on the paper tensile strength. It was found that both the water-dispersible and water-soluble PSAs did not have obvious effects on paper tensile strength. The effect of cationic PSAs on paper sizing was also studied. It was found that with $1 \%$ of PSA in paper, the HST value of the paper increased from 0 second to a few seconds for PSA2 and PSA4. This slight increase of HST should be negligible.

\section{Conclusions}

Cationically charged PSAs with various end-use properties were developed. These PSAs could be water dispersible or soluble in paper recycling depending on the charge densities of the PSAs and would not form stickies problems in pulping and papermaking processes. The dispersed/dissolved PSA could adsorb on negatively charged fibers and be removed from the papermaking system. They would not accumulate in the process water. The adsorbed PSAs on the fibers did not have obvious effects on final paper properties. 
Results indicate that the new cationic PSA is a possible solution for the stickies problems in paper recycling.

Table 1. Characteristics of PSA copolymers

\begin{tabular}{|c|c|c|c|c|c|c|}
\hline \multirow[t]{2}{*}{$\overline{\text { Sample }}$} & \multicolumn{2}{|c|}{ Cationic unit fraction (mol\%) } & \multirow{2}{*}{$\begin{array}{l}\text { EGDM } \\
(\mathrm{mol} \%)\end{array}$} & \multirow{2}{*}{$\begin{array}{l}M_{w} / 10^{5} \\
(\mathrm{~g} / \mathrm{mol})\end{array}$} & \multirow[t]{2}{*}{$\mathrm{M}_{\mathrm{w}} / \mathrm{M}_{\mathrm{n}}$} & \multirow{2}{*}{$\begin{array}{l}\text { Solubility } \\
\left(\mathrm{H}_{2} \mathrm{O}, 50^{\circ} \mathrm{C}\right)\end{array}$} \\
\hline & in feed & in polymer & & & & \\
\hline PSA1 & 5.0 & 5.7 & 0 & 2.3 & 1.5 & No \\
\hline PSA2 & 8.0 & 8.6 & 0 & 2.0 & 1.3 & Swollen \\
\hline PSA3 & 10.0 & 10.6 & 0 & 1.8 & 1.3 & $<1.5 \mu \mathrm{m}$ \\
\hline PSA4 & 15.0 & 15.2 & 0 & 1.4 & 1.3 & Soluble \\
\hline PSA5 & 20.0 & 20.9 & 0 & 2.3 & 1.6 & Soluble \\
\hline PSA6 & 15.0 & 17.6 & 0.2 & 8.6 & 1.6 & Soluble \\
\hline PSA7 & 15.0 & 17.3 & 0.5 & 13 & 2.0 & Soluble \\
\hline PSA8 & 15.0 & - & 0.8 & Gel forn & ned in pol & ymerization \\
\hline
\end{tabular}


Table 2. Adhesive properties of cationic PSAs and commercial PSAs

\begin{tabular}{|c|c|c|c|c|}
\hline \multirow[t]{2}{*}{ Sample } & \multicolumn{2}{|c|}{$\begin{array}{l}180^{\circ} \text { Peel Adhesion } \\
\text { (10 min Dwell) (PSTC-1) }\end{array}$} & \multicolumn{2}{|c|}{ Shear Strength (PTSC-7) } \\
\hline & g/in & Failure Type $^{(\mathrm{e})}$ & Hour & Failure Type \\
\hline PSA1 & 653.7 & $\mathrm{C}$ & 0.2 & $\mathrm{C}$ \\
\hline PSA2 & 586.3 & $\mathrm{C}$ & 0.3 & $\mathrm{C}$ \\
\hline PSA3 & 532.0 & $\mathrm{C}$ & 0.4 & $\mathrm{C}$ \\
\hline PSA4 & 155.6 & A & 1.0 & A \\
\hline PSA5 & 70.8 & $\mathrm{~A}$ & 2.8 & $A$ \\
\hline PSA6 & 249.0 & $\mathrm{~A}$ & 5.7 & $\mathrm{~A}$ \\
\hline PSA7 & 110.4 & $\mathrm{~A}$ & $>40 \mathrm{~h}$ & No failure \\
\hline $\operatorname{Com}^{(\mathrm{a})}$ & 266.0 & $\mathrm{C}$ & 3.9 & $\mathrm{C}$ \\
\hline Com2 $2^{(b)}$ & 509.4 & A & $>40$ & No failure \\
\hline $\mathrm{Com}^{(\mathrm{c})}$ & 7.9 & $\mathrm{~A}$ & Instant failure & $\mathrm{A}$ \\
\hline Com4 $4^{(\mathrm{d})}$ & 939.6 & A & 3.7 & $\mathrm{~A}$ \\
\hline
\end{tabular}

${ }^{\mathrm{a}}$ Polyacrylic-based PSA from Company 1. ${ }^{\mathrm{b}}$ Polyacrylic-based PSA from Company 2. ${ }^{\mathrm{c}}$ Peelable note. ${ }^{\mathrm{d}}$ Nonpeelable general postal label. ${ }^{\mathrm{e}}$ A: adhesion failure; C: cohesion failure.

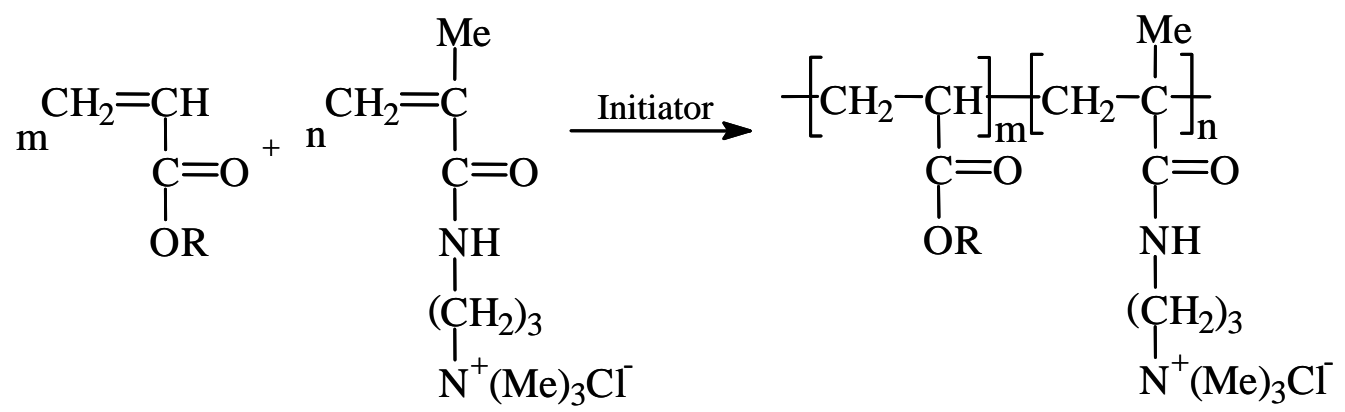

Scheme 1. Polymer synthesis. 


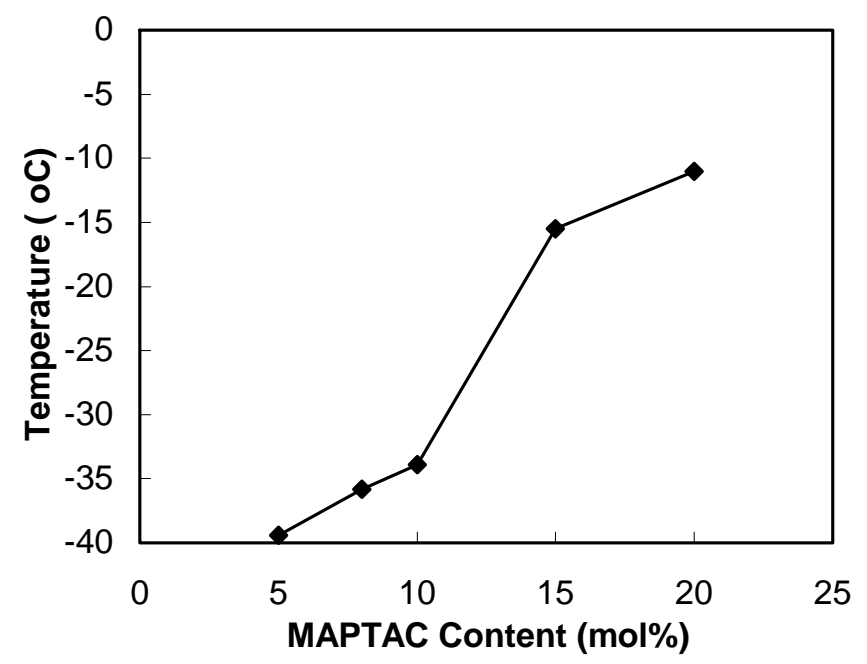

Figure 1. Glass transition temperature $\left(\mathrm{T}_{\mathrm{g}}\right)$ of BA/MAPTAC copolymers as a function of the MAPTAC content in the copolymer.

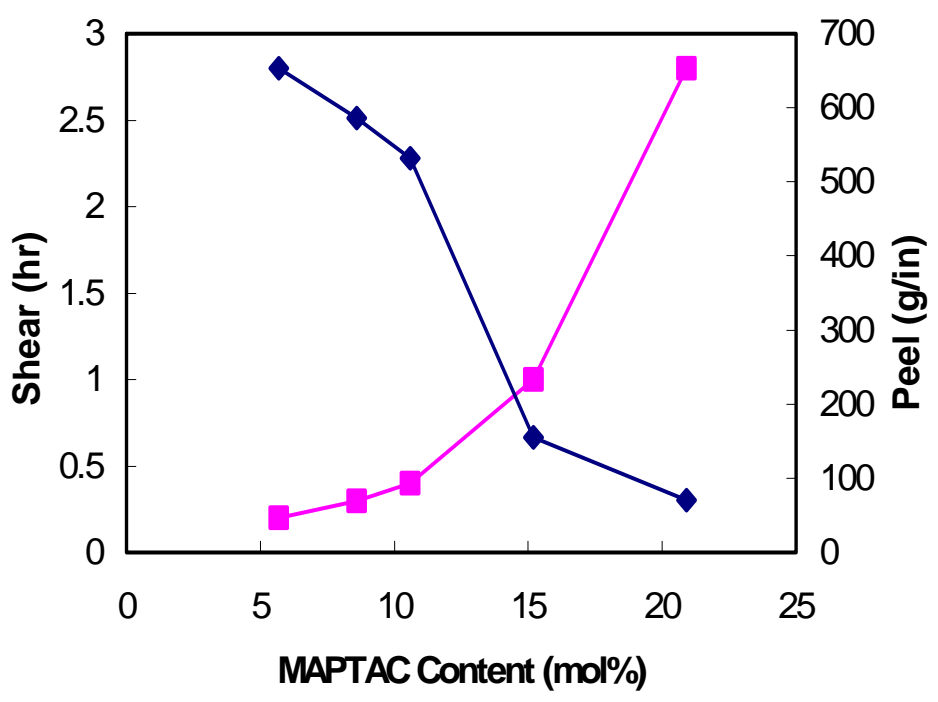

Figure 2. The effect of MAPTAC content in the PSA copolymer on the PSA properties. 


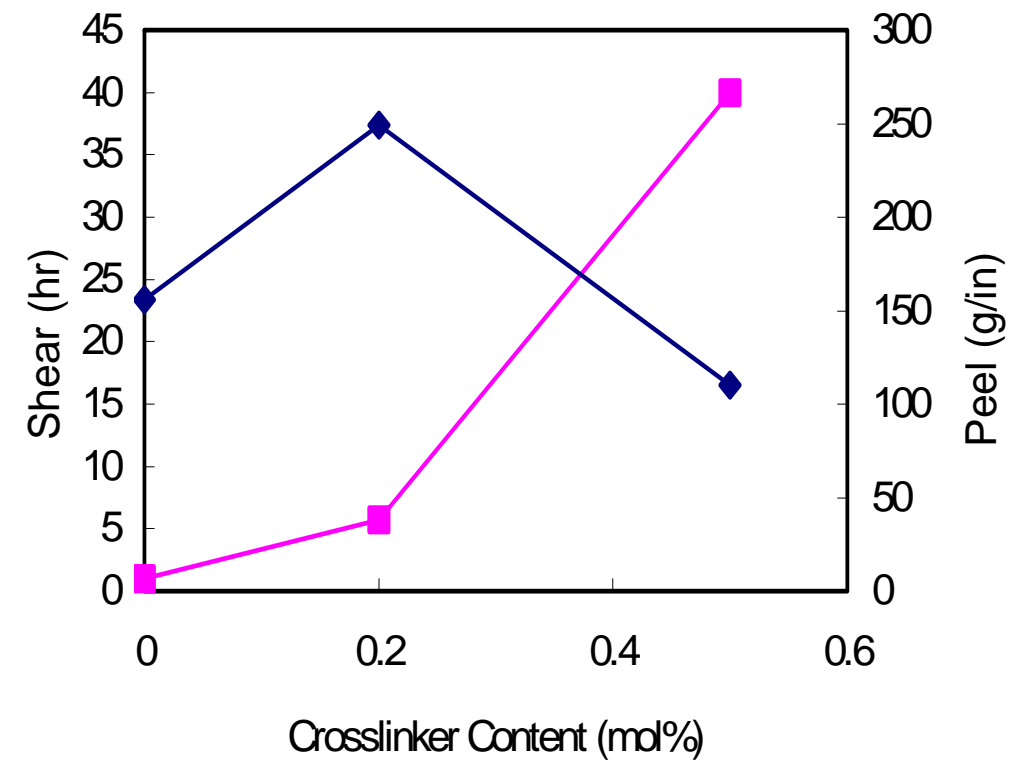

Figure 3. The effect of crosslinker content in the PSA copolymer on the PSA properties (PSA contains 15 mol\% MAPTAC).

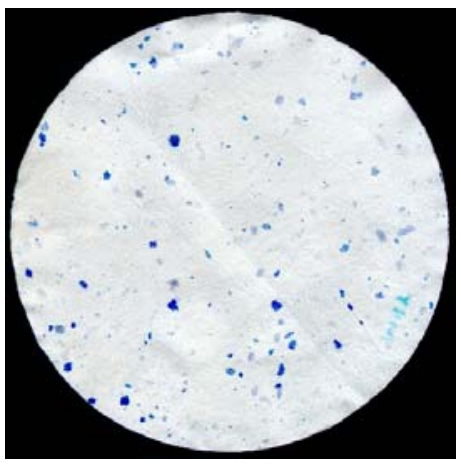

(A)

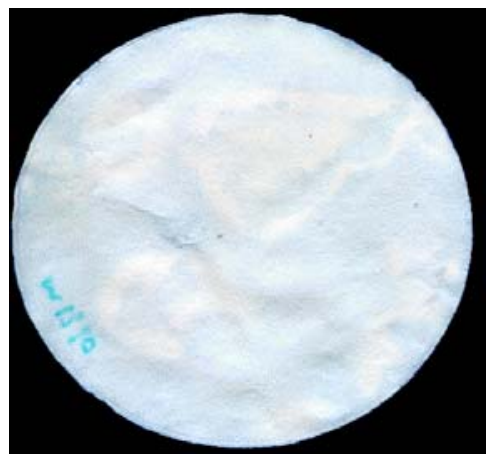

(B)

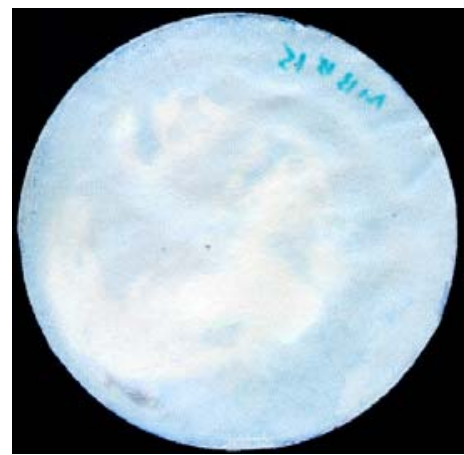

(C)

Figure 4. Repulpability of cationic PSAs. Cationic monomer content: (A) 5.7 mol\%; (B) $10.6 \mathrm{~mol} \%$; (C) $15.2 \mathrm{~mol} \%$. 


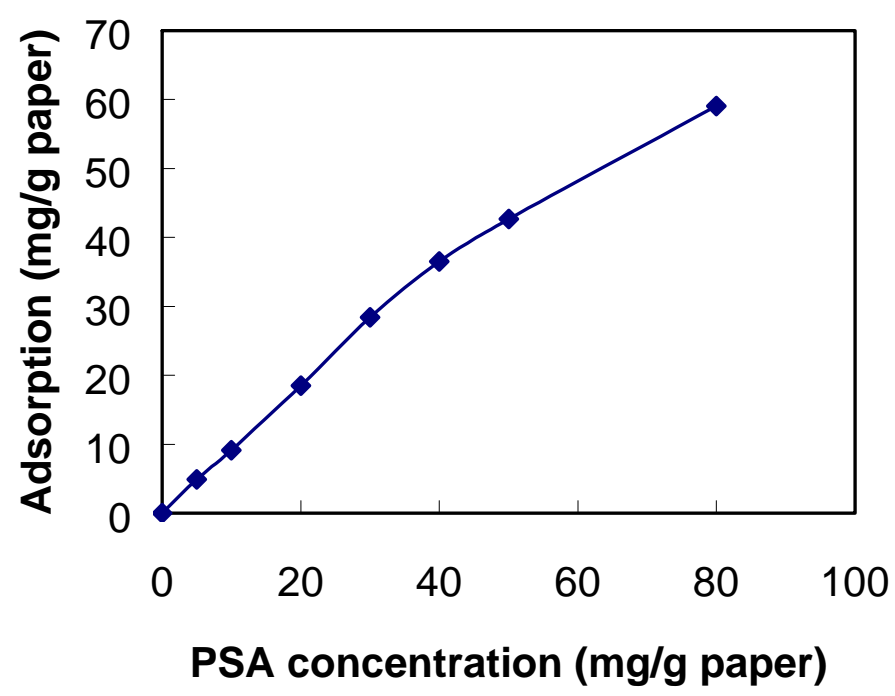

Figure 5. Adsorption isotherms of PSA4 on the wood fiber of office copy paper.

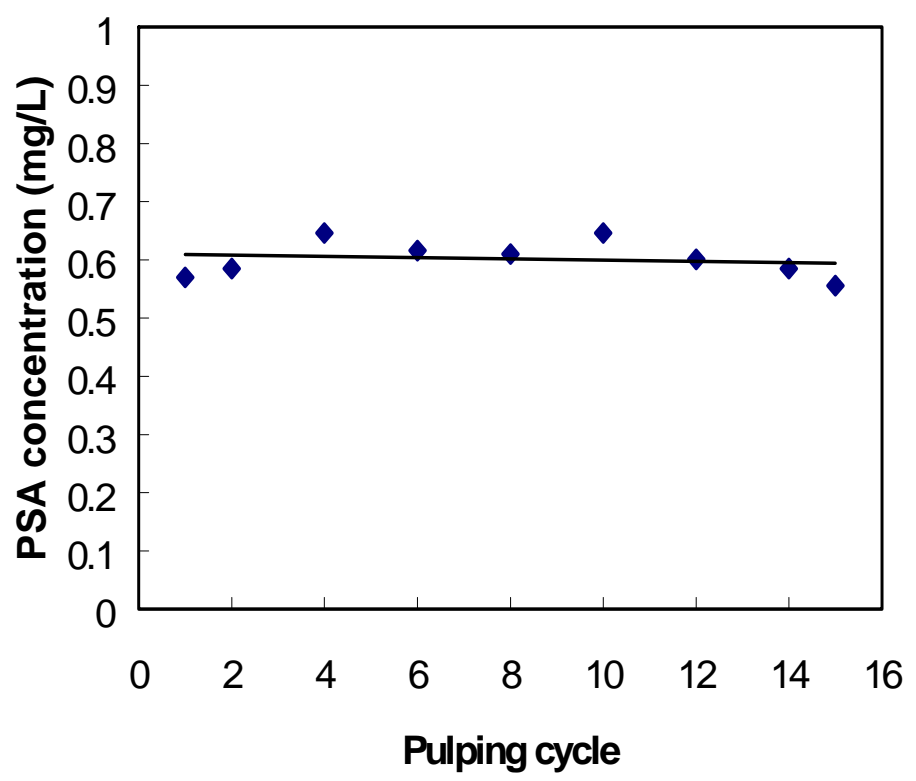

Figure 6. The accumulation of PSA4 in the closed pulping water system 


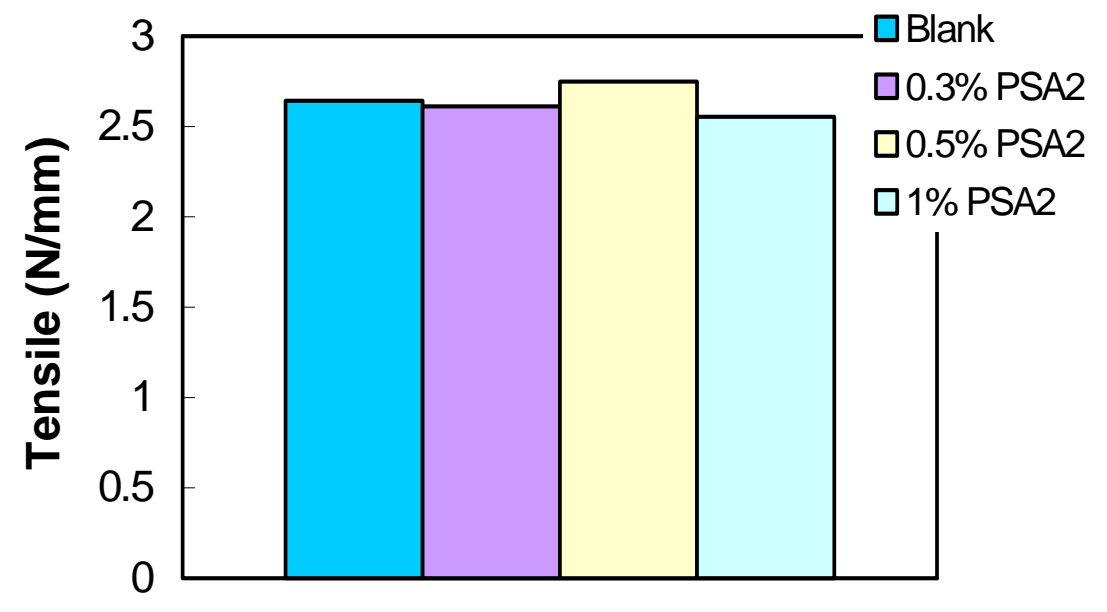

Figure 7. The effect of PSA2 on paper tensile strength.

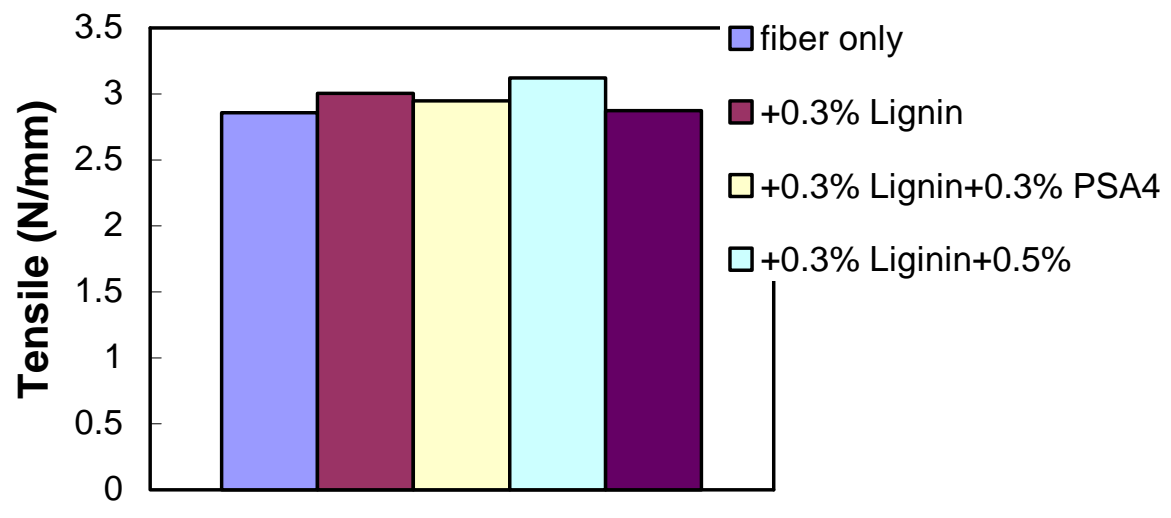

Figure 8. The effect of PSA4 on paper tensile strength 


\section{References}

1. M. Bernard, W.T. Ford and T.W. Taylor, Macromolecules, 17, 1812 (1984).

2. W.T. Ford and H. Yu, Langmuir, 9, 1999 (1999)..

3. B. Alince, Tappi J., 60(12), 133 (1977).

4. M. Inoue, B. Alince, Y. Shao and T.G.M. van de Ven, International Paper and Coating Chemistry Symposium, Ottawa, Canada, June 1996, pp 119.

5. H. Ono and Y. Deng, J. Colloid Interface Sci., 188, 183 (1997).

6. H. Ono and Y. Deng, Engineering and Papermakers Conference, Nashville, TN, 1997, pp 837.

7. O. Ishikawa, Y. Sasagawa, A. Tsuji and T. Yamashita, Jpn. Tappi J., 47, 334 (1993).

8. W.M. Brouwer, M. van Haeren and P. van Haeren, Eur. Polym. J., 26(1), 35 (1990).

9. K.H. van Streun, W.J. Belt, P. Piet and A.L. German, Eur. Polym. J., 27(9), 931 (1991).

10. D. Upson, J. Polym. Sci. Polym. Symp., 72, 45 (1985).

11. S. Bon, H. van Beek, P. Piet and A.L. German, J. Appl. Polym. Sci., 58, 19 (1995).

12. J. Otaigbe, R.E. Banks and S. Smith, Br. Polym. J., 20, 53 (1988).

13. Y. Deng, Z. Yan and N. Yang, Colloid. Polym. Sci., 277, 227 (1999).

14. N. Konar and C.J. Kim, J. Appl. Polym. Sci., 69, 263 (1998).

15. T. Nonaka, K. Fujita, J. Membr. Sci., 144 (1-2), 187 (1998).

16. M. Fineman and S.D. Ross, J. Polym. Sci., 5, 259 (1950).

17. T. Kelen and F. Tudos, J. Macromol. Sci. Chem., A9, 1 (1975).

18. A. Ledwith, G. Galli, E. Chiellini and R. Solaro, Polym. Bull., 1, 491 (1979).

19. W.-F. Lee and C.-C. Tsai, Polymer, 35, 2210 (1994).

20. S. Igarashi, J. Polym. Sci. Polym. Lett., 1, 359 (1963).

21. J. Kendall, F. Foley and S.G. Chu, Adhesive Age, 29(9), 26 (1986).

22. A. Midgley, Adhesive Age, 29(9), 17 (1986).

23. F.T. Sanderson, Adhesive Age, 26(12), 26 (1983).

24. P. Caton, European Adhesives and Sealants, 12, 18 (1990).

25. I. Benedek and L.J. Heymans, Pressure Sensitive Adhesive Technology, Marcel Dekker, Inc, New York, 214 (1997). 


\section{PART 2. WATER-SOLUBLE/DISPERSIBLE CATIONIC PRESSURE SENSITIVE ADHESIVES FROM EMULSION POLYMERIZATION}

\subsection{Summary}

As reported in Part 1, solvent based water soluble/dispersible and easily removable cationic PSAs have been successfully developed. We then developed water based cationic PSAs from emulsion polymerization. First, the macro- and miniemulsion polymerization of butyl acrylate (BA) with cumene hydroperoxide/tetraethlenepentamine (CHP/TEPA) as a redox initiator system was investigated. It was found that the rate of polymerization was monotonically decreasing rather than going through a maximum as is common in emulsion polymerization. Furthermore, the polymerization rate at high monomer conversion of macroemulsion polymerization was unexpectedly decreased with an increase in initiator concentration. For miniemulsion polymerization, the polymerization stopped at rather low conversion. It was also found that the average number of free radicals per particle dropped very quickly with polymerization time. With increased initiator concentration, the average number of radicals per particle decreased in the macroemulsion polymerization. Molecular weight analysis indicated that aqueous phase polymerization, interfacial polymerization and particle polymerization take place simultaneously. It is proposed that TEPA plays two roles in the polymerization; it is both a reducing agent in the redox initiator system, and a chain transfer agent. All the unexpected results are well explained by postulating this multiple function of TEPA and taking the heterogeneous nature of emulsion polymerization into account.

Then the polymerization kinetics of butyl acrylate/[2-(methacryloyoxy)ethyl]trimethyl ammonium chloride (BA/MAETAC) macroemulsion and miniemulsion copolymerization with CHP/TEPA as a redox initiator system was investigated. The postulate of interfacial copolymerization with the two-component redox initiator system (one hydrophobic and the other hydrophilic), was confirmed. Adding MAETAC had a complex effect on the polymerization kinetics of BA. The influence was ascribed to variations in the nucleation mechanism dependent on the level of MAETAC, and the polymerization method 
(macroemulsion versus miniemulsion). It is proposed that at the beginning of macroemulsion copolymerization with high MAETAC composition, micellar copolymerization occurs, which controls the nucleation process. Hydration properties of the latex were used to characterize the copolymer composition. The composition of the copolymer from the interfacial polymerization is very heterogeneous. With an increase in BA conversion or of the particle size, the copolymer composition is lower in BA. Adding salt increases MAETAC content and decreases BA content in the copolymer.

Finally, the end use properties of the PSAs were evaluated, and the reulpability of the PSAs in paper recycling was studied. It was found that the cationic PSA from miniemulsion polymerization itself was insoluble or non-dispersible in water during the paper recycling process. However, if this water-insoluble cationic PSA from miniemulsion was formulated with a water-soluble cationic PSA made from ethanol, the solubility or dispersibility of the former PSA in water was improved. The molecular weight and crosslinking degree of the PSA polymer have significant effects on the PSA properties and dispersability.

\subsection{Emulsion Polymerization of Butyl Acrylate with the Cumene Hydroperoxide/ Tetraethylenepentamine Redox Initiator}

Very recently, Gilbert [1] showed that the cumene hydroperoxide/tetraethlenepentamine (CHP/TEPA) redox initiator system is very useful in promoting grafting of acetate monomer onto the surface of natural rubber particles. CHP is hydrophobic and TEPA is hydrophilic, and hence the free radicals are formed at the particle-water interface, and this promotes surface grafting. Siadat [2] reported that, by the use of a nonionic surfactant and a two-component redox initiator (a water-soluble reductant and a hydrophobic peroxide), a copolymer with moderate ionic monomer content could be prepared. Turner [3] found, for a similar system, that the sulfonate content of the copolymer of a sodium styrene sulfonate/styrene emulsion copolymerization was dependent upon the initial sodium styrene sulfonate charge, but was always lower than the recipe amount. This behavior appeared not to be dependent on the type of initiator (water-soluble persulfate or 
a redox initiator with one component present in the hydrocarbon phase and the other in the aqueous phase). Their results contrast sharply with those of Siadat.

It is clear that kinetic studies of redox initiator systems such as CHP/TEPA, in which one component is water-soluble and the other is hydrophobic, are needed. As a preliminary to studies of emulsion copolymerization of butyl acrylate (BA) with cationic (strongly hydrophilic) monomers, the present work was undertaken to investigate the kinetics and mechanism of the emulsion polymerization of BA with the redox initiator CHP/TEPA.

\section{Experimental}

\section{Materials and Experimental Procedure}

All reagents were purchased from Aldrich Chemical. BA was purified by washing three times with a $10 \% \mathrm{NaOH}$ solution, followed by three washes with deionized water. Others reagents were used as received. Triton X-405 was used as surfactant. Hexadecane (HD) was used as cosurfactant in the miniemulsion polymerization. Table 1 shows the basic polymerization recipe.

A solution of de-ionized water and Triton X-405 was emulsified with a solution of BA and CHP (and HD in the cases of miniemulsion). To make a miniemulsion, sonication was then performed for 15 min with a $300 \mathrm{~W}$ sonic dismembrator at $70 \%$ power while a magnetic stirrer provided bulk mixing. For the macroemulsion (conventional emulsion) polymerizations no sonication was done. The macroemulsion or miniemulsion was added to the reactor and purged with nitrogen for at least 30 minutes to remove dissolved and vapor space oxygen. The reactor temperature was then raised from room temperature to $40{ }^{\circ} \mathrm{C}$ using a thermostated water bath. The polymerization was begun by injecting the recipe amount of TEPA solution. Samples were taken at intervals for analysis and stabilized with inhibitor. BA conversion was determined gravimetrically. 
The molecular weight distribution (MWD) of samples was determined by gel permeation chromatography (GPC) using a Waters 410 GPC with two columns: $5 \times 10^{5}$ and $5 \times 10^{2}$ $\AA$ The solvent was tetrahydrofuran (THF) and the flowrate was $1 \mathrm{ml} / \mathrm{min}$. Samples were inhibited, dried, then dissolved in THF for analysis. Particle size analysis was accomplished using a Protein Solution LSR-TC dynamic light scattering instrument. The latex was diluted with a Triton X-405 solution and kept in an oven at $80^{\circ} \mathrm{C}$ for 12 hours to drive off the unreacted BA prior to analysis.

\section{$\underline{\text { Results and Discussion }}$}

\section{Monomer Conversion}

The macroemulsion and miniemulsion polymerizations were carried out at various initiator concentrations. The conversion-time curves are shown in Figures 1 and 2. The polymerization rate is highest at the beginning of the reaction, and decays monotonically thereafter. For the macroemulsion polymerizations, the polymerization rate in the beginning increases with increase of initiator concentration. However, unexpectedly, the polymerization rate decays so much faster at higher initiator concentrations that the polymerization rate late in the reaction decreases with an increase of initiator concentration, even at the same conversion.

By contrast, the miniemulsion polymerizations kinetics look something like homogeneous polymerizations with a highly active initiator. At the beginning, the polymerization takes place rapidly. However, it decays very quickly so that the conversion-time curves level off at rather low conversions. It was found that if more TEPA was added to the system after the conversion-time curve has leveled off, the polymerization could be restarted, and proceeded quickly. It is clear from this that in miniemulsion polymerization, TEPA is consumed more rapidly than CHP, although the initiation mechanism indicates that CHP and TEPA should be consumed at equal rates. It would appear that some side reaction is consuming TEPA. 
For miniemulsion polymerization, the conversion-times curves have the same shape for both initiator concentrations, whereas the macroemulsion polymerization rate decays faster the higher the initiator concentration. In order to gain a better understanding, the average number of free radicals per particle was determined.

\section{Average Number of Free Radicals Per Particle}

The evolution of the number of particles with time is presented in Figures 3 and 4. It can be seen that the number of particles is high for macroemulsion polymerization, which indicates a high efficiency of nucleation. It is also found that limited aggregation takes place during the early stages of macroemulsion polymerization, and a distinct second nucleation occurred around a conversion of 40\%, which can be ascribed (per Ozdeger et al., 1997 in the comparable styrene system) to the partition behavior of Triton X-405 into the monomer droplets. In the late stage of macroemulsion polymerization, limited aggregation occurs once more, indicating a deficit of surfactant for colloidal stability. For miniemulsion polymerization, no limited aggregation and second nucleation are seen at the given monomer conversion. The lack of secondary nucleation in miniemulsion polymerization with nonionic surfactant is also reported by Schork [4]. It is interesting to note that, for miniemulsion polymerization, although nucleation ended earlier in terms of conversion, at lower initiator concentration, it ended at approximately the same time, regardless of initiator concentration (Figure 5).

From the particle number and conversion-time curves, the average number of free radical number per particle can be calculated as [5]:

$$
\bar{n}=\frac{R_{p} N_{a v} n_{M, 0}}{k_{p} C_{p} N_{p}}
$$

Here $R_{p}$ is the overall polymerization rate calculated as the slope of the conversion time curve in mol/s; $k_{\mathrm{p}}$ is the propagation rate constant, $\left[\mathrm{k}_{\mathrm{p}}=1.874 \mathrm{e} 11 * \exp (-7026.7 / \mathrm{T}), \mathrm{L} / \mathrm{mol}-\right.$ s] [6]. (Other values of $k_{p}$ might have been used; this value was used because the citation also gives a reference for the average radical number. For the present argument, the trend in $\bar{n}$ is more important than its absolute value.) $C_{p}$ is the monomer concentration in a 
particle. (For macroemulsion polymerization, the value at saturation [droplets present] was taken as $4.54 \mathrm{~mol} / \mathrm{L}$. After $40 \%$ conversion, the values were calculated as a function of conversion. For miniemulsion polymerization, the values were calculated as a function of conversion.) $\mathrm{N}_{\mathrm{p}}$ is the number of particles, calculated from the volume average particle size measured by dynamic light scattering. $\mathrm{N}_{\mathrm{av}}$ is Avogadro's constant. Finally, $\mathrm{n}_{\mathrm{M}, 0}$ is the initial number of moles of monomer per unit volume of water.

Figures 6 and 7 show $\bar{n}$ as a function of time. It has been reported that for BA macroemulsion polymerization, the average free radical number per particle $(\bar{n})$ may be up to $30[6,7]$. Here, it was found that $\bar{n}$ is much less than that of BA macroemulsion polymerization using more common initiators, and decreases with increase in initiator concentration. Clearly, exit of free radicals from the particles plays a significant role in the present system; this then implies significant chain transfer [5]. It has been reported that alkyl amine can function as a chain transfer agent in the macroemulsion polymerization [8], so it is plausible that TEPA should be a transfer agent in the present system. The assumption is in good agreement with the previous evidence that TEPA is being consumed by a side reaction, and will be supported by molecular weight distribution (MWD) data below. Thus, it is postulated that TEPA plays two roles in the system: reducing agent in the redox initiator system, and chain transfer agent. With increasing initiator concentration, the size of particles (inferred from the particle number data in Figure 3), decreases. Because the rate coefficient for escape of free radicals from the particle is proportional to the square of the radius of the particle, the exit rate of free radicals would be much increased with an increase of TEPA concentration. Additionally, because TEPA is an efficient chain transfer agent, the exit rate of free radicals would be increased. It is proposed that the enhancement in the exit rate by chain transfer to TEPA causes a decrease in $\bar{n}$ with increase an in initiator concentration.

Because of the difference in hydrophilicity between TEPA and CHP and monomer, it is proposed that most of the TEPA is consumed at the interface between the water phase and the particle by reaction with CHP or by chain transfer from a live polymer chain. The change in the size of the particle would exert a significant influence on TEPA reaction 
kinetics. With a decrease in particle size (increase in the total surface area), more TEPA would be reacted. That means that the TEPA concentration would decay more quickly. This is proposed as an explanation for why the polymerization rate in macroemulsion polymerization decays faster with the higher initiator concentration, as shown in Figure 1.

For miniemulsion polymerization, the particle size is more determined by the droplet size, so initiator concentration has little influence on the particle size. This is why, contrary to the effect seen in macroemulsions, the decay of the miniemulsion polymerization rate has little dependence on initiator concentration. Additionally, because most of the CHP is located in the particle phase, the decay of the miniemulsion polymerization rate is more like that of a homogeneous polymerization system. In macroemulsion polymerization, most of the CHP resides in the monomer droplets in the early stages of polymerization. CHP must continuously transfer from the droplets to the particles to react with TEPA, which would retard the TEPA consumption and prevent the TEPA exhausting seen in miniemulsion polymerization.

\section{Molecular Weight and Its Distribution}

The MWD development with conversion was monitored at two different initiator concentrations. The GPC spectra at low conversion are shown in Figures 8 and 9. For all GPC spectra shown, the contributions from the surfactant and initiator have been subtracted out. From Figures 8 and 9, it is clear that the spectra of polymer formed at the low conversion with the higher initiator level show three distinct peaks. The peak with highest retention volume is separate from the other two that partially overlap. The peak with highest retention volume has a molecular weight of approximately 1,000.

The multiple peaks could result from: (i) a time-cumulative effect, because the GPC sample represents an accumulation of product formed since the beginning of the reaction, or (ii) a space-cumulative effect, since, because of the multi-phase nature of macro- and miniemulsion polymerization, the GPC sample represents material formed in all phases. With a time-cumulative effect, the multi-peak effect should not be present at various 
monomer conversions. Since Figures 10 and 11 show that the multi-peak effect is persistent through changes in conversion, it is postulated that the multi-peak chromatograms represent a space-cumulative effect. As a matter of fact, macroemulsion polymerization is a heterogeneous system in which water-insoluble monomer and latex particles are dispersed in a continuous aqueous phase. It is assumed that the three peaks are derived from three different polymerization loci: the aqueous phase, the particle-water interface, and the particle interior.

The lowest molecular weight polymer is postulated to have been formed as oligomers in the aqueous phase. The highest molecular weight material is postulated to have been formed in the particle interior, and the intermediate molecular weight material formed as the interface. With these assumptions, from the peak area ratio and the monomer conversion, it is possible to determine the fraction of poly(butyl acrylate), which exists as oligomers formed in the aqueous phase. Figure 12 shows the oligomers concentration (expressed as total monomer conversion) as a function of total monomer conversion. A number of observations can be made: (i) At the beginning of polymerization, a great deal of oligomeric material is formed, and after that, the amount of oligomer formed increases steadily with conversion. (ii) With increase in initiator concentration, the amount of oligomer increases; at higher initiator concentration, the slope of the curve increases. (iii) The amount of oligomer formed at the beginning of miniemulsion polymerization is much less than that of macroemulsion polymerization; the slopes of the curves are lower in miniemulsion polymerization than in the macroemulsion polymerization; (iv) The intercept at zero conversion linearly increases with increase in initiator concentration (as shown in Figure 13). This can be well explained by the aqueous-phase polymerization kinetics, as shown in Appendix.

It is unexpected that much of the product at the beginning of polymerization results from the aqueous polymerization in that the ratio of $\mathrm{k}_{\mathrm{p}} / \mathrm{k}_{\mathrm{t}}$ is so high that even with a watersoluble inititator like potassium persulfate (KPS), initiation efficiency can be up to 99\% (Gilbert, 1995). This suggests the existence of other chain-stopping mechanisms (other than termination) such as chain transfer. This indication is in good agreement with the 
assumption that TEPA acts as a chain transfer agent in the present system. Because TEPA is hydrophilic, TEPA concentration would be high in the water phase, low in the particle interior, and intermediate at the interface. Because the TEPA concentration is high, and the BA concentration is very low in the water phase, some oligomers would form there, corresponding to the peak with highest elution time. Additionally, it is reasonable to assign the peak with middle elution time to polymer formed in the interface, and the peak with lowest elution time (highest molecular weight) to polymer formed in the interior of the particles. It is postulated that, at the beginning of polymerization, there are a large number of free radicals formed in the aqueous phase by reaction of TEPA with CHP (present at low levels in the aqueous phase) leading to high levels of oligomer formation. In addition, due to the large number of micelles present early in a macroemulsion, polymer will be formed in the precursor particles, then the radicals will be quickly desorbed due to chain transfer and desorption from the very small (high surface area) particles. The free radical concentration in the aqueous phase should be higher at the beginning of macroemulsion polymerization than at the beginning of miniemulsion polymerization due to the relative size of the initial particles. That is why the amount of oligomer formed early in miniemulsion polymerization is much less than in macroemulsion polymerization. In the middle and late stages of polymerization, the oligomers are much less likely to form because: (i) The free radical concentration is much reduced due to TEPA consumption. (ii) The TEPA concentration decays quickly so that oligomer formation by chain transfer is much less. (iii) Most free radicals can propagate into surface-active free radicals, and hence adsorb into particles. (iv) There are enough particles to capture the surface-active free radicals.

From Figures 10 and 11, it may be seen that the MWD data at higher conversion all show an unexpected behavior: an excess of low molecular weight species. A similar effect has been reported in methyl methacrylate and butyl methacrylate macroemulsion polymerization $[9,10]$. It has been suggested that this phenomenon may be due to a spatial inhomogeneity arising from surface anchoring. At relatively high conversion, monomer concentration in the particles is much lower, and so the radical cannot grow as quickly away from the surface. This causes a larger radical concentration in the outer 
portion of the particle compared to that deep within the particle. Clearly, this would lead to an increased rate of transfer to TEPA and an increase in the amount of low molecular weight species.

\section{$\underline{\text { Conclusions }}$}

From the present studies, it would seem that TEPA plays a double role: reducing agent in the redox initiator system and chain transfer agent. The unexpected data on monomer conversion and molecular weight is well explained if one postulates chain transfer via TEPA and considers the multi-phase nature of mini- and macroemulsion polymerization. Results for mini- and macroemulsion polymerization differ in ways which are explainable in terms of differences in particle size and number, particularly early in the reaction. It was suggested that the aqueous phase polymerization, interfacial polymerization and particle polymerization co-exist.

\section{Appendix}

At zero conversion, no particles exist. It is assumed that free radicals will form and terminate in the aqueous phase. A population balance of free radicals in the aqueous phase (neglecting, at early times, radical entry into micelles or droplets) gives

$$
\frac{d\left[M^{\bullet}\right]}{d t}=k_{d}[T E P A][C H P]-k_{t}\left[M^{\bullet}\right]^{2}
$$

Making the pseudo-steady state assumption, we have

$$
\left[M^{\bullet}\right]=\sqrt{\frac{k_{d}[T E P A][C H P]}{k_{t}}}
$$

and

$$
R_{p}=k_{p}[M]\left[M^{\bullet}\right]
$$

Substituting Eqn. (2) into Eqn. (3) gives

$$
R_{p}=k_{p}[M] \sqrt{\frac{k_{d}[T E P A][C H P]}{k_{t}}}
$$


Assuming a distribution coefficient $(\mathrm{k})$ relating aqueous and oil-phase concentrations for CHP gives:

$$
[\mathrm{CHP}]=\mathrm{k}[\mathrm{CHP}]_{\mathrm{o}}=\mathrm{k}^{\prime}[\mathrm{TEPA}]
$$

where the subscript "o" refers to the oil phase. Here, [TEPA] is linearly related to [CHP] (though $\mathrm{k}^{\prime}$ ), since, CHP and TEPA are added at a stoichiometric ratio of one, and, at early times, the effects of chain transfer have not substantially depleted TEPA. Combining Equations (4) and (5) gives

$$
\mathrm{R}_{\mathrm{p}}=\mathrm{K}[\mathrm{TEPA}]
$$

where $\mathrm{K}$ is a proportionality constant. From Equation (6), it is clear that the polymerization rate in the aqueous phase is linearly proportional to the initiator concentration, and in good agreement with the experimental data.

\begin{tabular}{|c|c|c|c|}
\hline Name & Acronym & Formula & Amount (g) \\
\hline Butyl acrylate & $\mathrm{BA}$ & $\mathrm{H}_{2} \mathrm{C}=\mathrm{CH}_{2} \mathrm{CO}_{2}\left(\mathrm{CH}_{2}\right)_{3} \mathrm{CH}_{3}$ & 75 \\
\hline Triton X-405 & & $4-\left(\mathrm{C}_{8} \mathrm{H}_{17}\right) \mathrm{C}_{6} \mathrm{H}_{4}-\left(\mathrm{OCH}_{2} \mathrm{CH}_{2}\right)_{\mathrm{n}} \mathrm{OH} \quad \mathrm{n}=40$ & 6.43 \\
\hline $\begin{array}{l}\text { Cumene } \\
\text { hydroperoxide } \\
(80 \%)\end{array}$ & CHP & $\mathrm{C}_{6} \mathrm{H}_{5} \mathrm{C}\left(\mathrm{CH}_{3}\right)_{2} \mathrm{OOH}$ & variable \\
\hline $\begin{array}{l}\text { Tetraethlene- } \\
\text { pentamine }\end{array}$ & TEPA & $\mathrm{HN}\left(\mathrm{CH}_{2} \mathrm{CH}_{2} \mathrm{NHCH}_{2} \mathrm{CH}_{2} \mathrm{NH}_{2}\right)_{2}$ & $\begin{array}{l}\text { Molar } \\
\text { equivalent } \\
\text { to CHP }\end{array}$ \\
\hline water & & $\mathrm{H}_{2} \mathrm{O}$ & 115 \\
\hline
\end{tabular}

Table 1. Basic Recipe 


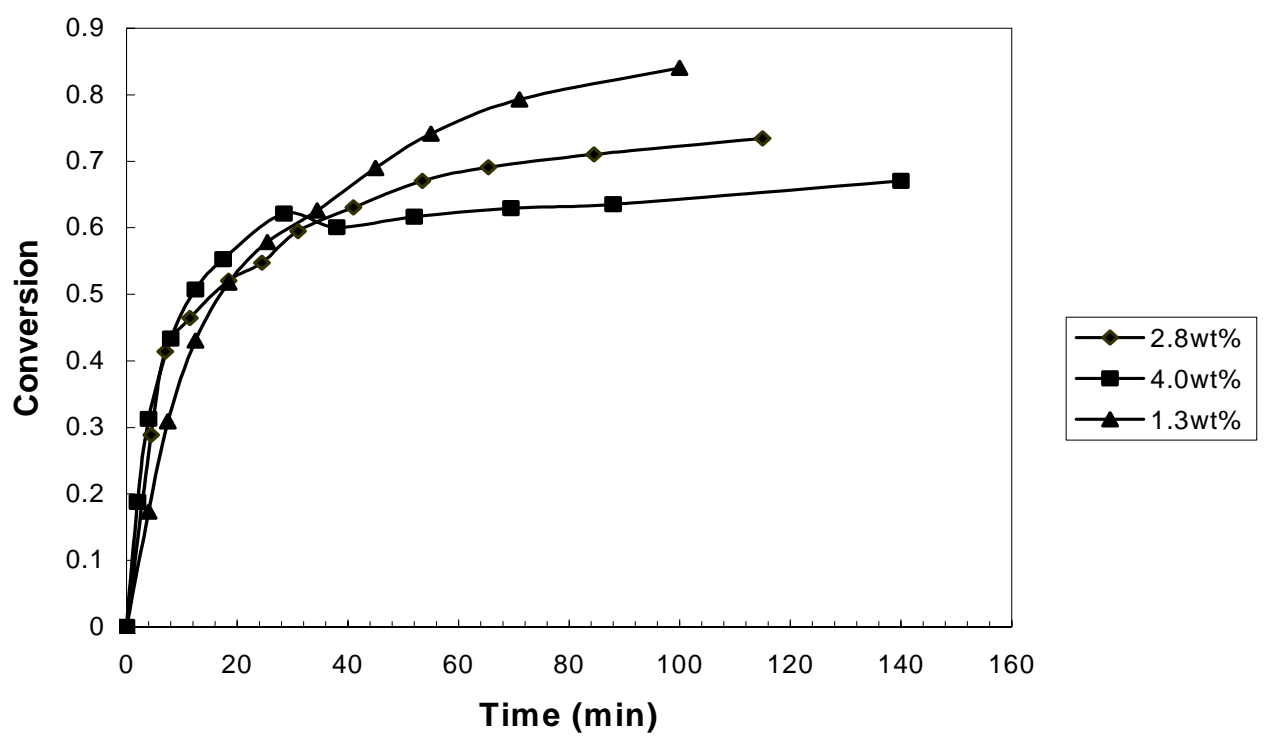

Figure 1. Conversion-Time Curves for Macroemulsion Polymerization with Varying Initiator Levels

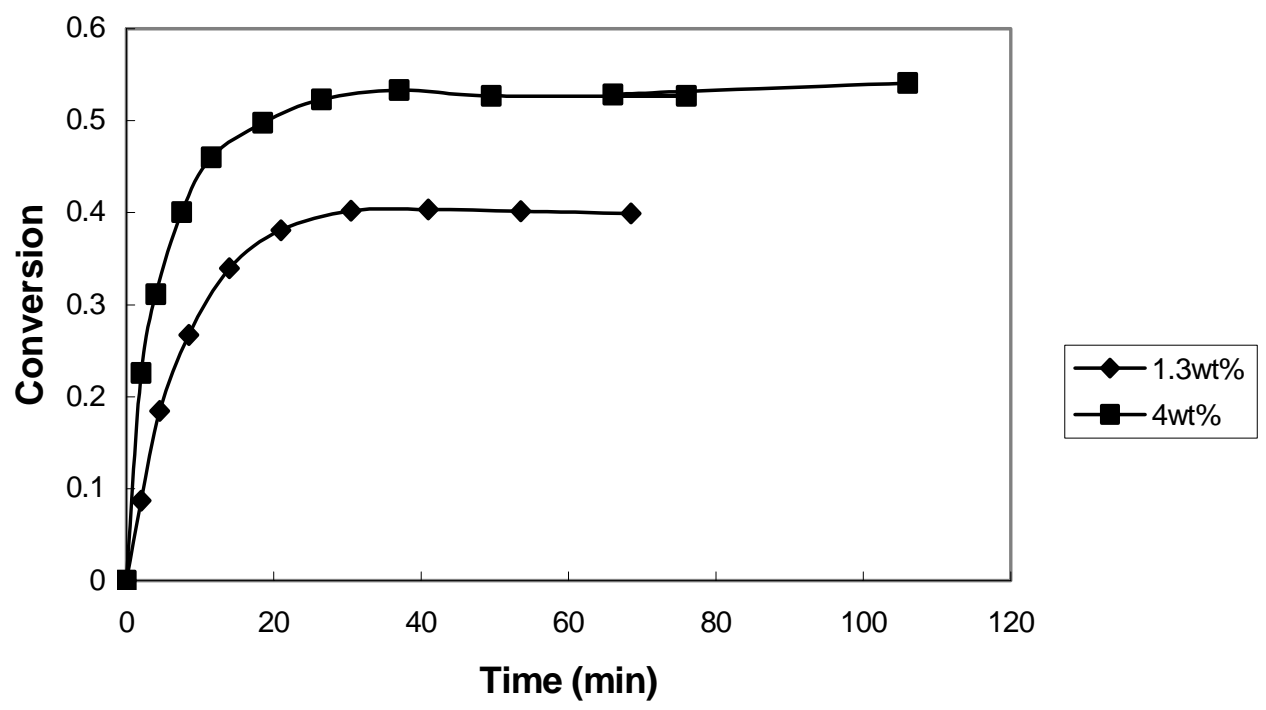

Figure 2. Conversion-Time Curves for Miniemulsion Polymerization with Varying Initiator Levels 


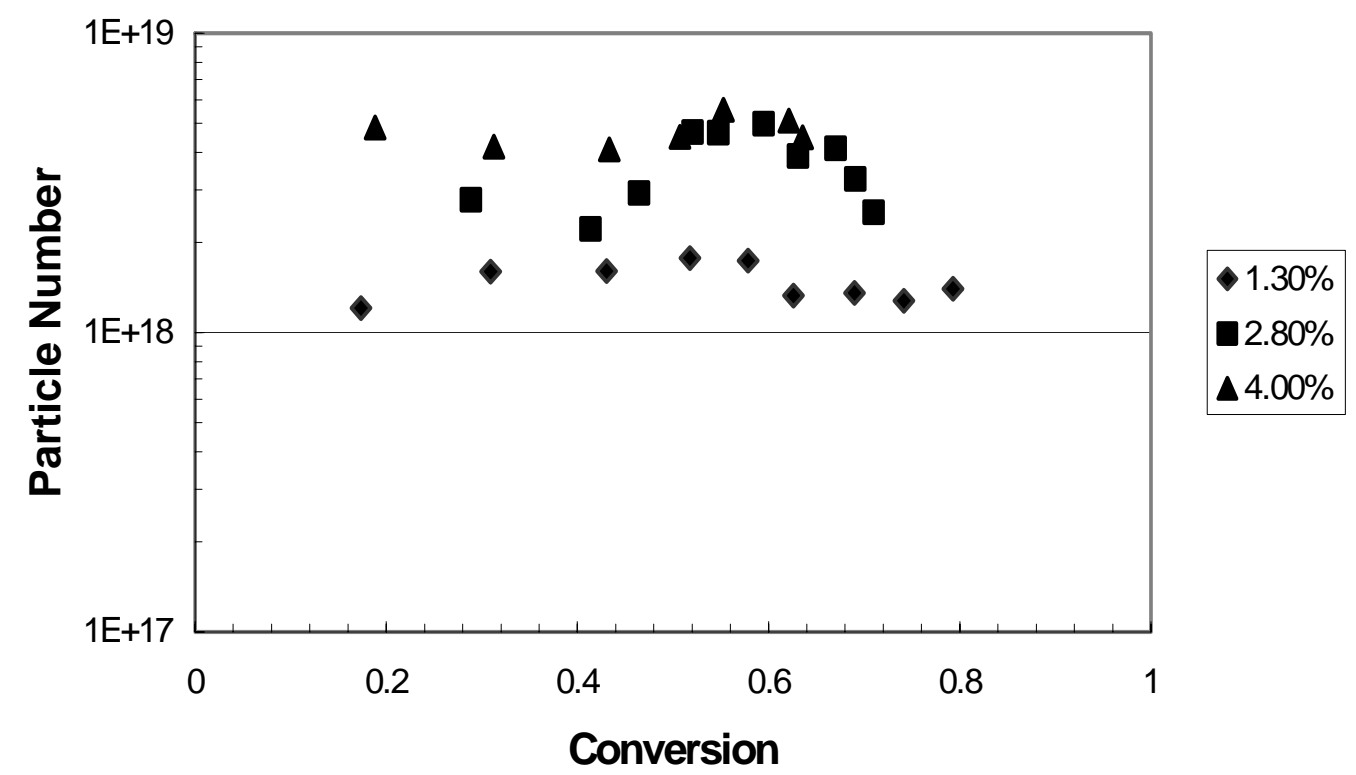

Figure 3. The Evolution of Particle Number with Conversion for Macroemulsion Polymerization with Varying Initiator Levels

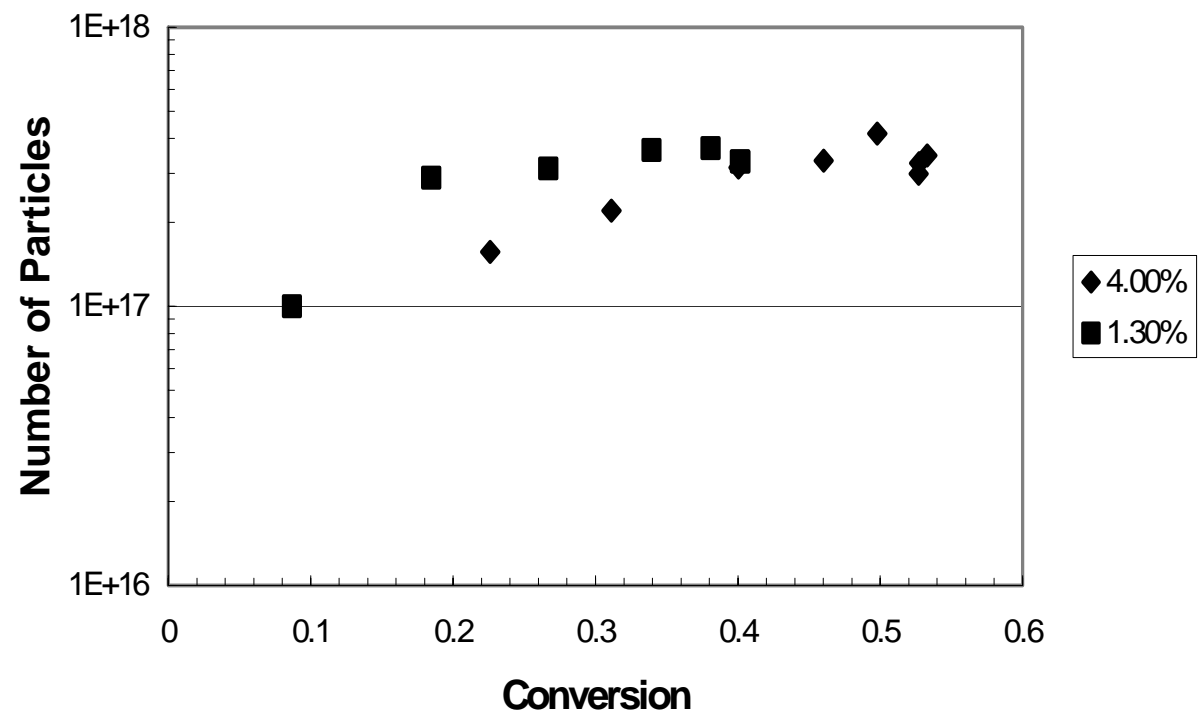

Figure 4. The Evolution of Particle Number with Conversion for Miniemulsion Polymerization with Varying Initiator Levels 


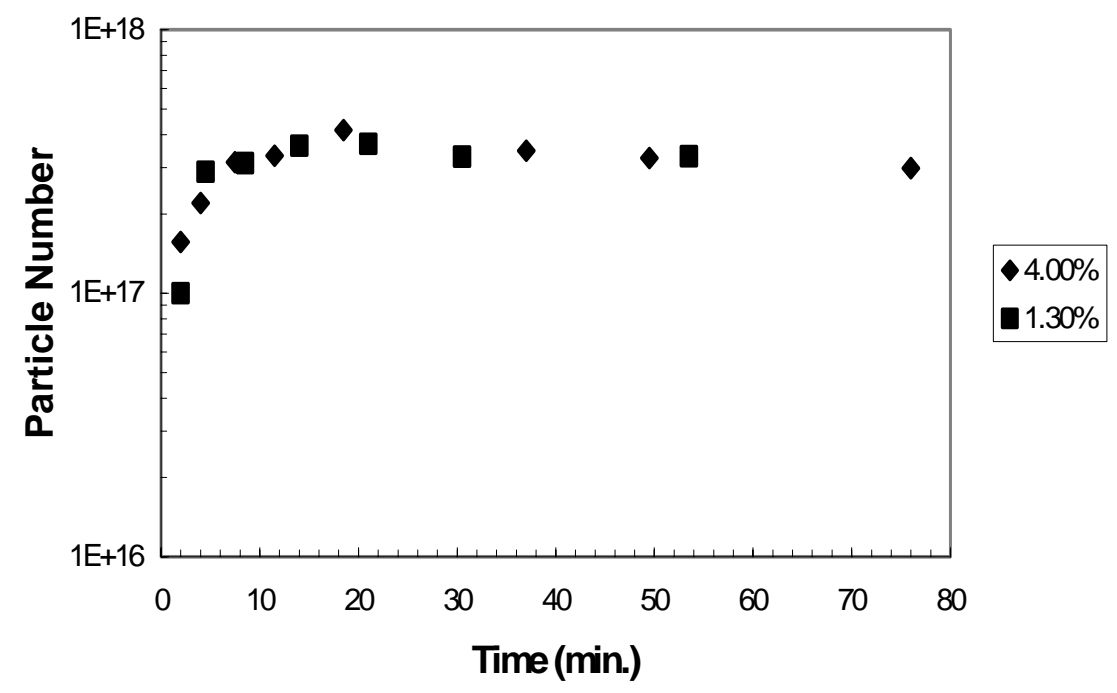

Figure 5. Particle Number Development with Time for Miniemulsion Polymerization with Varying Initiator Levels

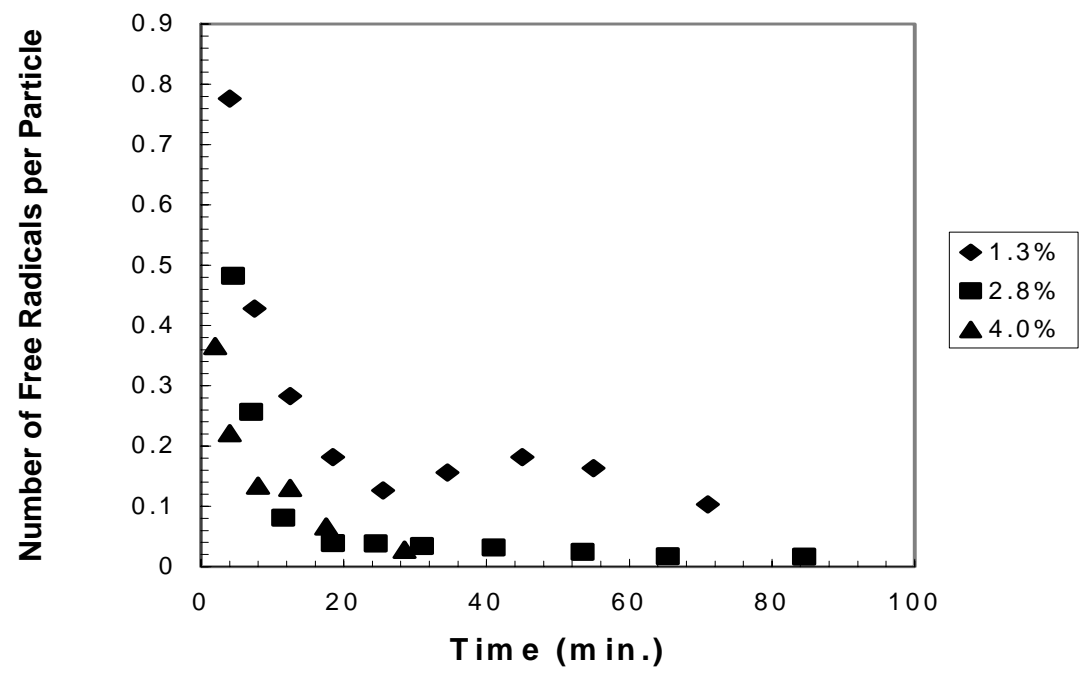

Figure 6. Number of Free Radicals Per Particle Development for Macroemulsion Polymerization with Varying Initiator Levels. 


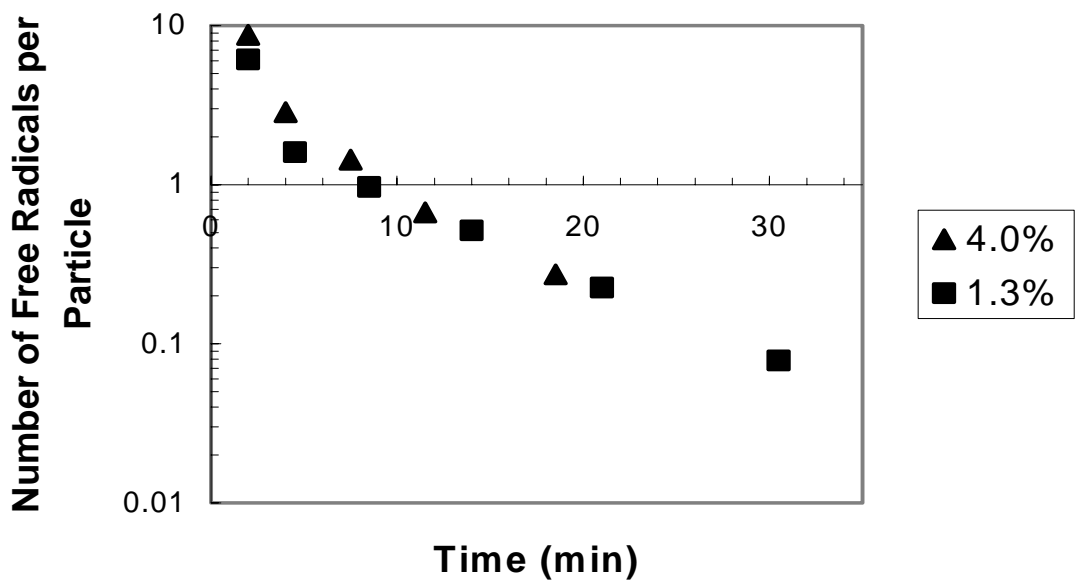

Figure 7. Number of Free Radicals per Particle for Miniemulsion Polymerization with Varying Initiator Levels.

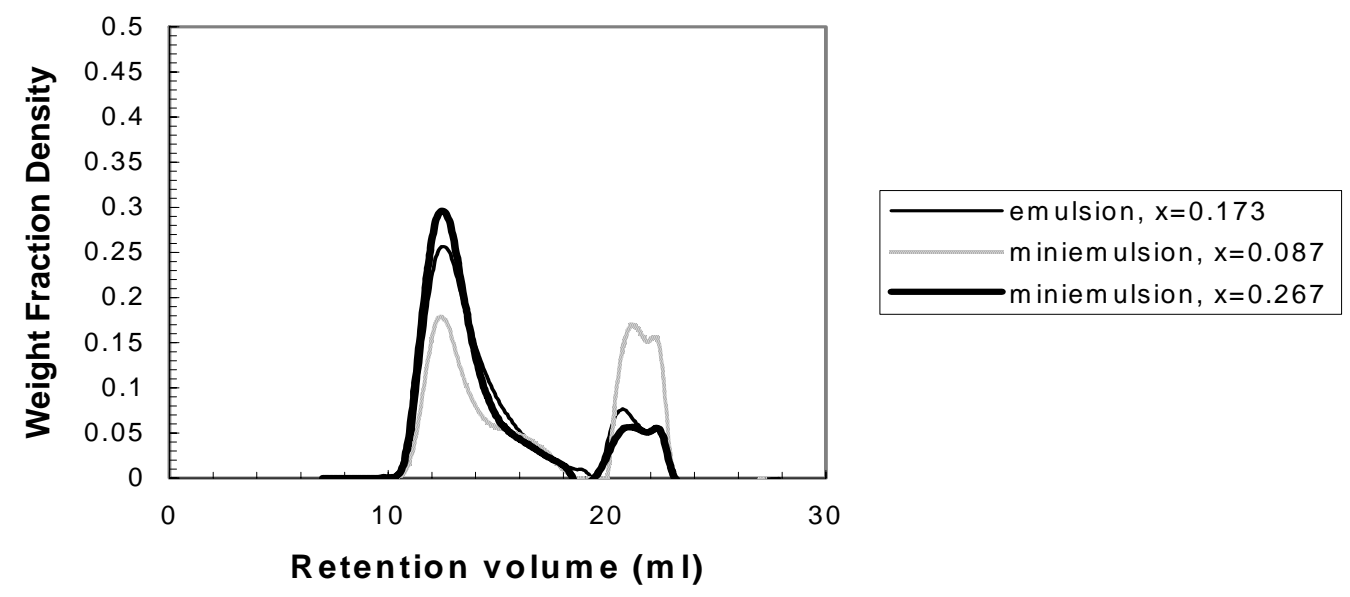

Figure 8 Molecular Weight Distribution of the Polymer from Macroemulsion and Miniemulsion Polymerization, Initiator $=1.33 \mathrm{wt} \%$ 


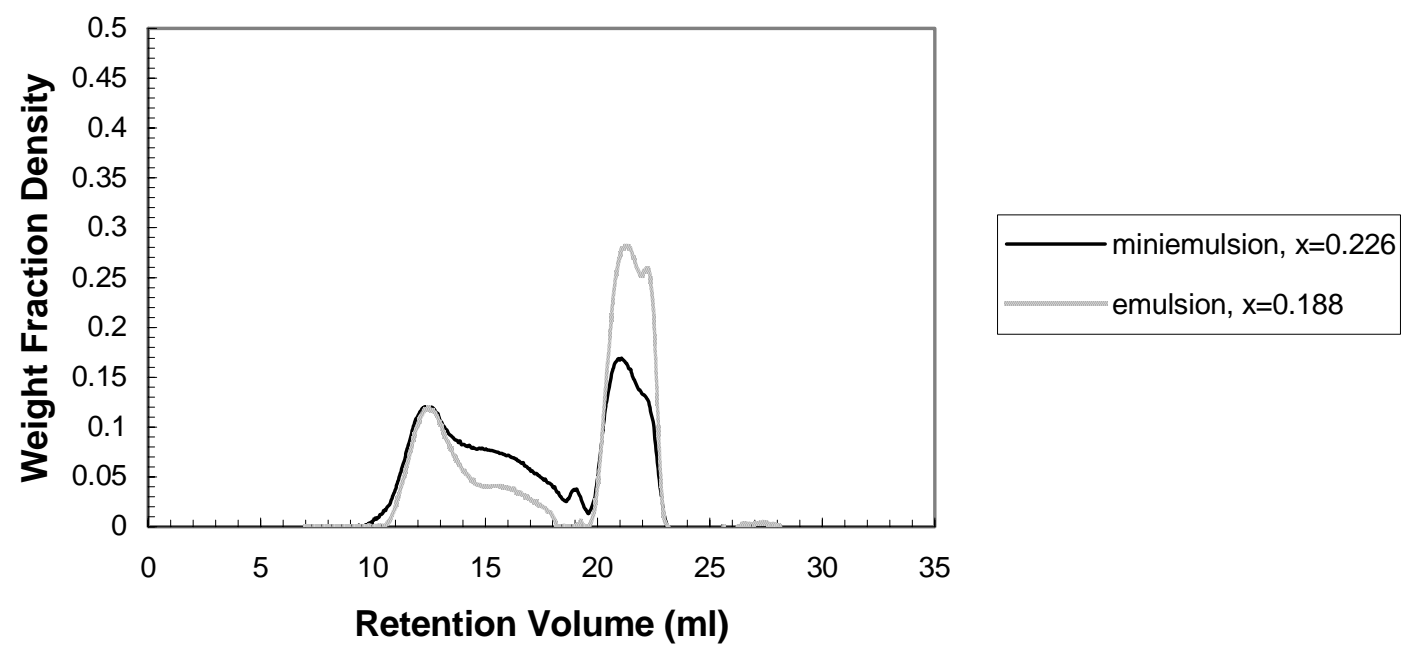

Figure 9 Molecular Weight Distribution of the Polymer from Macroemulsion and Miniemulsion Polymerization, Initiator $=4.0 \mathrm{wt} \%$

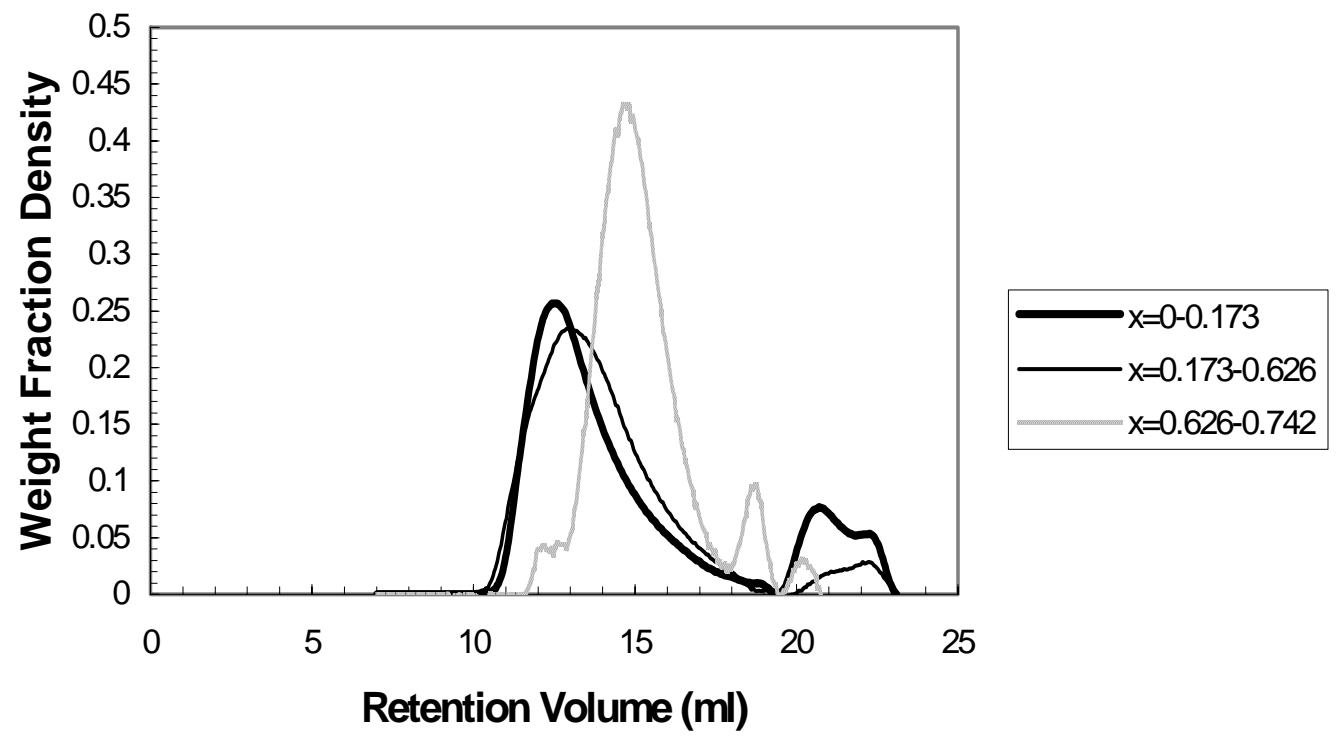

Figure 10. Molecular Weight Distribution Development with Conversion, Miniemulsion Polymerization, Initiator $=1.33 \mathrm{wt} \%$ 


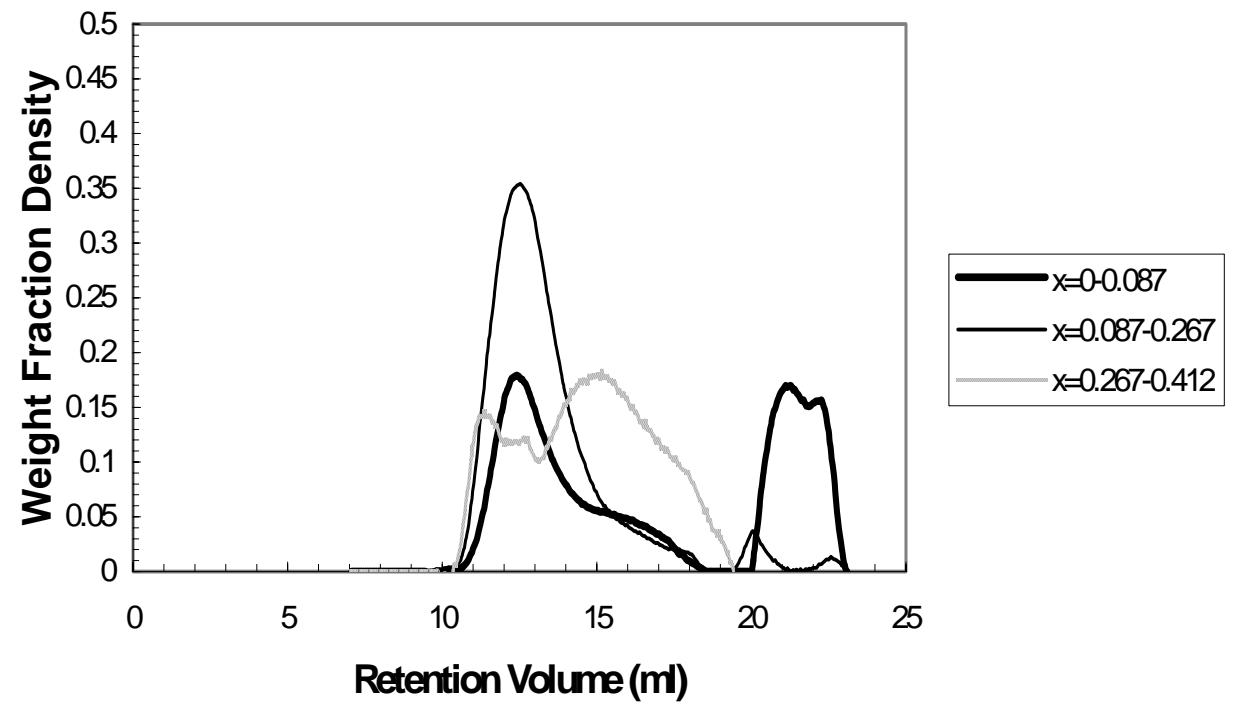

Figure 11. Molecular Weight Distribution Development with Conversion, Macroemulsion Polymerization, Initiator $=1.33 \mathrm{wt} \%$.

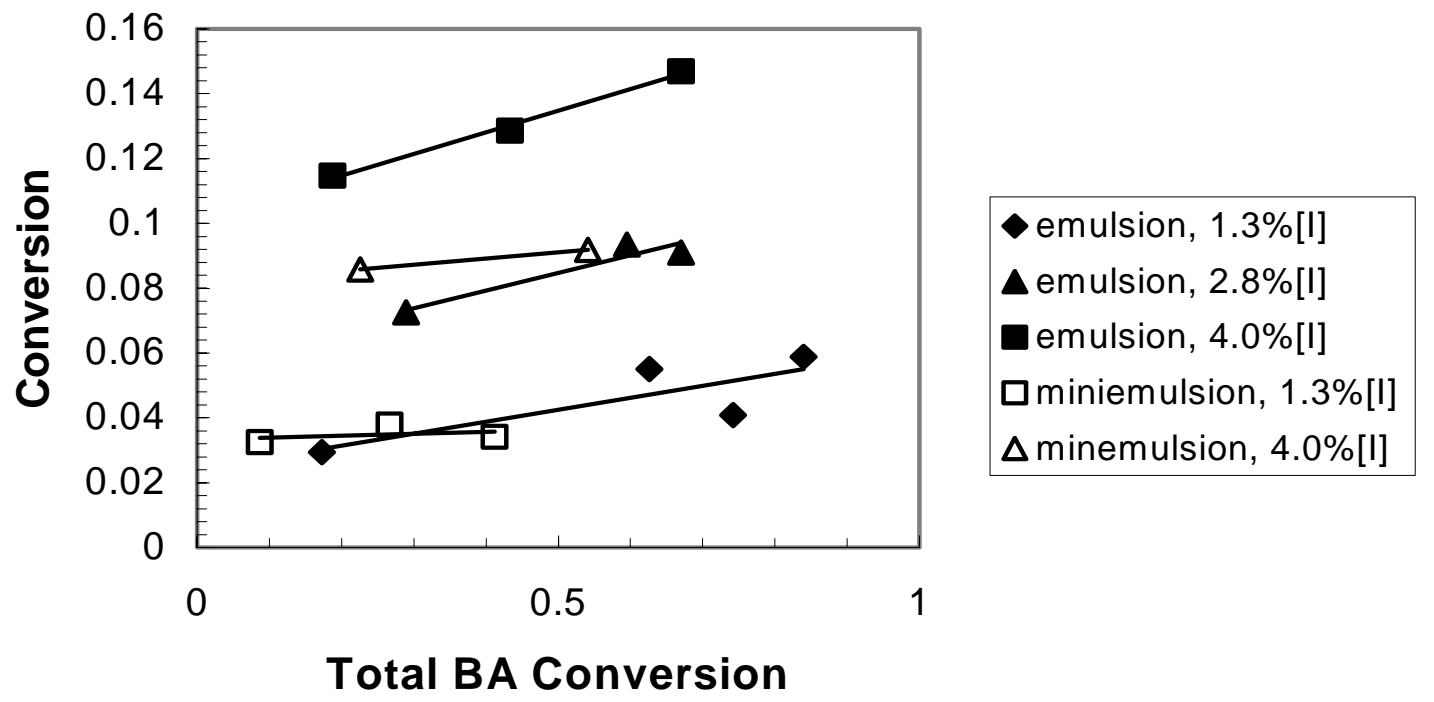

Figure 12. Conversion of BA Polymerized in the Aqueous Phase Versus Total Conversion 


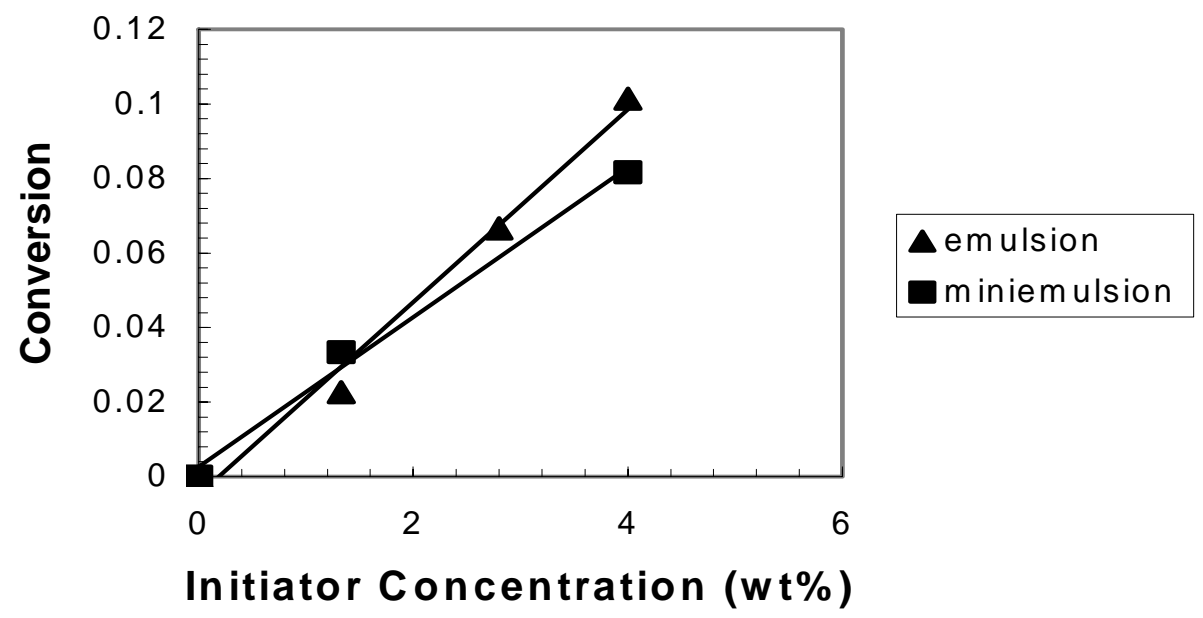

Figure 13. Correlation of Conversion of BA Polymerized in the Aqueous Phase with Initiator Concentration

\subsection{Emulsion Copolymerization of Butyl Acrylate with Cationic Monomer Using an Interfacial Redox Initiator System}

To copolymerize hydrophobic and cationic hydrophilic monomers by emulsion polymerization is a challenge, since the cationic monomer (in this study, [2(methacryloyoxy)ethyl]trimethyl ammonium chloride, MAETAC) will reside almost exclusively in the aqueous phase, while the hydrophobic monomer (in this study, butyl acrylate, BA) will reside almost exclusively in the organic (droplet or polymer particle) phase. Copolymerization of monomers with very different water-solubilities by emulsion technology has long been an active field of research, with the goal of synthesizing surface-functional polymer particles or increasing latex stability and mechanic properties of the polymer [1,3,11-17]. However, most of the studies so far are limited to a very low hydrophilic monomer level (less than $5 \mathrm{wt} \%$ ). In all cases, the incorporation of watersoluble monomer into the copolymer was very limited, regardless of the initial amount of water-soluble monomer loaded into the system. Kim [18] used a shot process to obtain a 
copolymer of styrene and sodium styrene sulphonate with a $6 \mathrm{wt} \%$ overall composition in a soap-free emulsion polymerization. Recently, Gilbert [1] proposed that by selecting an appropriate initiator system such as cumene hydroperoxide/tetraethylenepentamine (CHP/TEPA), it is possible to graft hydrophilic monomer onto a hydrophobic polymer in an emulsion process. For the current work, it was hoped that by using CHP/TEPA, the hydrophobic CHP would meet the hydrophilic TEPA at the particle/water interface where radicals would be produced at just the location where hydrophobic and hydrophilic monomer are both present. Siadat [2] reported that by the use of a two-component redox initiator, which was based on a water-soluble reducing agent and hydrophobic peroxide, a copolymer with moderate ionic monomer content could be prepared by an emulsion polymerization. However, Turner and co-workers [3] found that the redox initiator system did not increase the incorporation of sodium styrene sulfonate into polystyrene over that obtained with a typical water-soluble initiator. Siadat based his conclusion on the composition of the product at very low conversion (less than $2 \%$ ). Turner and coworkers drew their conclusion from the composition of the final product at high conversion (greater than 58\%). Clearly, more careful and extensive studies should be carried out to determine the role of two-component redox initiator system (one watersoluble, the other oil soluble), in an emulsion copolymerization of hydrophobic/hydrophilic monomer. In this part, polymerization kinetics of BA and MAETAC is investigated in order to add insight on the mechanism of this complex polymerization system.

\section{$\underline{\text { Experimental }}$}

Reagents

All agents were obtained from Aldrich Chemical. BA was purified by three washes with a $10 \% \mathrm{NaOH}$ solution followed by three washes with DI water. MAETAC was purified by three washes with anhydrous ether. All other reagents were used as received. Triton X-405 was used as surfactant. Hexadecane (HD) was used as cosurfactant in the miniemulsion polymerization 
The basic recipe is shown in Table 1 . A solution of the recipe amounts of MAETAC, DI water, Triton $\mathrm{X}-405$, and $\mathrm{NaCl}$ was mixed with a solution of the recipe amounts of $\mathrm{BA}$ and CHP (and HD in the cases of miniemulsion) under moderate shear. In the case of miniemulsions, the coarse emulsion was subjected to sonication for 15 minutes with a $300 \mathrm{~W}$ sonic dismembrator at 70\% power. Bulk mixing during sonication was provided by a magnetic stirrer. The macroemulsion (conventional emulsion without HD or sonication) or miniemulsion was added to the reactor and purged with nitrogen for a minimum of 45 minutes while the reactor temperature was raised from room temperature to $40{ }^{\circ} \mathrm{C}$. The polymerization was started by injecting the recipe amount of TEPA solution. Samples were drawn from the reactor at intervals and inhibited at intervals for subsequent analysis. Monomer conversion was determined gravimetrically. The $\mathrm{pH}$ remained at about 12 during the course of the polymerization.

NMR Analysis

The latex samples from the reactor were directly injected into a NMR tube filled with d4methyl alcohol after quenching with a hydroquinone solution. The samples were then analyzed in a $300 \mathrm{MHz}$ Varian NMR.

\section{Particle Size Analysis}

The latex was diluted with a Triton X-405 solution and held at $80{ }^{\circ} \mathrm{C}$ for more than 12 hours to drive off the unreacted BA. No evidence of polymerization or limited coagulation on heating was found. The apparent particle size was measured by dynamic light scattering with samples diluted with deionized water. A second measurement was made for each sample where the diluent was filtered $1 \mathrm{M} \mathrm{NaBr}$ solution. The later was used to collapse the layer of poly(MAETAC-BA) around the particles to get the true particle size. 
$\underline{\text { Results and Discussion }}$

The two monomers in the BA/MAETAC copolymerization are totally incompatible: BA is very hydrophobic and will reside primarily in the droplet/particle phase; MAETAC, being an ionic monomer, is very hydrophilic and will reside almost exclusively in the aqueous phase. The redox initiator system is composed of a hydrophobic oxidant (CHP), residing primarily in the droplet/particle phase, and a hydrophilic reductant (TEPA), residing primarily in the aqueous phase. These features make the emulsion polymerization rather complex because the multi-phase nature of the system. This part focuses on interfacial nature of the polymerization, and its effects on the kinetics of polymerization and properties of the latex.

\section{Evidence for Interfacial Initiation}

In a typical emulsion polymerization, initiator dissociates in the aqueous phase and then reacts with monomer dissolved in the water to form interfacially active free radical oligomers. These oligomers can be captured by particles or micelles and continue polymerization to high molecular weight in the organic phase. It has been stated that even with an oil-soluble initiator such as AIBN, most free radicals which generate polymer are actually formed in the aqueous phase from the decomposition of the small amount of AIBN dissolved in the water [19]; other researchers assert that the free radicals are formed in the particles [20]. For the system in question, it is important to determine if the polymerization is initiated by free radicals formed in the aqueous phase or those formed in the organic/aqueous interface, because free radicals formed in the aqueous phase would promote the formation of homopoly(MAETAC).

In order to investigate this question, a water-soluble initiator (VA-044) was used in the emulsion polymerization of BA/MAETAC (13.5 wt\% MAETAC based on MAETAC plus BA) with the nonionic surfactant Triton X-405. It was found that only homopoly(MAETAC) formed, and BA did not polymerize at all. Similar phenomena 
have been reported by Antonova [12] in studies of emulsion copolymerization of methacrylic acid and styrene with another nonionic surfactant OP-10. From these results, it would appear that no free radicals could enter a micelle to initiate organic-phase polymerization when a surfactant with a long hydrophilic tail was used. It is well known that only surface active free radicals in the water phase will be absorbed into the organic phase. ${ }^{15}$ Furthermore, it has been shown that the formation of the interfacially-active free radicals is a rate-determining step for the system with ionic surfactant such as SLS. However, for the system with a nonionic surfactant with a long hydrophilic tail such as Triton X-405 or OP-10, the particles or micelles would have a viscous corona, as shown in the Figure 1a, through which a surface active free radical must penetrate through before initiating organic phase polymerization. Kusters et al. [21] studied the effect of the corona consisting of PEO tail of degree of polymerization of 60 on the monomer radical exit. The exit coefficient was found to be an order magnitude lower in the case of nonionic surfactant as compared to an anionic emulsifier. However, Colombie et al. [22] found that no significant effect was noted on the radical entry rate coefficients within experimental error as compared surfactant sodium lauryl sulfate and Triton X-405. In both the above studies, the exit or entry species were small radicals. However, it has been reported that, in the presence of a highly water-soluble comonomer, the critical degree of polymerization for absorption into the organic phase is quite high [16]. With increase of degree of polymerization, the radical diffusion coefficient would be much lower. Based on the reptation model, the diffusion coefficient would decreases with the -2.0 power of degree of polymerization in semidilute and concentrated polymer solutions [23]. It is proposed that surface active free radicals formed in the aqueous phase (i) contain too low a BA content to become surface active, and (ii) have too high a degree of polymerization to successfully penetrate the viscous corona before termination in the aqueous phase.

By contrast, it was found that the BA in the BA/MAETAC copolymerization could be rapidly polymerized under the same conditions with the VA-044 replaced by CHP/TEPA. It would seem that initiation in the CHP/TEPA system occurs in the interface aqueous/organic interface so that a free radical need not penetrate through the surfactant layer before initiating BA, as shown in the Figure $1 \mathrm{~b}$. 
According to the reaction mechanism [18],

$$
\mathrm{ROOH}+\mathrm{R}^{\prime} \mathrm{NH}_{2} \rightarrow \mathrm{RO}^{\bullet}+\mathrm{R}^{\prime} \mathrm{NH}^{\bullet}+\mathrm{H}_{2} \mathrm{O}
$$

once CHP and TEPA meet at the aqueous/organic interface, two free radicals are formed, one hydrophilic and the other hydrophobic. It is reasonable to assume that two free radicals would separate from each other quickly because of their different nature so that the mutual termination often found with oil soluble initiators could be avoided.

\section{MAETAC Polymerization Kinetics}

Because of its highly heterogeneous nature, the product was difficult to separate and purify to get reliable data. Some authors have noted this problem and proposed procedures for doing so $[13,16]$, but for this study, instead of being separated, the latex sample from the reactor was directly injected into an NMR tube filled with d4-methyl alcohol after polymerization was quenched by an inhibitor solution. It is believed that ${ }^{1} \mathrm{H}$ NMR analysis should detect all MAETAC units, based on the following considerations: (i) a good dispersion was obtained; (ii) copolymer made by solution polymerization with $13.5 \mathrm{wt} \%$ MAETAC (based on total monomer) is soluble in methanol; (iii) with increased MAETAC composition in the copolymer, solubility in methanol is increased; (iv) copolymer macromolecules made by emulsion polymerization should be richer in MAETAC than those by solution polymerization because of the heterogeneous nature in the emulsion polymerization; (v) all copolymer should be near the surface of particles because of the high water solubility of MAETAC.

The resonance signals from protons of methyl groups connected to nitrogen (as shown in Figure 2) were used to determine MAETAC concentrations. MAETAC monomer exhibits a peak at $d=3.23$. Once the MAETAC is polymerized, two new resonance signals at $d=3.21 \mathrm{ppm}$ and $\mathrm{d}=3.36 \mathrm{ppm}$ were observed in the NMR. The signal from $\left(\mathrm{CH}_{3}\right)_{3} \mathrm{~N}^{+}$from homopoly(MAETAC) appears at $\mathrm{d}=3.21 \mathrm{ppm}$ and that of copolymer of BA/MAETAC appears at $d=3.36 \mathrm{ppm}$. The peak at $\mathrm{d}=3.21 \mathrm{ppm}$ is narrow, indicating a 
short relaxation time, typically from a low molecular weight component, whereas the peak at $d=3.36 \mathrm{ppm}$ is much broader, showing a longer relaxation time, typical of from nuclei on a polymer chain. Peak separation indicates that the nuclei are located in two different environments.

In view of its solubility in the aqueous phase, there are two potential polymerization loci for MAETAC: the aqueous phase or the interface between the aqueous and organic phases. As might be expected, the product from aqueous phase polymerization where the BA concentration is as low as $8.96 \mathrm{mM} / \mathrm{L}\left(50^{\circ} \mathrm{C}\right)$ [5] should be almost homopoly(MAETAC), and lower molecular weight as indicated by the fact that TEPA is a significant transfer agent [25]. The product of interfacial polymerization should have a higher level of BA incorporation and higher molecular weight. BA incorporation into poly(MAETAC), and higher molecular weight could significantly change the environment of MAETAC on the poly(MAETAC) because of changes in solubility and reduced salt dissociation. Based on these arguments, the peak at $\mathrm{d}=3.21 \mathrm{ppm}$ is assigned to oligomers of MAETAC formed in the aqueous phase and the peak at $\mathrm{d}=3.36 \mathrm{ppm}$ is assigned to polymer formed in the interface. These assignments will be further supported by the following kinetic study.

The MAETAC total conversion was calculated by:

$$
x_{2}=\frac{A_{2}+A_{3}}{A_{2}+A_{3}+A_{1}}
$$

and the MAETAC conversion in the polymerization of aqueous phase was calculated by:

$$
x_{2}^{\prime}=\frac{A_{2}}{A_{2}+A_{3}+A_{1}}
$$

where $A_{1}$ is the area of the peak at $d=3.23 \mathrm{ppm}, A_{2}$ is the area of the peak at $d=3.21$ $\mathrm{ppm}$, and $\mathrm{A}_{3}$ is the area of the peak at $\mathrm{d}=3.36 \mathrm{ppm}$. The conversion/time curves for macro- and miniemulsion polymerization are plotted in Figures 3 and 4. For both polymerizations, there is an initial burst of MAETAC polymerization. Somewhat more MAETAC is consumed at the very beginning of the macroemulsion polymerization than in the miniemulsion polymerization. The product believed to be formed in the aqueous 
phase [homo(MAETAC)] is mostly formed at the beginning of polymerization. For both the macro- and miniemulsion polymerization, the conversion of MAETAC in the aqueous phase remains constant at about $18 \%$ throughout the polymerization. The total MAETAC conversion increases steadily.

Before injecting TEPA to start polymerization, there is a small amount of CHP in the aqueous phase, since CHP has some water solubility. Once the TEPA is injected, a large number of free radicals form quickly in the aqueous phase. These free radicals will initiate MAETAC water-phase polymerization to produce almost homopoly(MAETAC). Because of the viscous surfactant layer and the increasingly swollen copoly(MAETAC) hydration layer, diffusion of additional CHP into the aqueous phase is retarded, and the CHP concentration in the aqueous phase fall rapidly. As a result, little homopoly(MAETAC) forms later in the polymerization. Early in a macroemulsion polymerization, a large number of micelles are present, each containing some BA. It is reasonable to assume that there should be some CHP dissolved in the micelles. The CHP contained in the micelles should be the first to react with TEPA, so that more MAETAC would be consumed at the very beginning of a macroemulsion polymerization than in a miniemulsion polymerization where no micelles exist. It is interesting to notice that although more MAETAC is consumed at the very beginning in the macroemulsion polymerization, the amount of homopoly(MAETAC) is almost equal for the macro- and miniemulsion polymerization. Once the CHP in a micelle reacts with TEPA, the local BA concentration is high, so that a copolymer will be formed, as was reported by Candau [26] in the micelle copolymerization of a water-soluble monomer and a water-insoluble monomer. The idea of micelle polymerization could also well explain the BA polymerization kinetics and will described in the following section.

From Figures 3 and 4, it can be seen that after the initiation burst, the MAETAC polymerization rate is almost constant in the macroemulsion polymerization while the MAETAC polymerization rate decreases with time in the miniemulsion polymerization. Theoretically, MAETAC polymerization rate in terms of conversion is a function of 
monomer concentration, free radical concentration at the polymerization locus, propagation rate constant, and the volume of the polymerization locus:

$$
\frac{d x_{2}}{d t}=k_{p} V\left[M_{2}\right]\left[M^{\bullet}\right]
$$

Where $\mathrm{x}_{2}$ is the MAETAC conversion, $\mathrm{k}_{\mathrm{p}}$ is the propagation rate constant, $\left[\mathrm{M}_{2}\right]$ is the MAETAC concentration at the interface, $\left[M^{*}\right]$ is thefree radical concentration at the interface, and $\mathrm{V}$ is the interface volume.

During Interval II of a macroemulsion polymerization, monomer concentration at the locus of polymerization is relatively constant. During miniemulsion polymerization, the monomer concentration at the locus of polymerization decreases continuously. Though it is difficult to determine the free radical concentration in the MAETAC polymerization zone, it is reasonable to assume that the evolution of the free radical concentration should be similar to that in a particle, which decreases with time. Since $k_{p}$ is not likely to change significantly, it is an increase of polymerization volume that should be responsible for the constant polymerization rate in the macroemulsion polymerization. This can be taken as evidence for interfacial polymerization since the particle surface area continuously increases in the macroemulsion polymerization whereas the volume of water phase cannot change at all. By contrast, for miniemulsion polymerization, the particle surface area changes much less so that the polymerization rate will continuously decrease, in good agreement with the model prediction.

From Figures 3 and 4, we can roughly estimate the error by NMR analysis. In the NMR analysis, the main errors should come from those MAETAC units which could not be detected by NMR, i.e. those buried in the particles. We have found that little of the MAETAC resides in organic phase, i.e. particles. Based on this, unreacted MAETAC peak area should be correct. The only error could be reduced detection of polymerized MAETAC. According to the results shown in Figures 3 and 4, the detected MAETAC conversion could be as high as $80 \%$. Based on the conversion trend, we should detect higher conversion at longer polymerization time. This means that the error should be much less than $20 \%$. 


\section{BA Polymerization Kinetics}

The BA polymerization kinetics were studied at various MAETAC levels. The results are presented in Figures 5 and 6. The effect of MAETAC on the BA polymerization kinetics is rather complicated. In comparison with homopolymerization of BA, adding $5 \mathrm{wt} \%$ MAETAC greatly increases the BA polymerization rate for both macroemulsion and miniemulsion polymerization. However, when the MAETAC level is increased further, the polymerization rate begins to decrease. At higher MAETAC levels, the effect of MAETAC is different for macroemulsion and miniemulsion polymerizations. For macroemulsion polymerization at high MAETAC level, there seems to be an induction period before polymerization begins. At higher MAETAC levels, the length of the induction period increases. For miniemulsion polymerization, there is no induction period. Instead, BA polymerization rate decreases to zero at less than full conversion. BA homopolymerization with CHP/TEPA levels off at approximately 30\% conversion. At higher MAETAC levels, the BA conversions levels off at substantially higher conversion.

\section{Particle Number}

Particle size analysis was accomplished using a Protein Solution LSR-TC dynamic light scattering instrument. The latex was diluted with a Triton X-405 solution and kept in an oven at $80^{\circ} \mathrm{C}$ for 12 hours to drive off the unreacted BA prior to analysis. It is postulated that copolymer formed at the interface will remain at the surface of the particle. To study the swelling of this interfacial layer, particle size measurements were carried out in pure water and $1 \mathrm{M} \mathrm{NaBr}$ aqueous solution. The interfacial layer should collapse in the presence a concentrated strong electrolyte aqueous solution. The results are listed in the Tables 2 and 3. It may be seen that at low MAETAC level or low monomer conversion, the apparent radius measured in pure water is the same as that in $1 \mathrm{M} \mathrm{NaBr}$ solution. At high MAETAC level and/or at high monomer conversion, the particle diameter is much larger when measured in pure water, indicating a thick layer of polyelectrolyte interfacial 
polymer, which can be collapsed by a electrolyte solution. It will be assumed that the radius measured in $\mathrm{NaBr}$ solution is the correct radius, and this was used to calculate particle number from the particle radius data. If the swollen radius used to calculate particle number, the particle number decreases with conversion, indicating particle aggregation. It is believed that for this system the particles should be sufficiently stable so that little coagulation is expected.

For comparison, $\mathrm{N}_{\mathrm{p}}$ for copolymerization as well as for $\mathrm{BA}$ homopolymerization is plotted versus BA conversion in Figures 7 and 8. It is evident that adding $5.0 \mathrm{wt} \%$ MAETAC led to more particles being formed both in the macroemulsion and miniemulsion polymerizations. This phenomenon has been ascribed to homogeneous nucleation. ${ }^{5}$ However, when the recipe MAETAC level is increased to $13.5 \mathrm{wt} \%$ the results are quite different from macroemulsion versus miniemulsion polymerization. For miniemulsion polymerization, the homogeneous nucleation effect disappears, as indicated by the fact that $\mathrm{N}_{\mathrm{p}}$ in the miniemulsion polymerization develops in exactly the same way as that of the miniemulsion homopolymerization, which leads to a polymerization rate of BA is not substantially affected by MAETAC level at higher MEATAC concentrations. It is evident that homogeneous nucleation is sensitive to the composition of homopoly(MAETAC). Contrary to El Aasser's observation [13] that homopoly(sodium styrene sulphonate) could initiate a homogeneous nucleation, for the present system, it seems that enough BA is incorporated into the poly(MAETAC) so that the copolymer undergoes homogeneous nucleation. For the macroemulsion copolymerization with 13.5 wt\% MAETAC, $\mathrm{N}_{\mathrm{p}}$ is even less than its homopolymerization counterpart. It is believed that $N_{p}$ is closely related to the induction period previously described. In fact, the induction period is not really a period of no polymerization, but rather a period of little BA polymerization. As shown in the previous section, a great deal of MAETAC was polymerized during the induction period and BA was also slowly polymerized up to approximately $10 \%$. This excludes the possibility that the induction period was brought about by some inhibitor impurity. Instead, because its miniemulsion polymerization counterpart showed no the "induction period", it is clear that at the very beginning of macroemulsion polymerization, micelles play a key role in the induction period. 
It is well accepted that hydrophobic molecules such as BA and CHP will be present in the micelles at the beginning of the polymerization. As described before, many of free radicals will be formed in the surface of a micelle and the two free radicals formed at the same time could separate each other quickly so that the so-called cage effect could be eliminated. In a micelle, the local BA concentration may be quite high. Once a micelle is initiated, a number of BA molecules may be added quickly. As a result, some short BA blocks would be incorporated into a poly(MAETAC) chain to form something like multiblock copoly(MAETAC-BA), as shown in Figure 9. Surfactant should stabilize the BA blocks so that the block copolymer would remain in the aqueous phase. This idea is further supported by the observances that most of the product at the very beginning of polymerization is water soluble, and that the strong resonance signals of ${ }^{1} \mathrm{H}$ - NMR from polymerized BA can be detected in $\mathrm{D}_{2} \mathrm{O}$. The BA blocks in the copolymer, which is surrounded by surfactant, would swell with BA monomer, which would polymerize there until particles form. In the meanwhile, micellar nucleation would be diminished or even eliminated. As a result, micellar nucleation would be retarded, and $\mathrm{N}_{\mathrm{p}}$ decreased.

\section{Average Number of Free Radical Per Particle}

By combining conversion versus particle versus time data, the average number of free radicals in a particle was calculated as [5]:

$$
\bar{n}=\frac{R_{p} N_{a v} n_{M, 0}}{k_{p} C_{p} N_{p}}
$$

Where $\mathrm{k}_{\mathrm{p}}$ is the propagation rate constant of $\mathrm{BA}$, calculated by $\mathrm{k}_{\mathrm{p}}=1.874 \mathrm{e} 11^{*} \exp (-$ 7026.7/T), L/mol-s [6]; $\mathrm{C}_{\mathrm{p}}$ is the $\mathrm{BA}$ concentration in a particle, $4.57 \mathrm{~mol} / \mathrm{L}$ in the Intervals I and II in the macroemulsion polymerization, otherwise a function of BA conversion; $\mathrm{N}_{\mathrm{p}}$ is the particle number in the system per unit volume of aqueous phase; $\mathrm{N}_{\mathrm{av}}$ is Avogadro's Number; $\mathrm{N}_{\mathrm{M}, 0}$ is the initial number of moles of monomer per unit volume of water 
Figures 10 and 11 show the evolution of the average number of free radicals per particle $(\bar{n})$. From these figures, it is clear that in the copolymerization system, $\bar{n}$ is greater than in homopolymerization, and with increasing MAETAC level, $\bar{n}$ increases. In the literature, the effect has been ascribed to a gel effect caused by ionic crosslinking [3]. Once MAETAC ionic monomer is incorporated into the BA polymer chains, some ionic clusters are formed and function as crosslinks. These crosslinking points would hinder termination of free radicals so as to increase $\bar{n}$. However, because of the extensive exit of free radicals (due to the chain transfer effect of TEPA) [25], the controlling mechanism in the determination of $\bar{n}$ is the exit rather than the termination of free radicals. It is proposed that the increase in $\bar{n}$ should be ascribed to the hindrance of radical exit due to the hydration layer.

The effect of the hindrance can also been seen in the decay of $\bar{n}$ with conversion. From Figures 10 and 11, the decay of $\bar{n}$ is slower in the copolymerization systems than that of the homopolymerization systems. Furthermore, the rate of decay decreases with increase in MAETAC level. Because the decay rate is determined by the consumption of TEPA [25], it may be inferred that the TEPA consumption rate is slower during the copolymerization. It may be concluded that the hydration layer hinders TEPA transfer from the aqueous phase to the particle surface where it is consumed. It is this hindrance that allows the BA polymerization to proceed for a longer time (before being totally consumed) in the copolymerization than the equivalent homopolymerization.

\section{Copolymer Composition and Properties of the Hydration Layer}

Because of the heterogeneous nature of the system, the composition of copolymer formed in the interface is very difficult to determine. Instead, the hydration properties of the interfacial layer will be used to infer copolymer composition at the interface. As shown in the Tables 2 and 3, the apparent radii of the copolymer latex measured in DI water and 1 $\mathrm{M} \mathrm{NaBr}$ solution are substantially different. This difference has been to use to derive a new parameter defined as the degree of swelling Q. The hydration properties of the 
interfacial layer are determined by balancing the chemical potential of water in and out of swollen layer [27], i.e.

$$
\Delta \mu=\Delta \mu^{e l a}+\Delta \mu^{p o l}+\Delta \mu^{i o n}=0
$$

where $\Delta \mu^{e l a}, \Delta \mu^{\text {pol }}$, and $\Delta \mu^{\text {ion }}$ are elastic, polymer/water-mixing, and ion/water-mixing contribution to the chemical potential, respectively. When the sum of $\Delta \mu^{\text {pol }}$ and $\Delta \mu^{\text {ion }}$ are offset by $\Delta \mu^{e l a}$, an equilibrium is establishjed. $\Delta \mu^{e l a}$ is detemined primarily by the effective crosslink density. In the present case, crosslinking points come from anchoring of hydrophobic segments in copolymer chains or hydrophobic aggregation between copolymer chains. With increased salt concentration, the contribution from $\Delta \mu^{\text {ion }}$ decrease sharply. In a high salt concentration solution, the driving force for swelling is mainly from $\Delta \mu^{\text {pol }}$. In a salt-free medium, the driving force for swelling is mainly from $\Delta \mu^{i o n}$. In a salt-free medium, far more water will be adsobed into the hydration layer than in a high salt medium. Copolymer composition will influence the hydration properties in the following ways:

- With an increase in BA content, the copolymer has more hydrophobic units as a crosslinking point which affects $\Delta \mu^{e l a}$ so that degree of swelling will decrease

- With an increase of BA content, the copolymer/water interation parameter will change so that $\Delta \mu^{\text {pol }}$ and consequesntly the degree of swelling will decrease.

Clearly, the hydration properties in a salt-free enviroment is highly sensitive to BA composition.

The difference of apparent radius between a salt-free medium and the true radius of a dry particle should represent the hydration efficiency of the copolymerized MAETAC. For convenience, a new parameter degree of swelling is defined as

$$
Q=\frac{0.75 \pi\left(r_{2}^{3}-r_{1}^{3}\right) \times N_{p} \times d_{0}}{W_{2,0}\left(x_{2}-x_{2}^{\prime}\right)}
$$

Where $r_{2}$ is the apparent radius from a salt-free medium; $r_{1}$ is the apparent radius in a 1 $\mathrm{M} \mathrm{NaBr}$ solution (as an approximation, the radius of a dry particle is replaced by the apparent radius of a particle in $1 \mathrm{M} \mathrm{NaBr}$ solution because they are very close.); $\mathrm{N}_{\mathrm{p}}$ is the 
particle number in the systems; $d_{0}$ is the density of $\mathrm{H}_{2} \mathrm{O} ; \mathrm{W}_{2,0}$ is the recipe weight of MAETAC; $\mathrm{x}_{2}$ is the total conversion of the MAETAC; $\mathrm{x}_{2}$ ' is the conversion of MAETAC from reaction in the water, which is not considered to contribute to the hydration layer.

It is clear from the equation that $\mathrm{Q}$ represents the hydration capacity of polymerized MAETAC fixed on the surface of the particle in a pure water environment. It is also clear that the larger $\mathrm{Q}$, the lower the BA composition in the interfacial layer. The degree of swelling as a function of BA conversion is plotted in Figure 12 for macro- and miniemulsion polymerization. It is seen that during the Intervals I and II, the Q value is close to zero. However, once the polymerization proceeds into Interval III, the Q value increases rapidly. The large change in Q value should be ascribed to crosslinking density development, i.e. BA content development. In contrast, homopoly(MAETAC) prepared by aqueous solution polymerization in the presence of a small amount of BA was mixed with homopoly(BA) latex. It is found that the particle size distribution was nearly identical for homopoly(BA) latex before and after mixing with homopoly(MAETAC). This experiment indicates that homopoly(MAETAC) formed in the water phase cannot effectively be adsorbed onto the surface of the particle to form a hydration layer under the condition of the measurement. The hydration layer only comes from the interfacial polymerization.

From Figure 12, it may be inferred that the copolymer formed during Intervals I and II has a higher BA content, while during Interval III the newly formed copolymer is lower in BA content. This inference is in good agreement with BA monomer concentration development in macroemulsion polymerization. In Intervals I and II, the fact that the particles are saturated with BA (because of the presence of droplets in macroemulsion polymerization) keeps a relatively high concentration on the surface of the particles whereas in Interval III the particles are no longer saturated with BA, and the BA concentration in the interfacial layer falls. It is interesting to note that the critical point where the $\mathrm{Q}$ value begins to dramatically increase is different for miniemulsion and macroemulsion polymerization. There are two possible reasons for this: (i) Equilibrium measurements [6] indicate that Interval III should begin at a BA conversion of $40 \%$, 
while kinetic data on macroemulsion polymerization indicate that Interval III should begin at 56\% [7]. In contrast, there is no Interval II in miniemulsion polymerization, since the monomer concentration in the particles decreases monotonically from the beginning of polymerization. (ii) Because the particles formed in macroemulsion polymerization are smaller than those in miniemulsion polymerization, there is much more interfacial area to provide more crosslinking points. If the copolymer had the same composition, the $\mathrm{Q}$ value of the macroemulsion polymerization latex would be lower than that of the miniemulsion polymerization.

When compared with those of macroemulsion polymerization, the $\mathrm{Q}$ values of miniemulsion polymerization are higher, indicating a lower BA content in the copolymer formed during Interval III. This can be explained by the difference in particle size. In miniemulsion polymerization, the particles are larger than those in macroemulsion polymerization, so that BA monomer in the bulk of particle is less likely to be at the surface; this lead to a lower BA content in the copolymer formed.

When salt is added to the emulsion prior to polymerization, the $\mathrm{Q}$ value increases and the critical point appears at lower BA conversion, indicating that a lower BA copolymer formed has been formed at the interface (Figure 12, miniemulsion, 13.5\%, with salt). It has been reported that when the ionic strength of the aqueous phase is increased, the concentration of hydrophobic monomer at the surface of a micelle decreases [28]. A similar effect should occur for particles in this study, which explains why polymer with a lower BA content is formed in the presence of salt.

\section{$\underline{\text { Conclusions }}$}

The copolymerization of BA and MAETAC can be accomplished using the interfacial redox initiator system CHP/TEPA. The following conclusions can be drawn from this work:

1. The nucleation process is very strongly dependant on MAETAC concentration and polymerization method. For miniemulsion polymerization, homogeneous 
nucleation occurs at low MAETAC concentration but disappears at higher MAETAC concentration. For macroemulsion polymerization, the nucleation is complicated because of the depletion of micelles to form temporarily watersoluble polymer.

2. Interfacial polymerization kinetics is dependent on polymerization method and the level of salt addition.

3. For both miniemulsion and macroemulsions, approximately $18 \%$ of the total MAETAC is polymerized in the water phase early in the reaction.

4. The copolymer produced from these polymerizations is of a very heterogeneous in composition

5. The copolymer composition is significantly influenced by particle size, conversion, and salt addition.

Table 1. Basic Recipe

\begin{tabular}{lll}
\hline Name & Acronym & Amount (g) \\
\hline Butyl acrylate & BA & 75 \\
$\begin{array}{l}\text { [2-(Methacryloxy)ethyl] } \\
\text { trimethyl ammonium chloride } \\
\text { Triton x-405 }\end{array}$ & MAETAC & Variable \\
Cumene hydroperoxide 80\%) & CHP & 6.43 \\
Tetraethylenepentamine & TEPA & 1.0 \\
De-ionic water & & 1.0 \\
Sodium Chloride (when used) & $\mathrm{NaCl}$ & 115 \\
\hline
\end{tabular}


Table 2. Particle Number Development in Miniemulsion Copolymerization

\begin{tabular}{|l|l|l|l|l|l|l|l|}
\hline \multicolumn{3}{|l|}{ 5.0wt\% MAETAC } & \multicolumn{3}{l|}{ 13.5wt\% MAETAC } \\
\hline $\mathrm{x}_{1}$ & $\mathrm{r}_{1}(\mathrm{~nm})$ & $\begin{array}{l}\mathrm{r}_{2}(\mathrm{~nm} \\
)\end{array}$ & $\mathrm{N}_{\mathrm{p}}$ & $\mathrm{x}_{1}$ & $\begin{array}{l}\mathrm{r}_{1}(\mathrm{~nm} \\
)\end{array}$ & $\mathrm{r}_{2}(\mathrm{~nm})$ & $\mathrm{N}_{\mathrm{p}}$ \\
\hline 0.120 & 57.11 & 55.43 & $1.08 \mathrm{E} 16$ & 0.133 & 60.62 & 61.99 & $1.32 \mathrm{E} 16$ \\
\hline 0.265 & 53.12 & 54.72 & $2.96 \mathrm{E} 16$ & 0.214 & 66.4 & 68.31 & $1.51 \mathrm{E} 16$ \\
\hline 0.511 & 63.63 & 61.28 & $3.32 \mathrm{E} 16$ & 0.329 & 71.77 & 80.26 & $1.75 \mathrm{E} 16$ \\
\hline 0.684 & 67.09 & 68.23 & $3.81 \mathrm{E} 16$ & 0.464 & 85.58 & 136.9 & $1.42 \mathrm{E} 16$ \\
\hline 0.802 & 70.42 & 71.67 & $3.84 \mathrm{E} 16$ & 0.584 & 88.00 & 202.32 & $1.63 \mathrm{E} 16$ \\
\hline 0.856 & 74.30 & 73.23 & $3.49 \mathrm{E} 16$ & 0.654 & 93.22 & 225.20 & $1.54 \mathrm{E} 16$ \\
\hline 0.878 & 73.07 & 77.77 & $3.76 \mathrm{E} 16$ & 0.715 & 91.54 & 257.97 & $1.77 \mathrm{E} 16$ \\
\hline 0.883 & 72.88 & 74.38 & $3.82 \mathrm{E} 16$ & 0.778 & 92.95 & 298.31 & $1.83 \mathrm{E} 16$ \\
\hline & & & & 0.815 & 95 & 283.89 & $1.80 \mathrm{E} 16$ \\
\hline
\end{tabular}

Note: $x_{1}$ is $B A$ conversion; $r_{1}$ is the apparent particle radius in salt solution, $r_{2}$ is the apparent particle radius in DI water

Table 3. Particle Number Development in Macroemulsion Copolymerization

\begin{tabular}{|l|l|l|l|l|l|l|l|}
\hline \multicolumn{4}{|l|}{$5.0 \mathrm{wt} \%$ MAETAC } & \multicolumn{4}{l|}{ 13.5wt\% MAETAC } \\
\hline $\mathrm{x}_{1}$ & $\mathrm{r}_{1}(\mathrm{~nm})$ & $\mathrm{r}_{2}(\mathrm{~nm})$ & $\mathrm{N}_{\mathrm{p}}$ & $\mathrm{x}_{1}$ & $\mathrm{r}_{1}(\mathrm{~nm})$ & $\mathrm{r}_{2}(\mathrm{~nm})$ & $\mathrm{N}_{\mathrm{p}}$ \\
\hline 0.225 & 27.34 & 32.76 & $1.07 \mathrm{E} 17$ & 0.079 & 43.46 & 44.54 & $3.35 \mathrm{E} 16$ \\
\hline 0.375 & 34.38 & 35 & $1.40 \mathrm{E} 17$ & 0.150 & 45.16 & 45.26 & $4.35 \mathrm{E} 16$ \\
\hline 0.620 & 41.00 & 43.87 & $1.23 \mathrm{E} 17$ & 0.335 & 53.1 & 53.26 & $4.83 \mathrm{E} 16$ \\
\hline 0.796 & 44.09 & 50.42 & $1.04 \mathrm{E} 14$ & 0.532 & 63.1 & 87.59 & $4.23 \mathrm{E} 16$ \\
\hline 0.881 & 51.82 & 60.15 & $6.78 \mathrm{E} 16$ & 0.689 & 70 & 137.3 & $3.94 \mathrm{E} 16$ \\
\hline 0.929 & 54.42 & 61.8 & $6.59 \mathrm{E} 16$ & 0.763 & 78.85 & 172.91 & $3.04 \mathrm{E} 16$ \\
\hline 0.958 & 52.71 & 81.77 & $2.94 \mathrm{E} 16$ & 0.831 & 97.93 & 210.04 & $1.73 \mathrm{E} 16$ \\
\hline & & & & 0.886 & 101.5 & 246.00 & $1.65 \mathrm{E} 16$ \\
\hline
\end{tabular}

Note: $x_{1}$ is $B A$ conversion; $r_{1}$ is the apparent particle radius in salt solution, $r_{2}$ is the apparent particle radius in DI water 


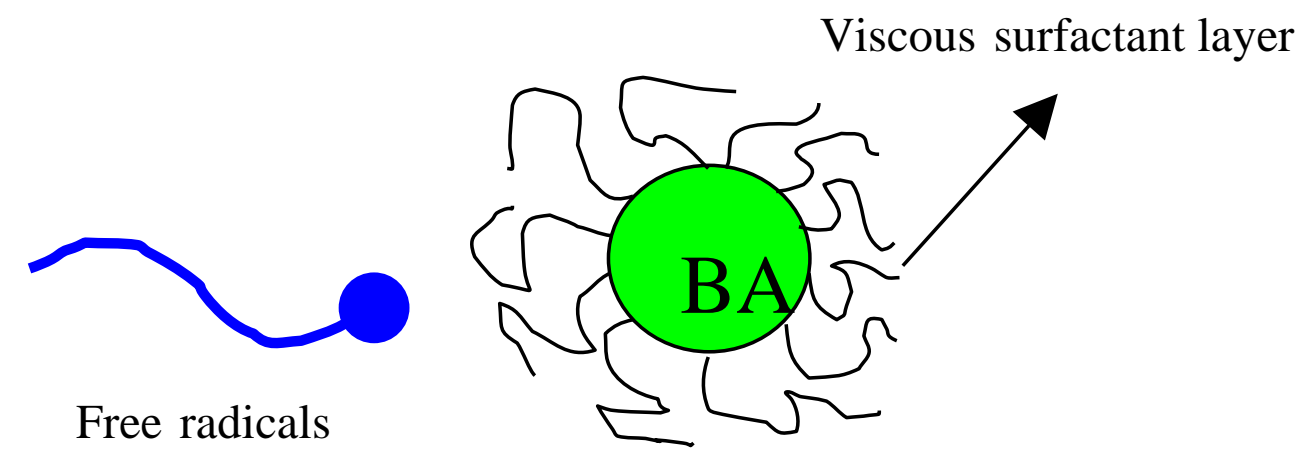

Micelle

Fig. 1a

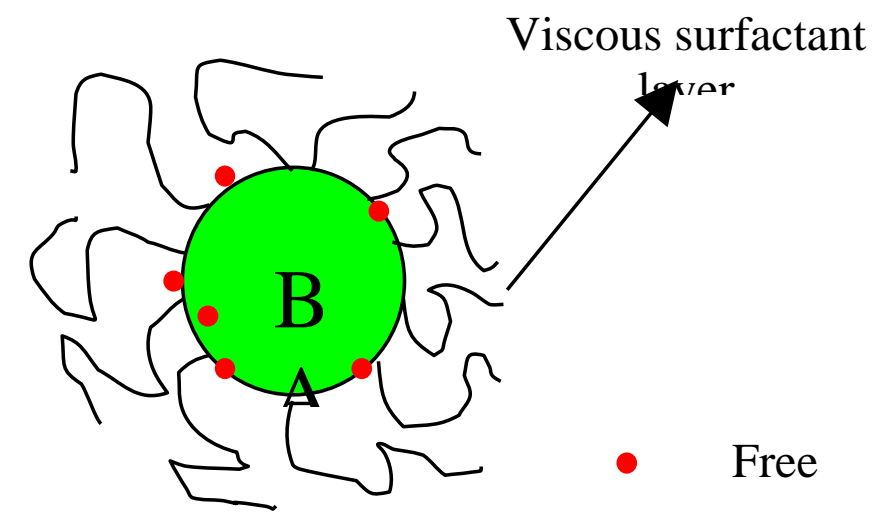

Mice

Fig. 1b

Figure1. Initiation with a Polymeric Surfactant 

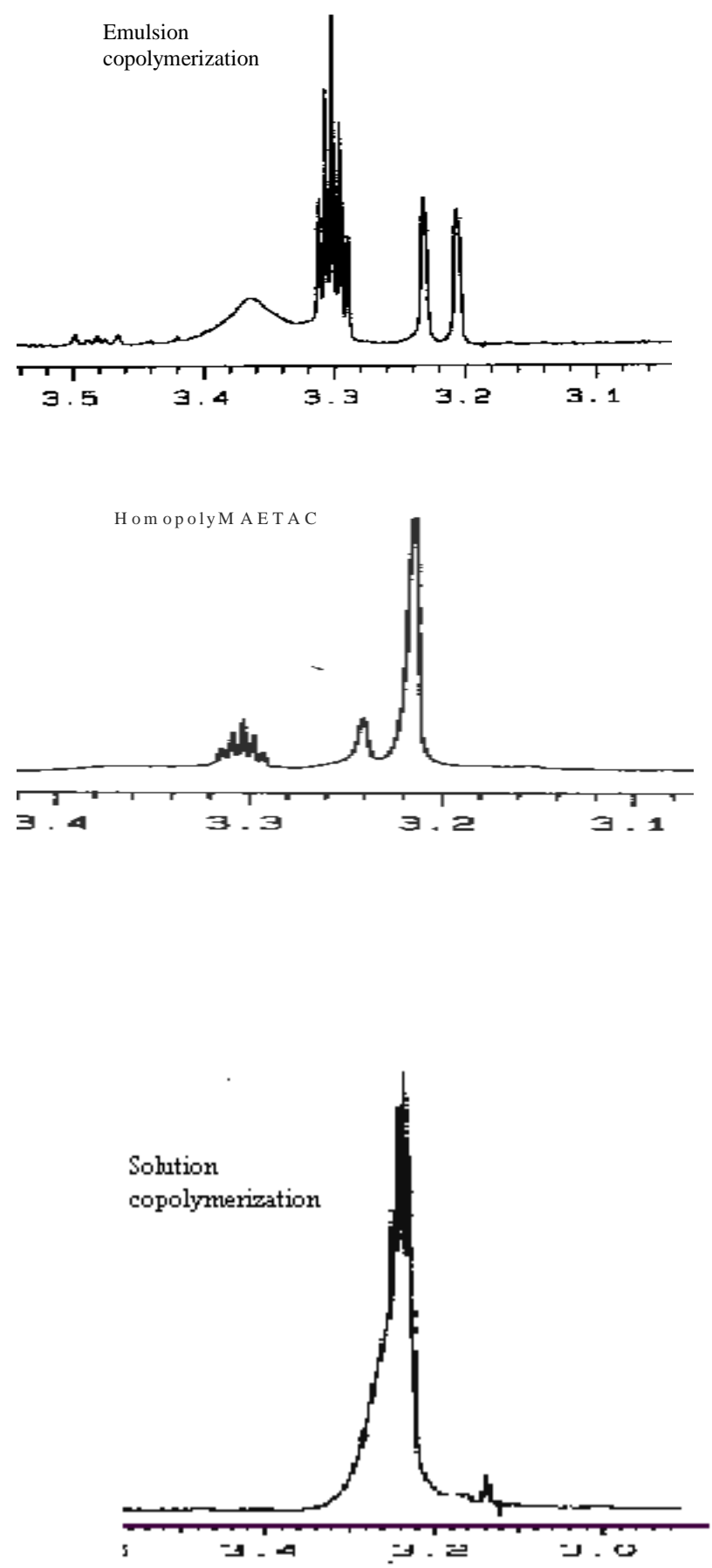

Figure 2. ${ }^{1}$ H-NMR Spectra of Emulsion Copolymerized BA/MAETAC, Homopoly(MAETAC) and Solution Copolymerized BA/MAETAC 


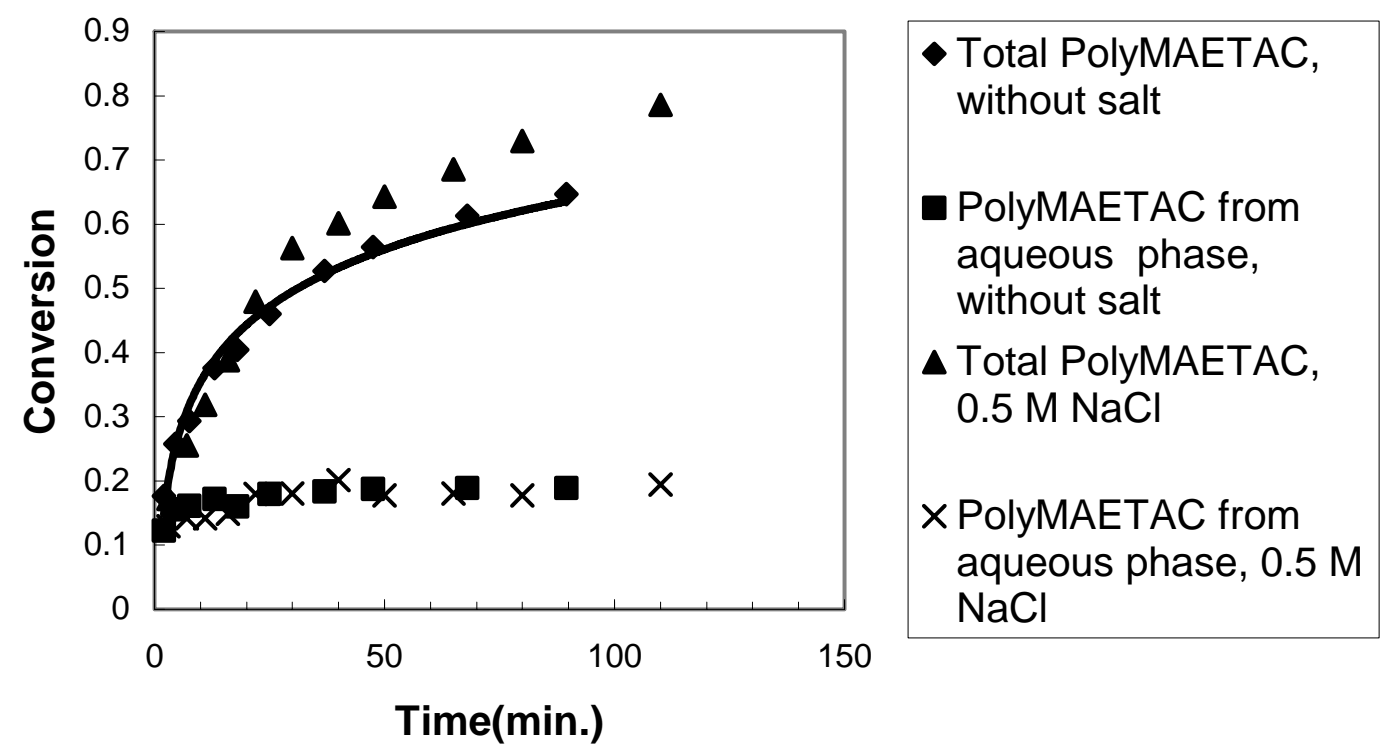

Figure 3. MAETAC Monomer Conversion, Miniemulsion Copolymerization with 13.5 wt\% MAETAC (based on total monomer) in the presence of Salt

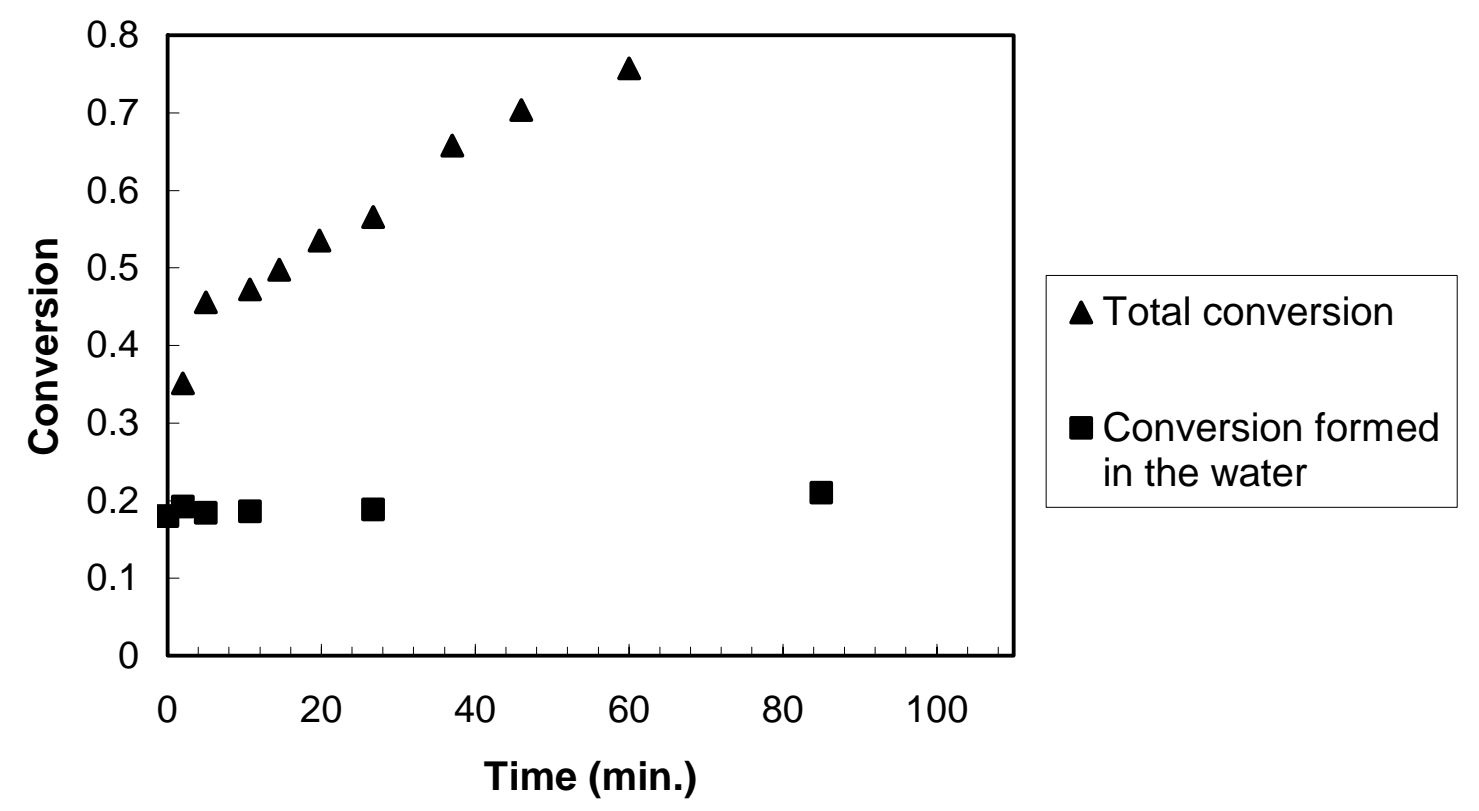

Figure 4. MAETAC Monomer Conversion, Macroemulsion Copolymerization with 13.5 wt\% MAETAC (based on total monomer) 


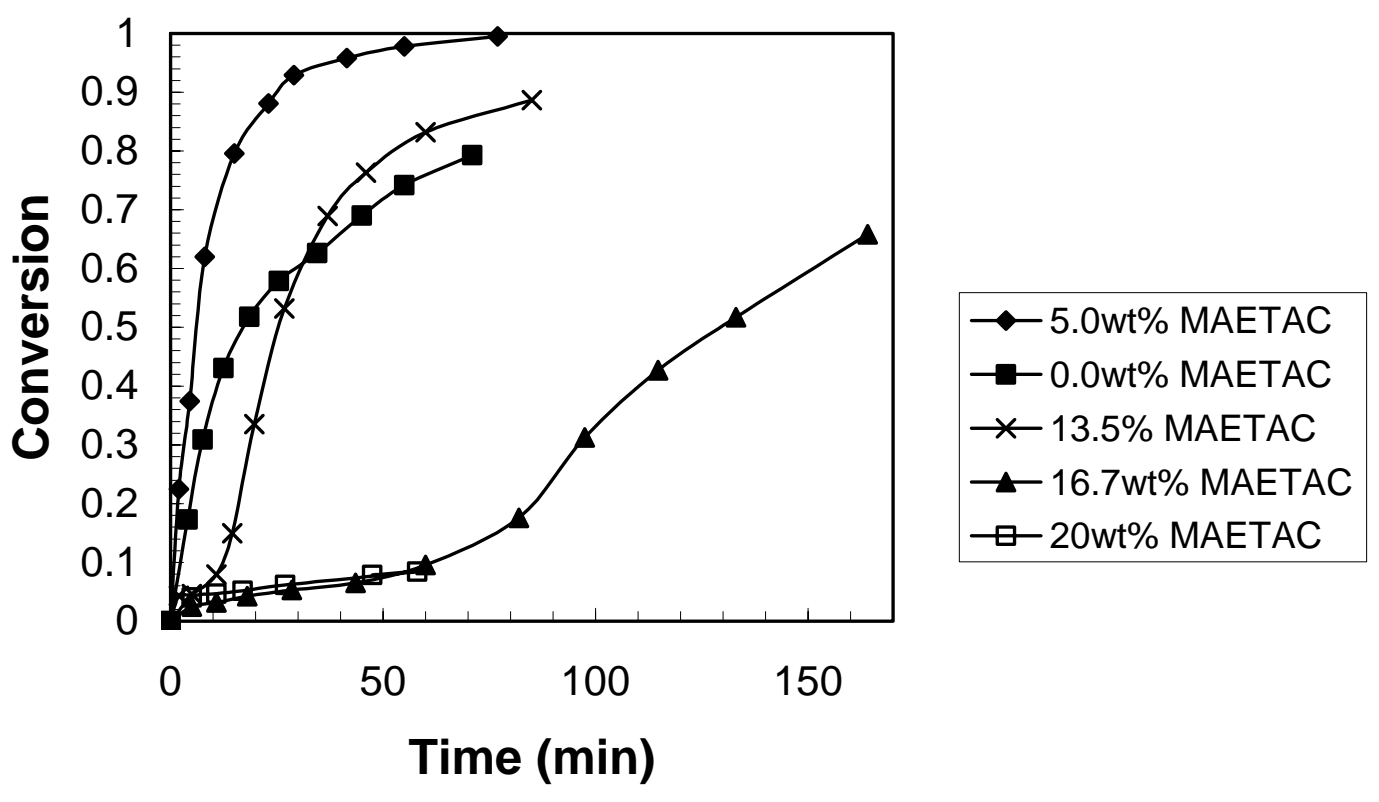

Figure 5. BA Monomer Conversion, Macroemulsion Copolymerization with Varying Levels of MAETAC (based on total monomer)

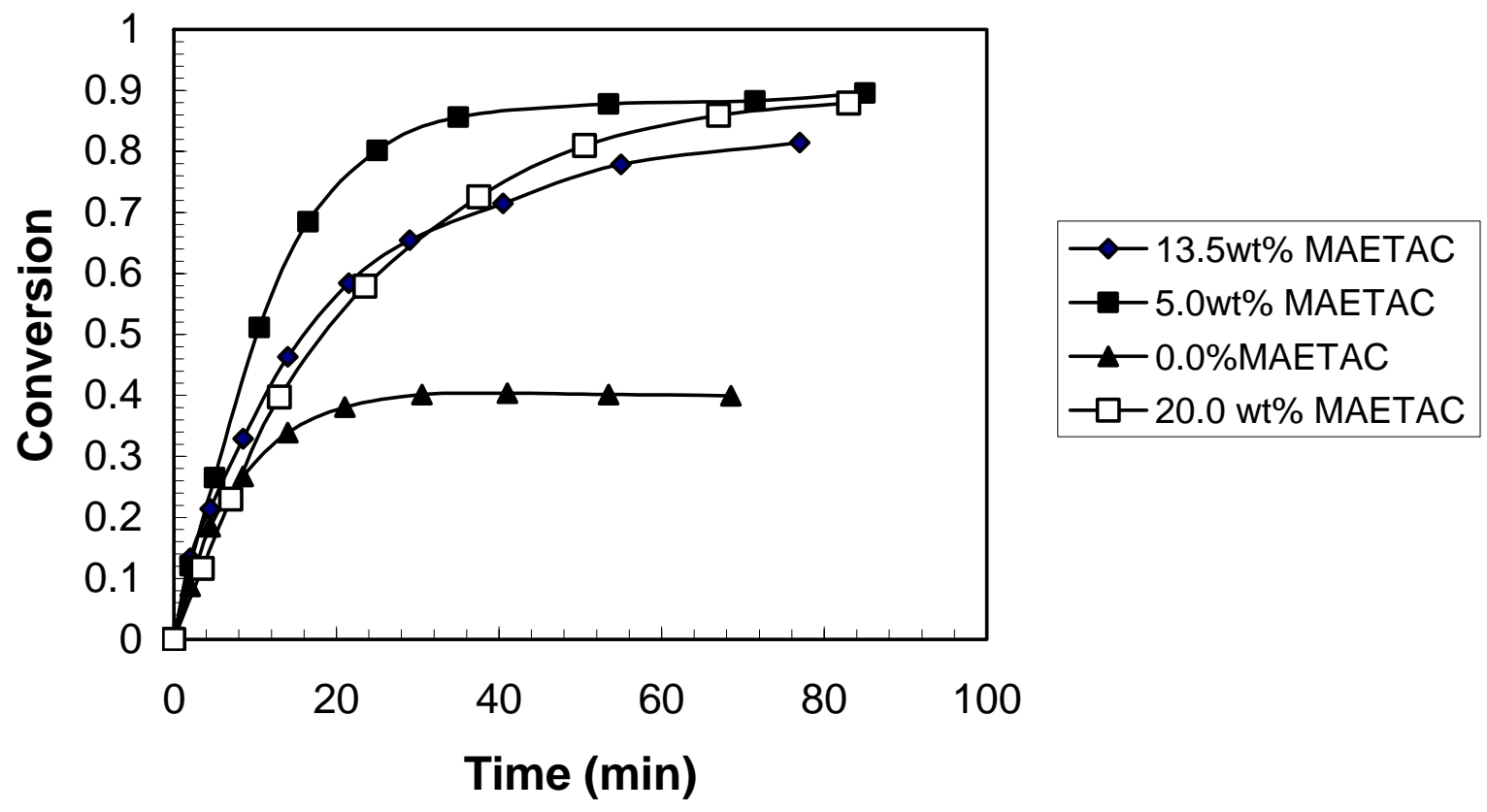

Figure 6. BA Monomer Conversion, Miniemulsion Copolymerization with Varying Levels of MAETAC (based on total monomer) 


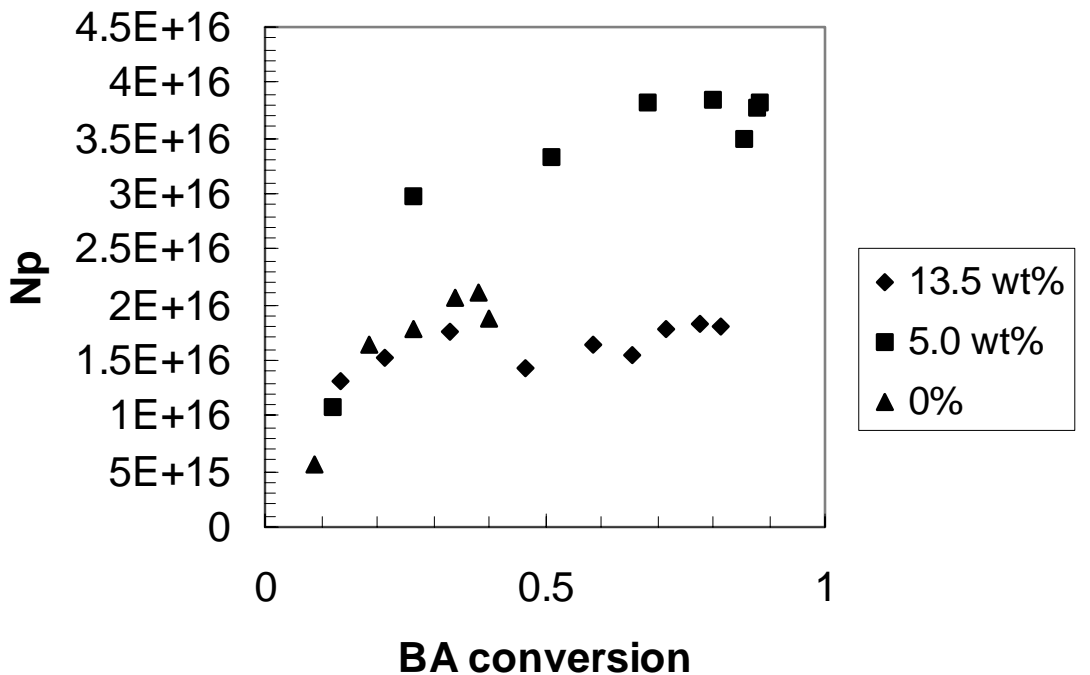

Figure 7. Particle Number Development, Miniemulsion Copolymerization with Varying Levels of MAETAC (based on total monomer)

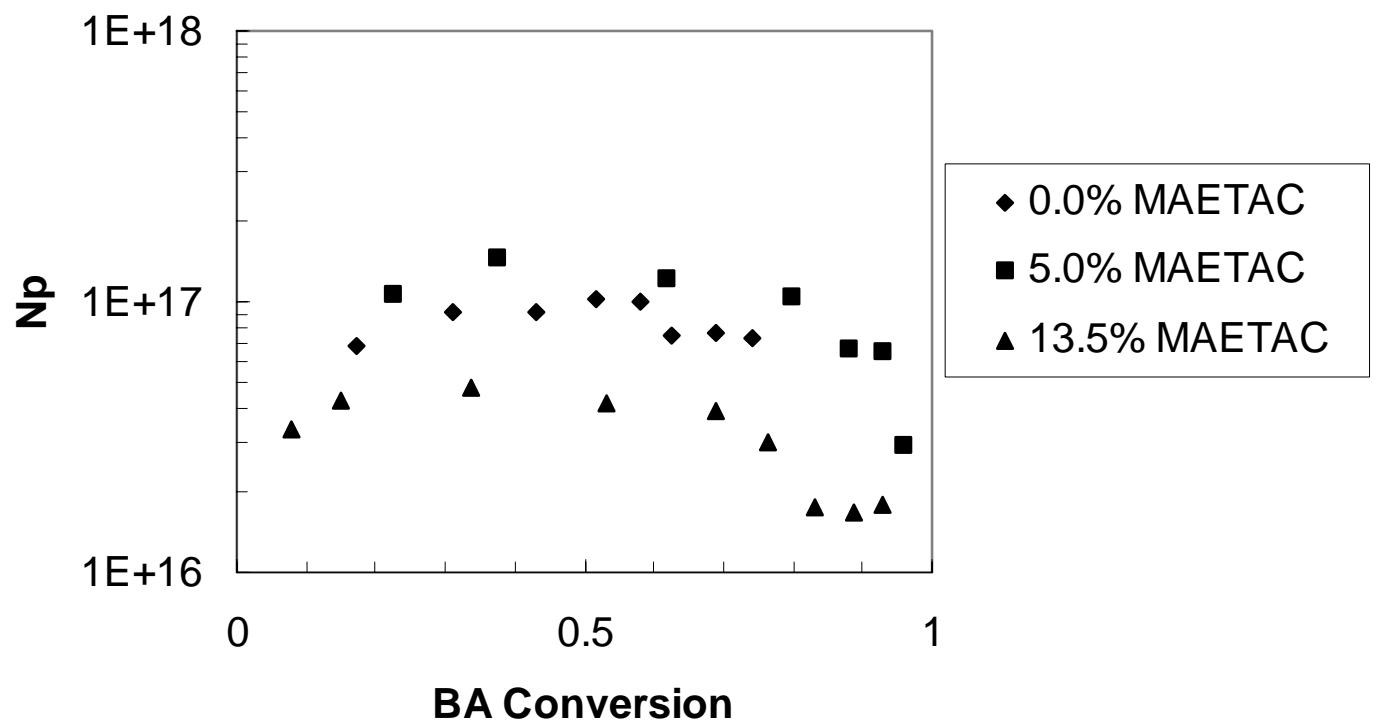

Figure 8. Particle Number Development, Macroemulsion Copolymerization with Varying Levels of MAETAC (based on total monomer) 


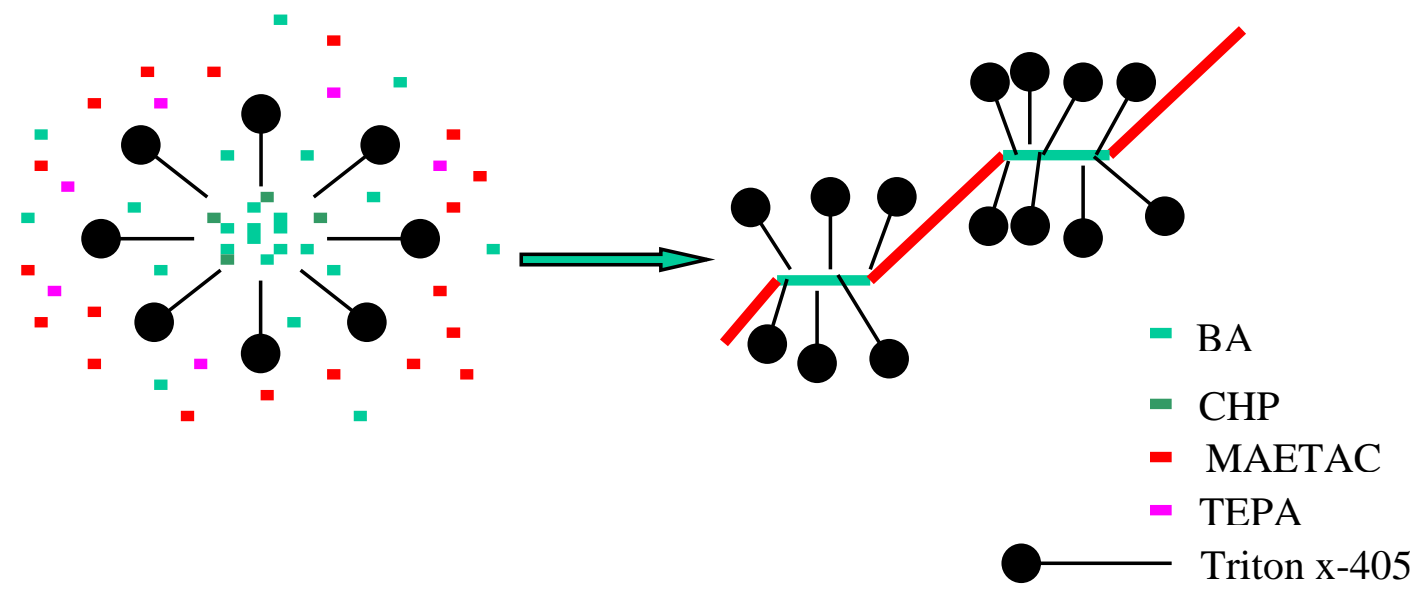

Figure 9. Schematic of the Formation of Multi-block Poly(MAETAC-BA)

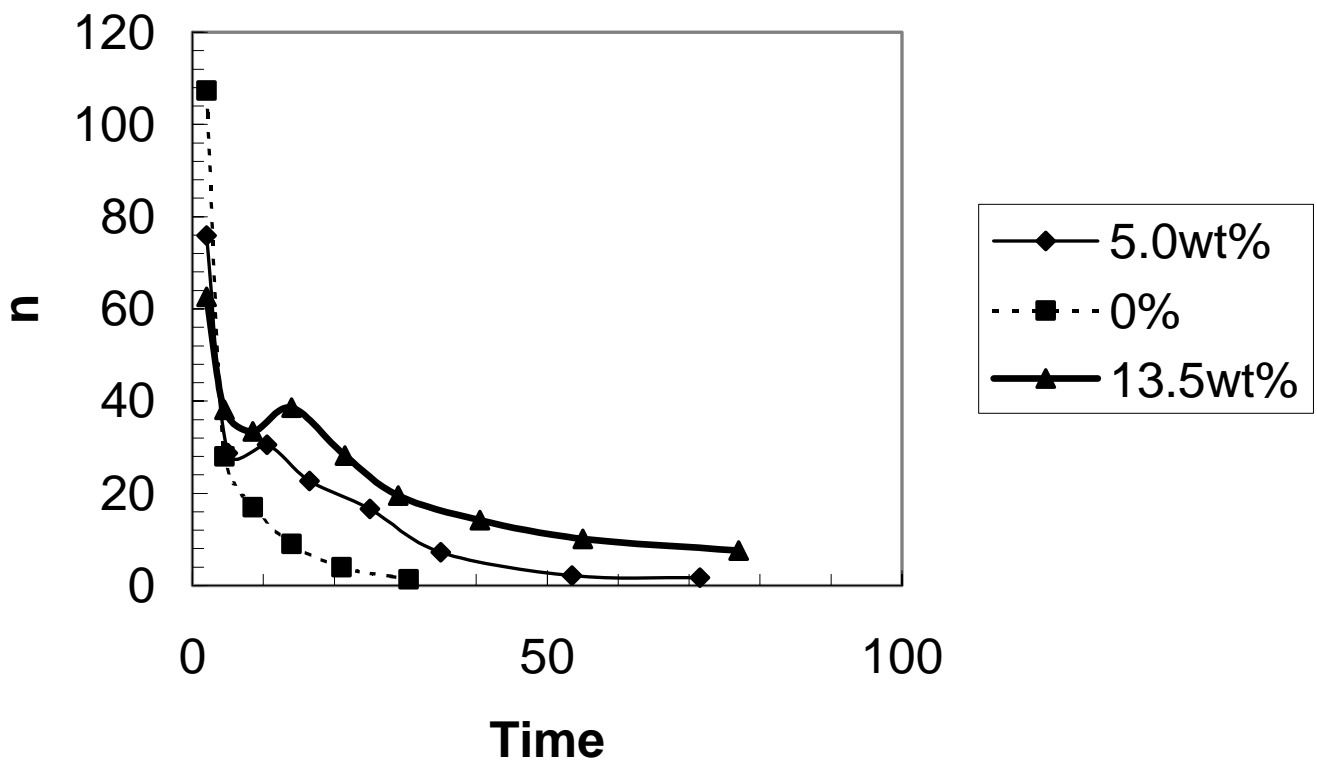

Figure 10. Number of Free Radicals per Particle, Miniemulsion Copolymerization with Varying Levels of MAETAC (based on total monomer) 


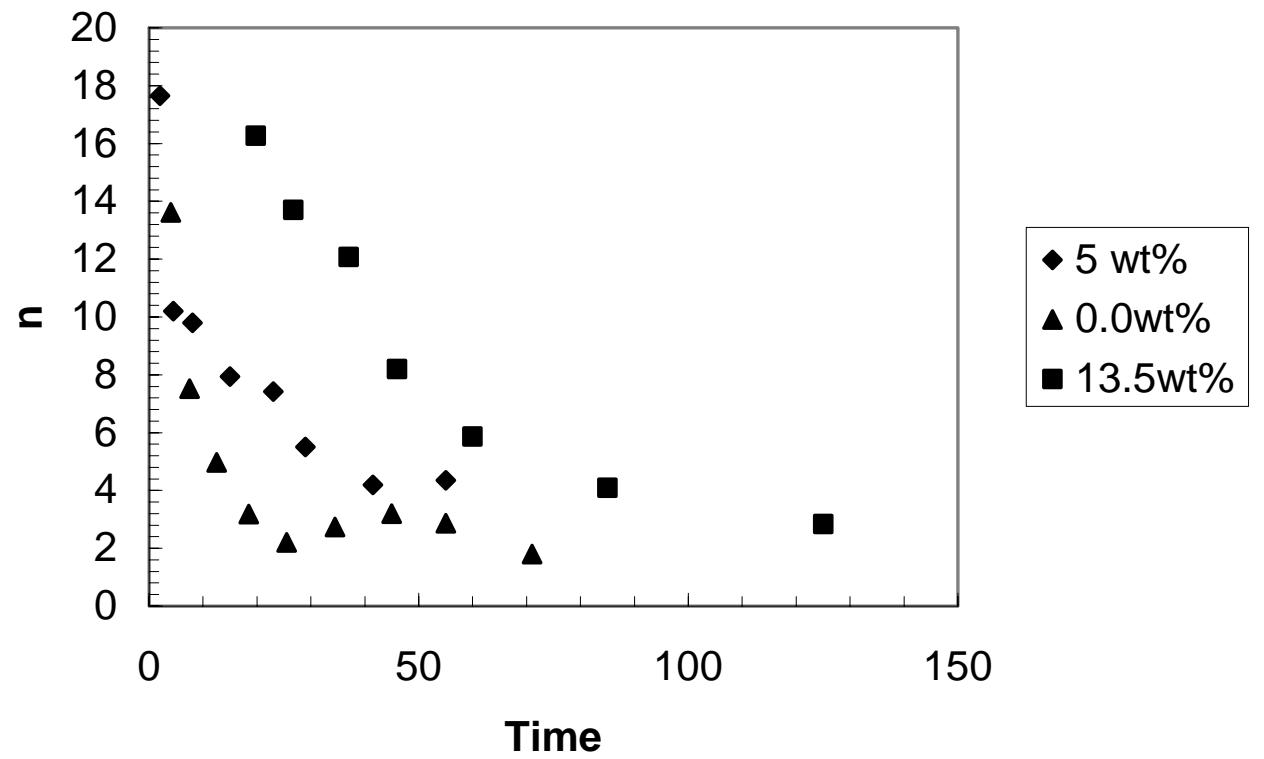

Figure 11. Number of Free Radicals per Particle, Macroemulsion Copolymerization with Varying Levels of MAETAC (based on total monomer)

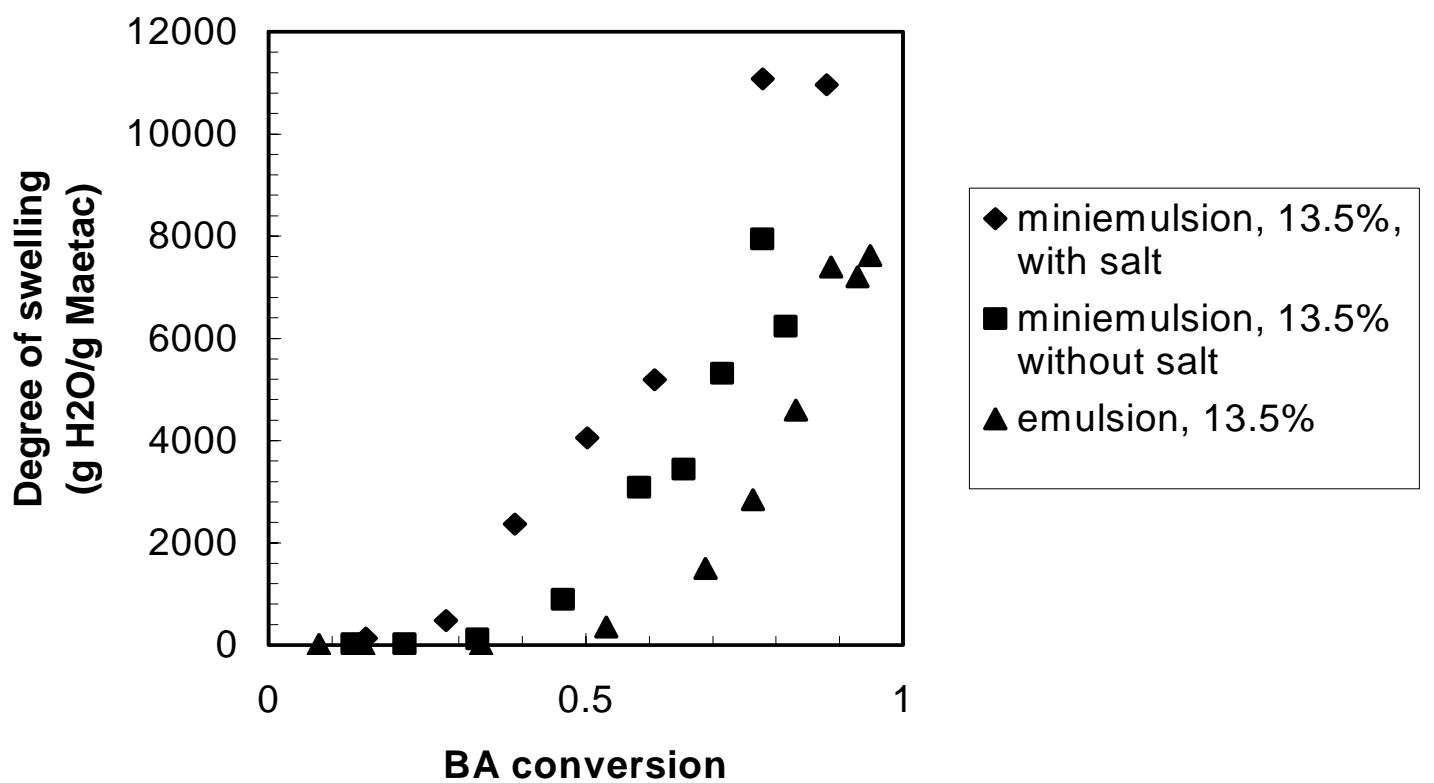

Figure 12. Degree of Swelling of the Hydration Layer as a Function of BA Conversion; 13.5 wt\% MAETAC; Macroemulsion, Miniemulsion, and Miniemulsion Polymerized 


\subsection{Water-Based Cationic PSA}

\section{$\underline{\text { Experimental }}$}

\section{Synthesis and Characterization}

Miniemulsion technique was used to synthesize water-based cationic PSA as reported above. Hexanethiol (HT) was used as chain transfer agent and ethylene glycol dimethacrylate (EGDM) was used as crosslinker to control the polymer molecular weight. The basic recipe is shown in Table 1 . Gel fraction of the polymer with crosslinker was determined by solvent extraction with Soxhlet Extractor. The dried sample was extracted by ethanol and then THF for 24 hours, respectively. Glass transition temperatures were determined on a Perkin Elmer Pyris-1 differential scanning calorimeter (DSC) under helium atmosphere. The sample was heated to $200{ }^{\circ} \mathrm{C}$, cooled to $-100{ }^{\circ} \mathrm{C}$, and then heated to $200{ }^{\circ} \mathrm{C}$ at the rate of $40{ }^{\circ} \mathrm{C} / \mathrm{min}$. PSA end use properties, its repulpability and effect on paper properties were studied as described in Part 1.

\section{$\underline{\text { Results and Discussion }}$}

\section{Polymer characterization}

The glass transition temperature of polymer was measured from DSC, and the results are shown in Figure 1. Apparently all of the copolymers with different cationic monomer feeding ratios have glass transition temperatures around $-48{ }^{\circ} \mathrm{C}$. By contrast, $\mathrm{T}_{\mathrm{g}}$ for BA hompolymer is around $-56{ }^{\circ} \mathrm{C}$. The results may indicate that the polymers in the latex from high MAETAC feeding ratio were heterogeneous.

\section{PSA properties and repulpability}

This study was intended to develop water-based water soluble/dispersible cationic PSAs, which may not cause stick problems in paper recycling. For ionic polyacrylate 
copolymer to be water soluble or dispersible, the copolymer must contain enough hydrophilic units to interact with water and then break the intermolecular bonding. However, the high cationic content may affect the end use properties of the copolymers as a pressure sensitive adhesive. The peel adhesion and shear strength of the polymers synthesized from miniemulsion polymerization are shown in Table 2. Peel adhesion is the force required to remove a PSA-coated film from a specific test surface under standard conditions (specific angle and rate). The measurement of peel adhesion involves a bonding step and a debonding or peeling step. The efficiency of the bonding process is related to the adhesive's ability to exhibit viscous flow. The debonding process involves a rapid deformation of the adhesive mass. Thus, the higher the peel strength, the higher the PSA's ability to resist bond deformation at high strain rates is. Peel strength gives a measure of adhesive or cohesive strength, depending on the mode of failure [29]. Shear resistance is measured as a force to pull the PSA material parallel to the surface to which it was affixed with a definite pressure [30] and it measures the cohesion strength of the PSA. All the copolymers (PSA1 to 3) without transfer agent showed very weak shear and peel strength. The reason may be because the molecular weight $\left(\mathrm{M}_{\mathrm{w}}\right)$ of the polymer was too high. Satas [31] reported that shear is roughly proportional to molecular weight up to relatively high $\mathrm{M}_{\mathrm{w}}$ at which the shear resistance drops off dramatically in some polymers. Peel adhesion typically exhibits a discontinuous behavior, increasing with $\mathrm{M}_{\mathrm{w}}$ up to moderate $\mathrm{M}_{\mathrm{w}}$ and then decreasing as the $\mathrm{M}_{\mathrm{w}}$ further increases. The molecular weight for water based polyacrylic PSA polymer is normally in the range of 300,000 to 1 million [32]. Generally, the molecular weight for polymer synthesized from emulsion polymerization would be about 1 million. Furthermore, because the copolymer of BA and MAETAC is heterogeneous, the MAETAC-rich portion as well as the MAETAC homopolymer in water may crystallize during drying. The crystals may restrict the molecule flow of the PSA on a substrate surface, thus significantly reduce the adhesion.

The addition of chain transfer agent (CTA) in polymerization increased the peel strength of the PSA (PSA4 and 5) significantly, but the shear adhesion was still poor. It should be noted that failure of the two PSAs with transfer agent added was cohesion failure. The results indicated the PSAs had stronger bonding with the substrate than the inter- 
molecular bonding. The reason is that the molecular weight of the PSA polymer is low. The molecular weight of the polymers with chain transfer agent can be estimated based on the Mayo equation [33]:

$$
\frac{1}{D P_{n}}=\frac{1}{P_{n, 0}}+C \frac{[C T A]}{[M]}
$$

where $\mathrm{DP}_{\mathrm{n}}$ and $\mathrm{DP}_{\mathrm{n}, 0}$ are the number average degree of polymerization with or without chain transfer agent respectively, $\mathrm{C}$ is the chain transfer constant of the CTA, [CTA] is the concentration of the chain transfer agent, and $[\mathrm{M}]$ is the concentration of the monomer. Because 1-hexanethiol as transfer agent would stay in the oil phase with butyl acrylate, the chain transfer would mostly occur with butyl acrylate. From various references [34,35], it is estimated that the chain transfer constant of 1-hexanethiol for butyl acrylate is 0.50. For PSA4, the concentration ratio of 1-hexanethiol and BA is $0.11 \%$. Thus the molecular weight of the polymer with chain transfer agent would be about 189,000 . PSA polymer with low molecular weight can flow easily and wet out the substrate better. The result is better bonding between the PSA and the substrate. But with viscous flow ability increases, the cohesion strength of the polymer decreases.

Introducing a small amount of crosslinker into the polymer can increase the polymer cohesion strength, thus increase the shear strength. The failure of the shear became adhesion failure for the crosslinked polymers. The effect of crosslinker on peel strength is not significant. However, too high crosslinker content can significantly limit the ability of polymer diffusion on a substrate, thus may reduce the peel strength.

Although from previous discussion we know the polymer latex from high MAETAC feeding ratio has core-shell structure, we had expected the water soluble shell may help the PSA film to disperse in water again. But solubility test indicated that all the polymers synthesized from miniemulsion polymerization could not re-disperse to water once they are dried. Repulping test showed that all the cationic PSAs were not repulpable, even after they formed very thin (less than $25 \mu \mathrm{m}$ thickness) PSA film on copy paper. Large sticky particles can clearly be seen from the handsheets made from the recycled pulp. The results indicated that when dry PSA film was formed from the latex emulsion, the 
water insoluble MAETAC-poor core of the latex particles coalesced together. During repulping process, the coalescent film could not be redispersed into colloidal particles.

In order to increase the redispersability of the cationic PSA, three water-soluble cationic chemicals were added to the emulsion. These water-soluble cationic chemicals include surfactant cetyltrimethylammonium bromide (CTAB), starch, and water-soluble The poly(BA-co-MAPTAC) with MAPTAC content of 15.2 mol\%. This poly(BA-coMAPTAC) polymer was made from bulk polymerization in ethanol and itself can be used as a water-soluble cationic PSA as reported in Part 1. The repulpability of the newly formulated PSA was then studied. It was found the both the CTAB and starch could not make the cationic PSAs redisperable with $4 \mathrm{wt} \%$ addition level. Furthermore, the CTAB reduced the tackiness of the PSA significantly, and the starch increased the viscosity of the PSA emulsion too much and their repulpability is reported in Table 3. However, the results indicated that water-insoluble PSA from miniemulsion polymerization became water dispersible when it was blended with a cationic PSA made from ethanol polymerization. It is also interesting that addition of certain amount of crosslinker in the PSA latex could make it repulpable. For PSA latex without crosslinker, the molecules in the latex may move easily to form an evenly coalescent layer during drying. The addition of poly(BA-co-MAPTAC) would be difficult to allow the dried PSA film to dissociate in repulping water during the paper recycling process. While for PSA latex with crosslinker, the crosslinker can hold the molecules in the latex together. The coalescence of the latexes during drying would not be even. With poly(BA-co-MAPTAC) added, the BA units, which are hydrophobic, would attach to the hydrophobic units in the PSA latex; and the MAPTAC units, which are hydrophobic, would stay outward to the aqueous phase on the latex surface. During drying, poly(BA-co-MAPTAC) would form a barrier around the latex particles as a lubricant. In the paper recycling process under high shear, the PSA would be easy to re-disperse to microparticles. The addition of a small amount of starch in some was not aimed to improve the PSA dispersability, but rather to improve the properties of paper made from the recycled paper. 
To further understand the crosslinking in the polymer latex, the gel content was measured. Ethanol was used extraction solvent to remove polymers with high MAETAC content. Tetrahydrofuran (THF) was used to remove polymers with low MAETAC content. From Table 4, it can be seen that most non-crosslinked polymer was removed by ethanol. This is not surprising because the crosslinker EDGM is hydrophobic, and thus would mainly crosslink inside the latex with butyl acrylate. The crosslinked hydrophobic core of the polymer latex would retard the coalescent during drying.

The PSA properties of the repulpable (dispersible) PSAs were tested and results are shown in Table 5. It was found that the addition of poly(BA-co-MAPTAC) have no significant effect on PSA properties.

$\underline{\text { PSA adsorption and effect on paper properties }}$

In Part 1, we have reported that the cationic PSA from solution polymerization could adsorb on fiber surfaces in paper recycling and papermaking. Here the adsorption of water based cationic PSAs made from miniemulsion polymerization is reported. Copy papers with and without 0.5 wt $\%$ of cationic PSA12 were repulped with copy paper. Pulping water was collected. After half an hour, the turbidity and charge of the supernatant of the pulping water were measured. No detectable PSA12 was found in the pulping water, which means almost all of the cationic PSA microparticles adsorbed on the fibers.

Because the cationic PSA microparticles would adsorb on fibers, and be brought into the final paper products during the papermaking process, the effect of the PSA on paper properties should be examined. Figure 2 shows the effect of cationic PSA12-15 on the paper tensile strength. It was found that the cationic PSA could slightly increase the paper strength. With starch added to the PSA, the increase of the paper strength was larger. Starch is widely used as strength agent in papermaking. The small amount of starch in the PSA should contribute to the strength improvement. Sizing is a measure of the hydrophobicity of the paper. Because the cationic PSA is partly hydrophobic, it may 
have some effect on the paper sizing properties. The sizing effect of PSA was measure by Hercules Sizing Test (HST). It was found that with $0.5 \%$ addition level of cationic PSA in the paper, the HST increased from to second to a few seconds. This effect is negligible.

\subsubsection{Conclusions}

Water based cationic PSAs with various compositions were synthesized from miniemulsion polymerization. It was found the PSA latex with high cationic monomer MAETAC feeding ratio was very heterogeneous. The cationic PSA itself could not be dispersed in paper recycling process. However, when the cationic PSA was formulated with another cationic hydrophobic water-soluble polymer, the PSA was fully dispersible, and would not form stickies during repulping and papermaking processes. The dispersed PSA could be easily removed from the papermaking water system by adsorbing onto negatively charged fiber surfaces. Furthermore, the adsorbed colloidal PSA could improve the paper strength of the final paper product. Thus, water based cationic dispersible PSAs, which are friendly for paper recycling, were successfully developed. 
Table 1. Basic Recipe

\begin{tabular}{lll}
\hline Name & Formula & Amount (g) \\
\hline Butyl acrylate & $\mathrm{H}_{2} \mathrm{C}=\mathrm{CHCO}_{2}\left(\mathrm{CH}_{2}\right)_{3} \mathrm{CH}_{3}$ & 75 \\
MAETAC & $\mathrm{H}_{2} \mathrm{C}=\mathrm{C}\left(\mathrm{CH}_{3}\right) \mathrm{CO}_{2}\left(\mathrm{CH}_{2}\right)_{2} \mathrm{~N}\left(\mathrm{CH}_{3}\right)_{3} \mathrm{Cl}$ & Variable \\
Triton X-405 & $4-\left(\mathrm{C}_{8} \mathrm{H}_{17}\right) \mathrm{C}_{6} \mathrm{H}_{4}-\left(\mathrm{OCH}_{2} \mathrm{CH}_{2}\right)_{\mathrm{n}} \mathrm{OH}, \mathrm{n}=40$ & 6.43 \\
CHP & $\mathrm{C}_{6} \mathrm{H}_{5} \mathrm{C}\left(\mathrm{CH}_{3}\right)_{2} \mathrm{OOH}$ & 1.0 \\
TEPA & $\mathrm{HN}\left(\mathrm{CH}_{2} \mathrm{CH}_{2} \mathrm{NHCH}_{2} \mathrm{CH}_{2} \mathrm{NH}_{2}\right)_{2}$ & 1.0 \\
water & $\mathrm{H}_{2} \mathrm{O}$ & 115 \\
EGDM & {$\left[\mathrm{H}_{2} \mathrm{C}=\mathrm{C}_{\left.\left(\mathrm{CH}_{3}\right) \mathrm{CO}_{2} \mathrm{CH}_{2}\right]_{2}}\right.$} & Variable \\
1-Hexanethiol & $\mathrm{CH}_{3}\left(\mathrm{CH}_{2}\right)_{5} \mathrm{SH}_{2}$ & Variable \\
Hexadecane & $\mathrm{CH}_{3}\left(\mathrm{CH}_{2}\right)_{14} \mathrm{CH}_{3}$ & 1.5 \\
\hline
\end{tabular}

Table 2. Adhesive properties of cationic PSAs.

\begin{tabular}{|c|c|c|c|c|c|c|c|}
\hline \multirow[t]{2}{*}{ Sample } & \multicolumn{3}{|c|}{$\begin{array}{l}\text { Feeding composition for } \\
\text { variables (mol\%) }\end{array}$} & \multicolumn{2}{|c|}{$\begin{array}{l}180^{\circ} \text { Peel Adhesion } \\
(10 \text { min Dwell) (PSTC-1) }\end{array}$} & \multicolumn{2}{|c|}{$\begin{array}{l}\text { Shear Strength } \\
\text { (PTSC-7) }\end{array}$} \\
\hline & MAETAC & HT & EGDM & g/in & $\begin{array}{l}\text { Failure } \\
\text { Type* }\end{array}$ & hour & $\begin{array}{l}\text { Failure } \\
\text { Type }\end{array}$ \\
\hline PSA1 & 8.4 & 0 & 0 & 40.8 & $\mathrm{~A}$ & 0 & $\mathrm{~A}$ \\
\hline PSA2 & 10.9 & 0 & 0 & 25.3 & A & 0 & A \\
\hline PSA3 & 16.0 & 0 & 0 & 10.6 & $\mathrm{~A}$ & 0 & $\mathrm{~A}$ \\
\hline PSA4 & 10.9 & 0.1 & 0 & 560.5 & $\mathrm{C}$ & 0.1 & $\mathrm{C}$ \\
\hline PSA5 & 10.9 & 0.14 & 0 & 425.3 & $\mathrm{C}$ & 0.1 & $\mathrm{C}$ \\
\hline PSA6 & 10.9 & 0.1 & 0.18 & 339.7 & $\mathrm{~A}$ & 6.8 & $\mathrm{~A}$ \\
\hline PSA7 & 10.9 & 0.14 & 0.18 & 456.8 & $\mathrm{~A}$ & 11.7 & A \\
\hline PSA8 & 10.9 & 0.14 & 0.36 & 234.2 & $\mathrm{~A}$ & 6.0 & $\mathrm{~A}$ \\
\hline PSA9 & 16.0 & 0.13 & 0.34 & 180.3 & $\mathrm{~A}$ & 0.3 & $\mathrm{~A}$ \\
\hline
\end{tabular}

* A: Adhesion failure; C: Cohesion failure. 
Table 3. The repulpability of formulated cationic PSAs.

\begin{tabular}{|l|l|l|l|l|}
\hline \multirow{2}{*}{ PSA } & \multicolumn{2}{l|}{ PSA composition (wt\%) } & \multirow{2}{*}{ Repulpability } \\
\cline { 2 - 5 } & Emulsion & BAM & Starch & \\
\hline PSA10 & 96 (PSA4) & 4 & 0 & No \\
\hline PSA11 & 96 (PSA5) & 4 & 0 & No \\
\hline PSA12 & 96 (PSA6) & 4 & 0 & Repulpable \\
\hline PSA13 & 96 (PSA6) & 3 & 1 & Repulpable \\
\hline PSA14 & 96 (PSA7) & 4 & 0 & Repulpable \\
\hline PSA15 & 96 (PSA7) & 3 & 1 & Repulpable \\
\hline
\end{tabular}

Table 4. Gel content in the crosslinked polymers.

\begin{tabular}{|l|l|l|l|l|}
\hline Sample & \multirow{2}{*}{$\begin{array}{l}\text { HT } \\
(\mathrm{mol} \%)\end{array}$} & \multirow{2}{*}{$\begin{array}{l}\text { EGDM } \\
(\mathrm{mol} \%)\end{array}$} & \multicolumn{2}{|l|}{ Gel content after solvent extraction } \\
\cline { 5 - 5 } & 0.11 & 0.2 & Ethanol & Ethanol and THF \\
\hline PSA6 & 0.16 & 0.2 & 0.736 & 0.671 \\
\hline PSA7 & 0.16 & 0.769 & 0.648 \\
\hline PSA8 & 0.16 & 0.4 & 0.702 \\
\hline
\end{tabular}

Table 5. Adhesive properties of water dispersible cationic PSAs.

\begin{tabular}{|l|l|l|l|l|}
\hline \multirow{2}{*}{ Sample } & \multicolumn{2}{|l|}{$\begin{array}{l}180^{\circ} \text { Peel Adhesion } \\
(10 \text { min Dwell })(\text { PSTC- })\end{array}$} & \multicolumn{2}{l|}{ Shear Strength (PTSC-7) } \\
\cline { 2 - 5 } & g/in & Failure Type & hour & Failure Type \\
\hline PSA6 & 339.7 & A & 6.8 & A \\
\hline PSA12 & 376.8 & A & 10.3 & A \\
\hline PSA13 & 3542 & A & 7.7 & A \\
\hline PSA7 & 456.8 & A & 11.7 & A \\
\hline PSA14 & 480.3 & A & 15.3 & A \\
\hline PSA15 & 463.5 & A & 12.6 & A \\
\hline
\end{tabular}




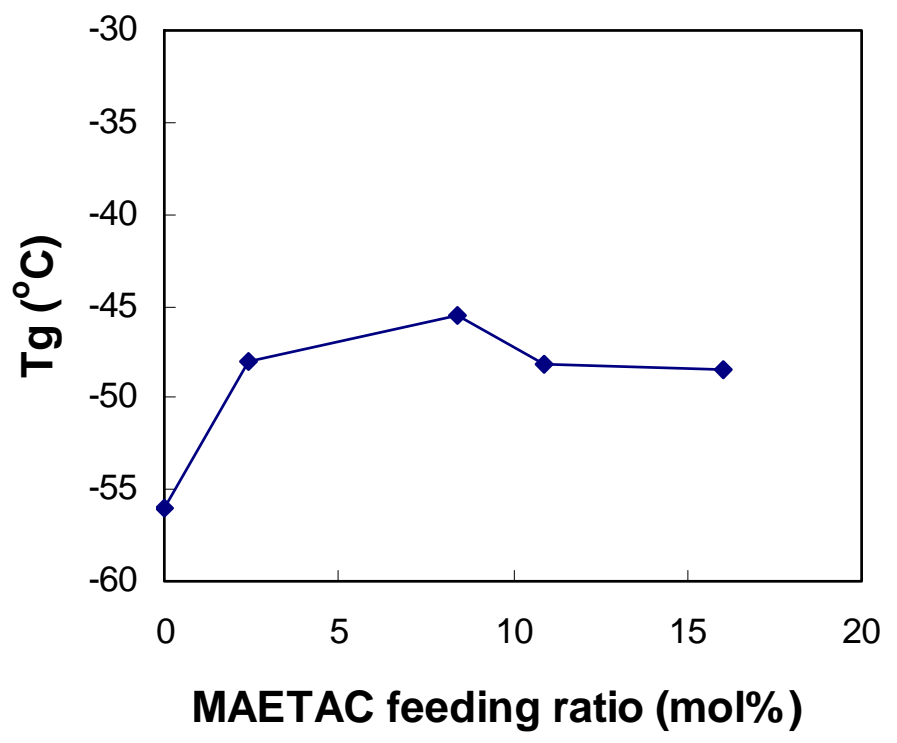

Figure 1. The glass transition temperature (Tg) for polymers with varying level of MAETAC (in feeding).

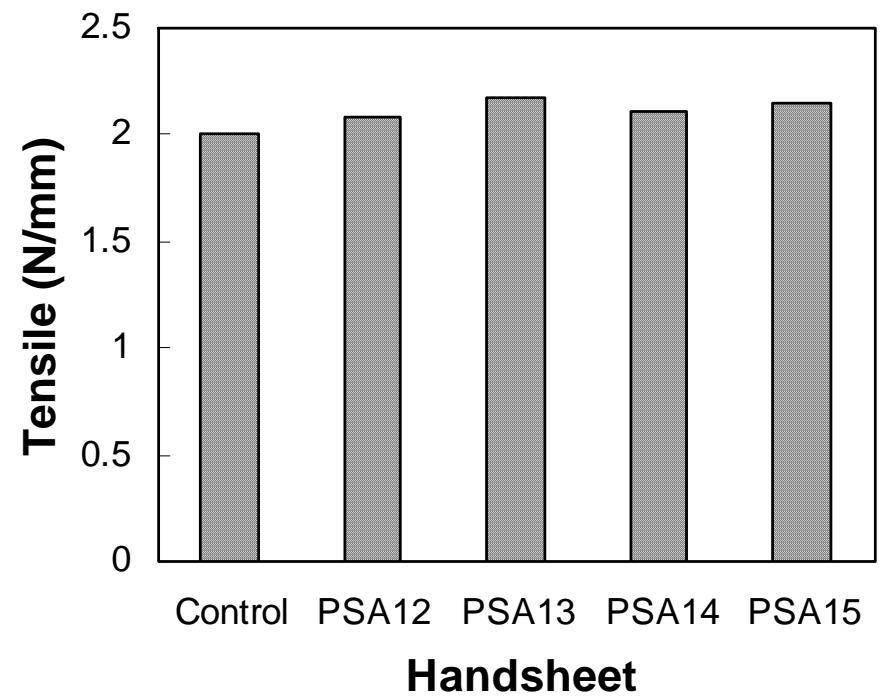

Figure 2. The effect of cationic PSAs on the paper tensile strength. PSA content in the paper handsheet is $0.5 \%$. 


\section{References}

1. R.G. Gilbert, J.F. Anstey, N. Subramaniam and M.J. Monteiro, Polymer Preprints, 40(2), 297 (1999).

2. B. Siadat, Ph.D. Thesis, Dept. Chem. Eng., Univ. of Massachusetts, 1979.

3. S.R. Turner, R.A. Weiss and R.D. Lundberg, J. Polym. Sci., Polym. Chem. Ed. 37, 535 (1985).

4. X.Q. Wu and F. J. Schork, Kinetics of Miniemulsion Polymerization of Vinyl Acetate with Nonionic and Anionic Surfactants, J. Appl. Polym. Sci., 81(7), 1691 (2001).

5. R.G. Gilbert, "Emulsion Polymerization: A Mechanistic Approach", Academic Press, New York, 1995.

6. E. Ozdeger, E.D. Sudol, M.S. El-Aasser and A.J. Klein, J. Polym Sci. Part A, 35, 3827 (1997)..

7. P. Mallya and S.S. Plamthottam, Termination Rate Constant in Butyl Acrylate Batch Emulsion Polymerization. Polym Bull (Berlin) 21 (5), 497 (1989).

8. M.T. Charreyre, V. Razafindrakoto, L. Veron, T. Delair, and C. Pichot, Macromol. Chem. Phys., 195, 2141 (1994).

9. H.Y. Parker, D.G. Westmoreland and H.R. Chang, Macromol., 29, 5119 (1996).

10.D. Kukulji and R.G. Gilbert, Polymerization at High Conversion. In Polymeric Dispersions. Principles and Applications; J. M. Asua (Eds.)., Kluwer Academic, Dordrecht, pp 97 (1997).

11. S. Numo-Donlunas, A.I. Rhoton, S. Corona-Galvan, J. E. Puig., and E.W. Kaler, Polymer Bulletin, 30, 207 (1993).

12. L.F. Antonova, G. V. Leplyanin, Ye. Ye. Zayev, and S. R. Rafikov, Polymer Sci. U.S.S.R., 20, 778 (1978).

13. J. H. Kim, M. Chainey, M. S. El Aasser and J. W. VanderHoff, J. Polym. Sci., Polym. Chem. Ed., 30, 535 (1992).

14. B. Emelie, D. Pichot, and Guillot, J. Makromo. Chem., 189, 1879 (1988).

15. G. L. Shoaf and G. W. Poehlein, Polymer Reaction Eng., 93, 1 (1992).

16. D. Charmot, J. F. D’Allest and F. Dobler, Polymer, 37, 5237 (1996). 
17. F. Ganachaud, F. Sauzedde, A. Elaissari, C. Pichot, J. Appl. Polym. Sci., 65, 2315 (1997).

18. J. H. Kim, M. Chainey, M. S. El Aasser and J. W. VanderHoff, J. Polym. Sci., Polym. Chem. Ed., 28, 3188 (1990).

19. N. Nomura, J Ikoma, and K. Fujita, in Polymer Latex, E.S. Daniels, E. D. Sudol, and M. S. El-Aasser (Eds). ACS 492, 55, Washington DC 1992.

20. J. A. Alduncin, J. Forcada, M. J. Barandiaran, and J. M. Asua, J. Polym. Sci. Part A, 29, 1265 (1991).

21. J. M. H. Kusters, D. H. Napper, R. G. Gilbert, A. L. German, Macromol., 25, 7043 (1992).

22. D. Colombie, E. D. Sudol, and M. S. El-Aasser, Macromol., 33, 4347 (2000)

23. T. J. Tulig and M. Tirrell, Macromol., 14, 1501 (1981).

24. D. C. Blackley, Emulsion Polymerization, John Wiley \& Sons, New York and Toronto, 1975, p. 244.

25. Y. Luo, F. J. Schork, Y. Deng, and Z. Yan, Polymer Rect. Eng., 9(3), 183 (2001).

26. F. Candau, Adv. Colloid Interface Sci., 79 (2-3), 149 (1999).

27. R. S. Harland and R. K. Prud'homme, Polyelctrolyte Gels, ACS 480, Washington DC 1992.

28. S. Corona-Galvan, J. Castaneda-Perez, A. Martines-Gomez, J. E. Puig and P. C. Schulz, Colloid Polym. Sci., 268, 778 (1990)

29. P. Caton, European Adhesives and Sealants, 12, 18 (1990).

30. I. Benedek and L.J. Heymans, Pressure Sensitive Adhesive Technology, Marcel Dekker, Inc, New York, 214 (1997).

31. D. Satas, Handbook of Pressure Sensitive Technology, Van Nostrand-Rheinhold Co., New York, 1982.

32. I. Benedek and L.J. Heymans, Pressure Sensitive Adhesive Technology, Marcel Dekker, Inc, New York, 80 (1997).

33. F.R. Mayo, J. Am. Chem. Soc., 65, 2324 (1943).

34. E.H. Riddle, "Monomeric Acrylic Esters", Reinhold Publishing Corp., New York, 57 (1954).

35. J.L. Fuente and E.L. Madruga, J. Polym. Sci. Polym. Chem., 36, 2913 (1998). 


\section{PART 3. REMOVAL OF CONTAMINANTS FROM PAPERMAKING SYSTEM}

Pulps, especially recycled pulps contain large amount of negatively charged dissolved and colloidal contaminants. These contaminants are often negatively charged, and are commonly referred as "anionic trash". Some contaminants are tacky in nature, and they are referred as microstickies. Although much research about dissolved and colloidal contaminants has been done in the past 20 years, some questions remain unanswered and many conflicting results have been reported. Application of suitable cationic polyelectrolytes that could precipitate detrimental substances, including microstickies, onto fines or fiber in an early stage of the papermaking process has been reported [1,2]. On the other hand, Wagberg and Odberg indicated there is no fixation of anionic substances by addition of cationic charge neutralizer in their early study [3], but they pointed out that the anionic trash can be fixed to fiber surface if the concentration of anionic substances or the consistency of fibers is high. Fundamentally, people still don't understand if the contaminants can be effectively removed by the fixation process.

Since fixation has the benefit to avoid buidup of stickies in the whitewater system, it is worth to investigate its effectiveness under various papermaking conditions. We have conducted studies to answer to following questions:

1) Can the anionic trash be fixed to fiber surfaces?

2) What is the effect of papermaking conditions, including $\mathrm{pH}$, shear force, fixative agents, flocculants, etc, on fixation?

3) Is the fixation a good method for controlling anionic trash?

4) How does the "fixed" anionic trash affect the paper properties?

The most effective method to control microstickies in current paper recycling mills is to use chemicals, such as cationic polymer, talc, surfactant etc. to modify the surface chemistry and reduce the tackiness of the microstickies. These methods are commonly called "sticky control". However, it is obvious that "sticky control" can only temporally solve stickies deposition problem, but not remove them. Thus it may be still desirable to 
remove the microstickies from the papermaking system. We have explored several methods in removing microstickies. One method is to use flotation to remove microstickies from the recycling pulp. Although past works have been done in using flotation to remove stickies, those works were aim at total stickies and the removal efficiency were all found to be less than 60-70\% [4-6]. No study has been conducted specifically for the removal of microstickies. When cationic water soluble/dispersible PSAs are introduced into papermaking system, they may affect the charge balance in papermaking. Thus it would be interest to know how cationic polymers would affect the control of contaminants in papermaking system. In this study, we used poly(diallyldimethylammonium chloride) (polyDADMAC) as model cationic polymer instead of cationic PSA.

\subsection{Removal of Contaminants through Fixation}

\section{Experimental}

\section{Materials}

Bleached kraft pulp was used in the experiments. The kraft pulp lap was first soaked in water overnight and then refined in a Valley beater to a freeness of $450 \mathrm{ml}$. The pulp was washed in a Bauer-McNett fiber classifier with 150-mesh screen to remove the fines and contaminants. The retained fibers were collected and stored in a cool room at about $10 \%$ consistency for later use.

Lignosulfonic acid sodium salt (Aldrich) was used as model water-soluble anionic contaminants. Polyacrylate based pressure-sensitive adhesive emulsion (B.F. Goodrich) was used as model microstickies. Poly(diallyldimethylammonium chloride) (polyDADMAC, Aldrich) with $\mathrm{Mw}$ of $100 \mathrm{~K}-200 \mathrm{k}$ and $400 \mathrm{~K}-500 \mathrm{~K}$, and polyethyleneimine (PEI, BASF Corp) were used as cationic coagulants. Cationic polyacrylamide (CPAM) Percol 175 (Ciba Specialty), anionic polyacrylamide (APAM) 
N7520 (Nalco), and polyethylene oxide (PEO, Mw 8 million, Aldrich) / Phenolic formaldehyde Resin (PFR, Boredon Co.) were used as flocculants.

\section{Fixation of microstickies and lignin}

Samples of pulp suspension at a consistency of $0.6 \%$ with $200 \mathrm{ppm}$ microstickies and 100 ppm lignin were placed in a DDJ Jar with 200 mess screen and agitated at certain speed. A prescribed amount of polyDADMAC or PEI was added and agitated for another 10 minutes. If flocculant was added afterwards, the mixture was agitated for further 30 seconds. The mixture was then dewatered. The first $20 \mathrm{ml}$ of filtrate was discarded and the following $100 \mathrm{ml}$ was collected. The zeta potential of the filtrate was analyzed by Malvern 3000 zeta sizer. Lignin concentration in the filtrate was determined by measuring the absorbance at $280 \mathrm{~nm}$ using a HP8453A UV-VIS Spectrophotometer. Before the UV measurement, the sample was first treated using concentrated sulfuric acid to dissolve the complex particles that were formed by two opposite charged polymers. Total organic carbon was determined with a Shimadzu 5050 TOC analyzer in order to determine the microsticky concentration.

For the Scanning Electronic Microscopy (SEM0 analysis, model process water was used. The model process water was prepared from pulping office white papers, which contain $1 \%$ of PSA (PSA was coated on office white paper with Hand roller) and 1\% coated materials (coating broke from Mead Corp with 15\% coated material). The pulping conditions are the same as that for bleached kraft pulp lap. The process water was collected by screening the pulp through a 200 mess screen. The filtrate was then collected and left in a cool room for 2 days. The small fines and filler materials passed through the screen and settled to the bottom of the container during this setting period. The supernatant, containing dissolved and colloidal contaminants, was collected for test.

Effect of microsticky and lignin fixation on paper physical properties 
Bleached kraft pulp at the fiber consistency of $0.3 \%$ with certain amount of microstickies and lignin was used for making the hand sheets. A prescribed amount of polyDADMAC was then added. Paper sheets were then made on a British Handsheet Mold according to TAPPI 205 om-88. The sheets made were conditioned for 48 hours and physical properties were measured according to TAPPI standards.

\section{$\underline{\text { Results and discussion }}$}

The effect of fixation agents in the presence of flocculant

Currently in the paper industry, polyDADMAC and PEI are the most widely used cationic polymeric neutralizers to control charge in papermaking. It would e interest to know their effect on the fixation of microstickies and water-soluble contaminants. Figure 1 shows the lignin and microsticky concentrations, the zeta potential of the residue in filtrate as a function of the fixation agent polyDADMAC with $4 \mathrm{ppm}$ flocculant CPAM added. The CPAM was added in order to improve the fixation and simulate the mill conditions. Figure 2 shows the same results but used PEI as fixation agent instead of polyDADMAC. When polyDADMAC was used as fixation agent with flocculant CPAM, maximum fixation occurred at near zero charge point. Up to $90 \%$ of microstickies and $70 \%$ of lignin could be fixed. But the polyDADMAC was only effective in a narrow concentration range from 25 to $40 \mathrm{ppm}$. When the dose of polyDADMAC was increased further to reverse the surface charge of the residue, the amount of fixed microstickies and lignin began to decrease. This is because microstickies and aggregated lignin were restabilized to form cationic charged microparticles.

PEI also fixed over $90 \%$ of microstickies and $70 \%$ of lignin in the presence of cationic flocculant CPAM. It was effective even when the surface charge of the residue was reversed. PEI could be effective in a very broad concentration range from $60 \mathrm{ppm}$ to over $120 \mathrm{ppm}$. This may be mainly due to the weaker charge of PEI at high $\mathrm{pH}$ (pH8). Even when the microparticles absorbed cationic PEI and changed to cationic charge, the 
repulsion force between particles and fibers was still weak, and thus flocculation of CPAM could still be very effective.

The effect of polyDADMAC molecular weight on fixation

Mills use cationic charge neutralizes from different sources with different molecular weights. It is worth to know if the molecular weight will have any significant effect on fixation. Figure 3 shows the effect of polyDADMAC molecular weight on fixation when used with cationic flocculant CPAM. It was found that the molecular weight has no obvious effect on fixation. The maximum fixation efficiency was the same. The reason may be that polyDADMAC with Mw 100,000 - 200,000 and Mw 400,000-500,000 still have similar charge neutralization effect. With molecular weight in that range, the flocculant effect would be insignificant.

The effect of $\mathrm{pH}$ on fixation

Different mills operate in different $\mathrm{pH}$. Obviously the $\mathrm{pH}$ effect on fixation efficiency for different fixation agents should be evaluated. Figures 4 and 5 show the fixation efficiency with polyDADMAC and PEI as fixative agents respectively and with CPAM as flocculant at $\mathrm{pH} 5$. Comparing them with the results obtained at $\mathrm{pH} 8$ which are shown in Figures 1 and 2, it was found that $\mathrm{pH}$ does not have effect on the effectiveness of polyDADMAC, but has significant effect on the effectiveness of PEI. It is known that the charge density of PEI strongly depends on the $\mathrm{pH}$. At lower $\mathrm{pH}$, PEI has higher charge density. Thus less PEI is needed for best fixation at low $\mathrm{pH}$. Generally the fixation efficiency is lower for PEI at $\mathrm{pH} 5$ than that at $\mathrm{pH}$ 8. Further research is needed to understand this.

The effect of shear force on fixation

During papermaking, pulps are constantly subjected to high shear. Figure 6 shows the effect of shear force on the fixation with different flocculants. Apparently higher shear 
force reduces the fixation efficiency. Flocculant has significant effect on fixation. PEO/PFR has more shear tolerance than CPAM. The results may indicate that retention play an important role in fixation.

\section{The effect of flocculant on fixation}

Previous results have shown that although the right dose of fixative agent is needed for better fixation, flocculant may have also played a critical role. Figure 7 shows the effective of fixation with or without flocculants at zero charge point for filtrate. It can be seen that when no fixative agent or flocculant was added, there is no fixation of microstickies and lignin. When only fixative agent polyDADMAC agent was added to neutralize the colloidal materials to zero charge point, the fixation of both the microstickies and lignin were just about $10 \%$. Only when flocculant was added, the fixation efficiency was significantly increased.

Figure 8 shows the retention efficiency of different flocculants without adding fixative agent. Results indicated that no water soluble lignin could be retented. CPAM could retent less than $5 \%$ of the microstickies, while PEO/PFR could retent $40 \%$ of the microstickies.

The results indicate the fixation of water-soluble anionic materials and microstickies needs the combination of fixative agent and flocculant. The fixative agent first neutralizes and destabilizes the water-soluble and colloidal contaminants, then the flocculant flocculates and rentents them on fiber web.

The effect of inorganic salts on fixation

In a closed mill, the salt concentration in the whitewater is normally high. Thus we studied the effect of salts on fixation. Figures 9 and 10 show the fixation efficiency of microstickies and lignin with polyDADMAC as fixative agent used with different flocculants at high salt concentrations. It can be seen that when CPAM was used as 
flocculant, the fixation efficiency of both microstickies and lignin was significantly reduced in high salt concentration. The maximum fixation rate for mirostickies was $60 \%$ and for lignin for $30 \%$. With PEO/PFR as flocculant, the fixation efficiency of microstickies was not effected by the salts, but the efficiency efficeincy for lignin was reduced to 50\%. Apparently salts affected not only the collision of microstickies, lignins, and also affected the conformation of flocculants. CPAM is more sensitive to high salt concentration, thus the retention efficiency is severely affected.

\section{Scanning Electronic Microscopy (SEM) results}

The fixation of colloidal contaminant was further studied by SEM. Lab made whitewater with colloidal particle size of 1.25 micron was used for the study. In $0.6 \%$ BSK pulp with 50 ppm (TOC) colloidal material was added various ammount of polyDADMAC, and stirred for 5 minutes. Fibers were taken out from the mixture and rinsed once in clean water, and then SEM samples were prepared. Figure 11 shows the SEM results. When no fixative agent is added, no colloidal materials were found on the fiber surface. When fixative agents were added, the colloidal particles gradually coagulated, part of them fixed on to fibers. Larger coagulates were formed near the zero charge point. When the fixative agent was overdosed, colloidal particles became cationic charged, and less coagulation happened. Some of the cationic charged particles fixed to fibers.

The results further indicated that the fixation process is actually happened after the coagulation process, and from previous results, retention agent is needed for effective fixation.

\section{Effect of microstickies and lignin fixation on paper properties}

It has been demonstrated that both lignin and microstickies can be effectively fixed to fibers with the combination of fixative agents and retention aids. We then investigated the effect of fixation on the possible effect of fixation on the paper physical properties. The results are shown in Figures 12-15. It was found that the fixation of microstickies 
has very small effect on the paper strength (tensile, burst and tear), and has no effect on optical property. The fixation of lignin increases the paper strength, and reduces the optical property.

\subsection{Removal of microstickies through flotation}

\section{Experimental}

Voith E-18 flotation cell was used for flotation study. Polyacrylate based pressuresensitive adhesive (B.F. Goodrich) was used as model sticky. The pulp furnish was $1 \%$ BSK pulp with 100 ppm PSA. PolyDADMAC is used as a charge neutralizer. Vinings A (a neutral surfactant, Vinings Industries) was used as surfactant. The experiment was conducted at $43{ }^{\circ} \mathrm{C}$. The content of the PSA was measured according to the following method: We assume all the materials in the pulp which can pass Whatmen 4 (size 25 microns) filter paper, but can not pass Mw 3000 cut-off membrane are microstickies. First the consistency of the pulp containing microstickies is determined through passing Whatmen 4 filter paper. Then certain amount of pulp is filtered. Part of the filtrate is filtered again through the Mw 3000 cut-off membrane. The Total Organic Carbon (TOC) in the two filtrates is measured, and thus the microstiky content in the filtrate is determined. The pulp cake is then dissolved with $0.5 \mathrm{M}$ cupric ethylenediamine according to the standard TAPPI Method T254 cm-85. The solution is then centrifuged and the residue is washed 3 times with DI water followed by centrifugation. The residue is dried and weighed, which is the microstickies in the cake. Added the microstickies in cak and in filtrate gives the total microsticky content.

\section{$\underline{\text { Results and Discussion }}$}

Figure 16 shows the results for the flotation removal of microstickies. The flotation time was 10 minutes. When only Vinings A, a commercial nonionic flotation deinking chemical for ONP deinking, was used for the flotation, the removal efficiency of the microstickies is about $66 \%$. The result is very similar to those obtained from previous 
flotation studies [4-6] for total stickies. If the microstickies were first neutralized by polyDADMAC (8 ppm in pulp furnish) before added Vinings A for flotation, the removal efficiency increased to 96\%. The PSA emulsion used has an average particle size of 190 $\mathrm{nm}$. It is know that flotation is less efficient to remove microparticles with size less than 10 microns. When polyDADMAC was added, the microstickies coagulated to form larger particles, and thus the removal efficiency increased significantly. PolyDaDMAC might have also changed the surface chemistry of the microstickies, and made the microstickies easier to attach to air bubbles. The results show that if the chemistry in deinking process is well controlled, most microstickies may be removed in flotation. Previous work [7] has indicated that charge neutralizer first reacted with the water soluble and colloidal anionic trash before reacted with fiber. But when the anionic materials are neutralized to near zero charge point, the competition between fiber and colloidal materials to react with the charge neutralizer will start. So if the charge is well controlled, the effect of charge neutralizer on fiber surface properties should be negligible.

\subsection{Conclusions}

Water-soluble and colloidal contaminants including microstickies can be fixed to fibers by flocculants in the presence of fixative agents around the iso-electrical point. Very high turbulence reduces the fixation efficiency. At high salt concentration, neutral flocculant is more effective than charged polymers for fixation. The fixation process is a process of neutralization, coagulation, fixation and retention. Fixation of microstickies has very small effect on the paper strength (tensile, burst and tear), and has no effect on optical property. Fixation of lignin increases the paper strength, and reduces the optical property. Microstickies can be effectively removed by flotation through charge control. The removal efficiency can be $96 \%$. Thus, cationic PSA may have extra benefit as a cationic fixation agent or neutralizer in paper recycling. 


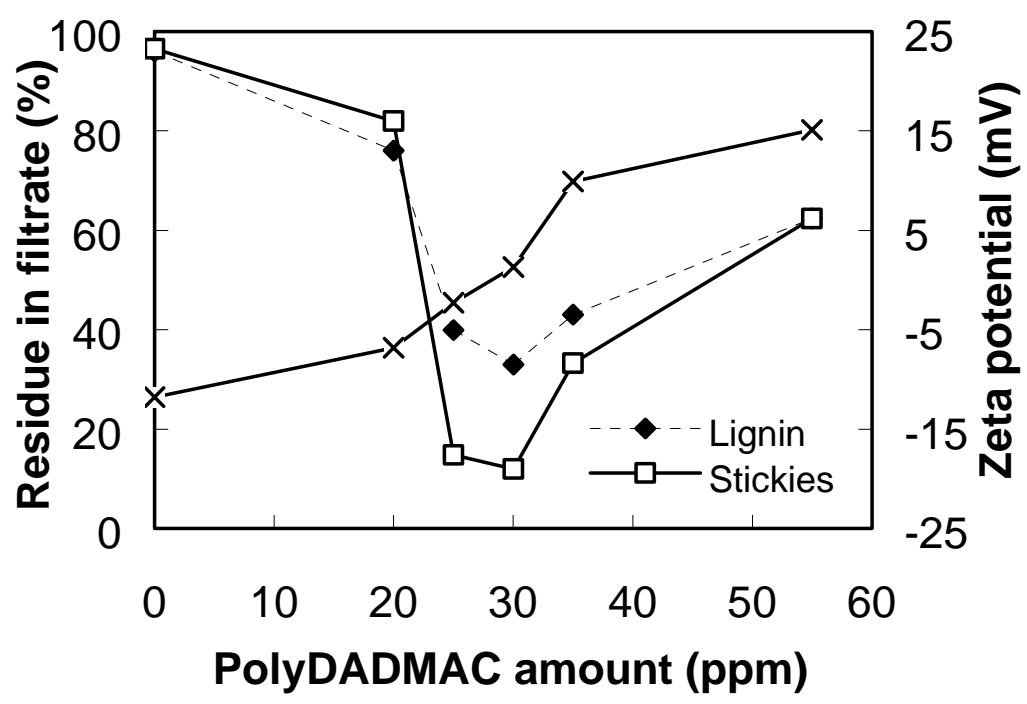

Figure 1. Fixation of stickies and lignin with polyDADMAC at pH 8.0. CPAM: 4ppm; stir rate $1000 \mathrm{rpm}$.

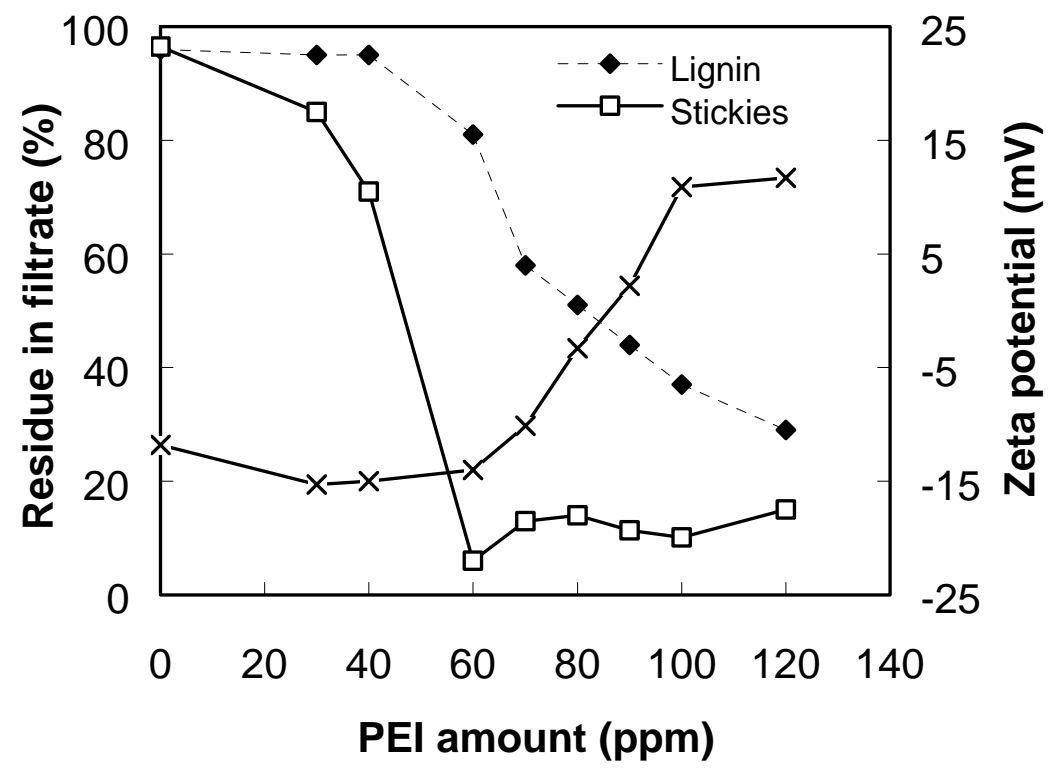

Figure 2. Fixation of stickies and lignin with PEI at pH 8.0. CPAM: 4ppm; stir rate $1000 \mathrm{rpm}$. 


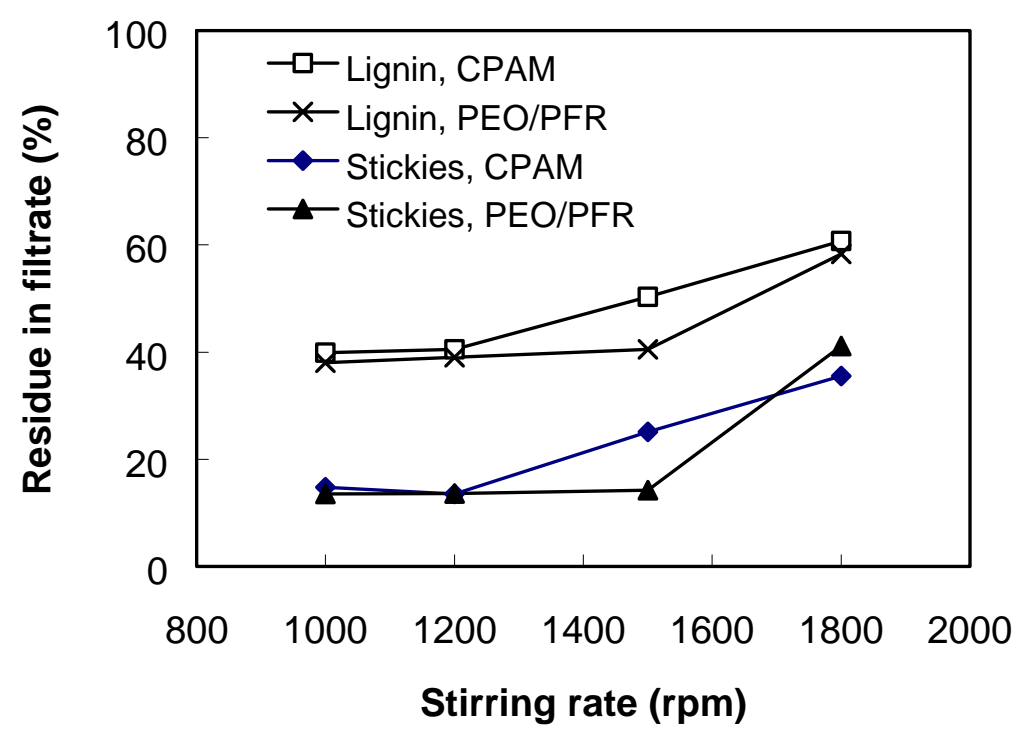

Figure 3. The effect of polyDADMAC molecular weight on fixation at $\mathrm{pH}$ 8.0. CPAM: 4ppm; stir rate $1000 \mathrm{rpm}$.

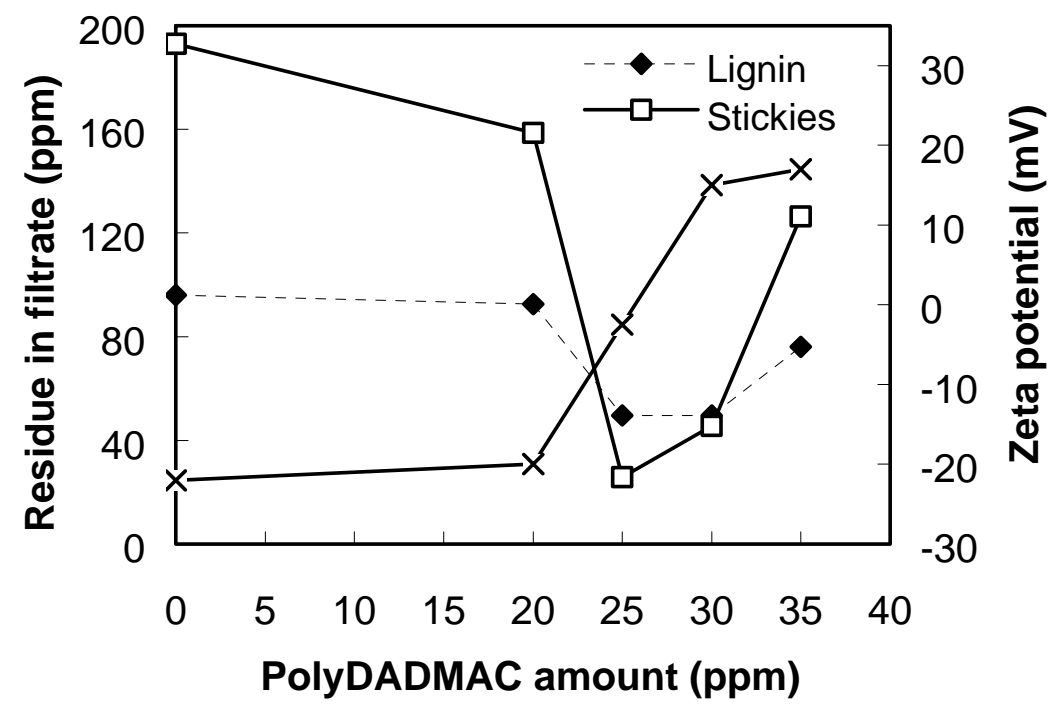

Figure 4. Fixation of stickies and lignin with polyDADMAC at pH 5.0. CPAM: 4ppm; stir rate $1000 \mathrm{rpm}$. 


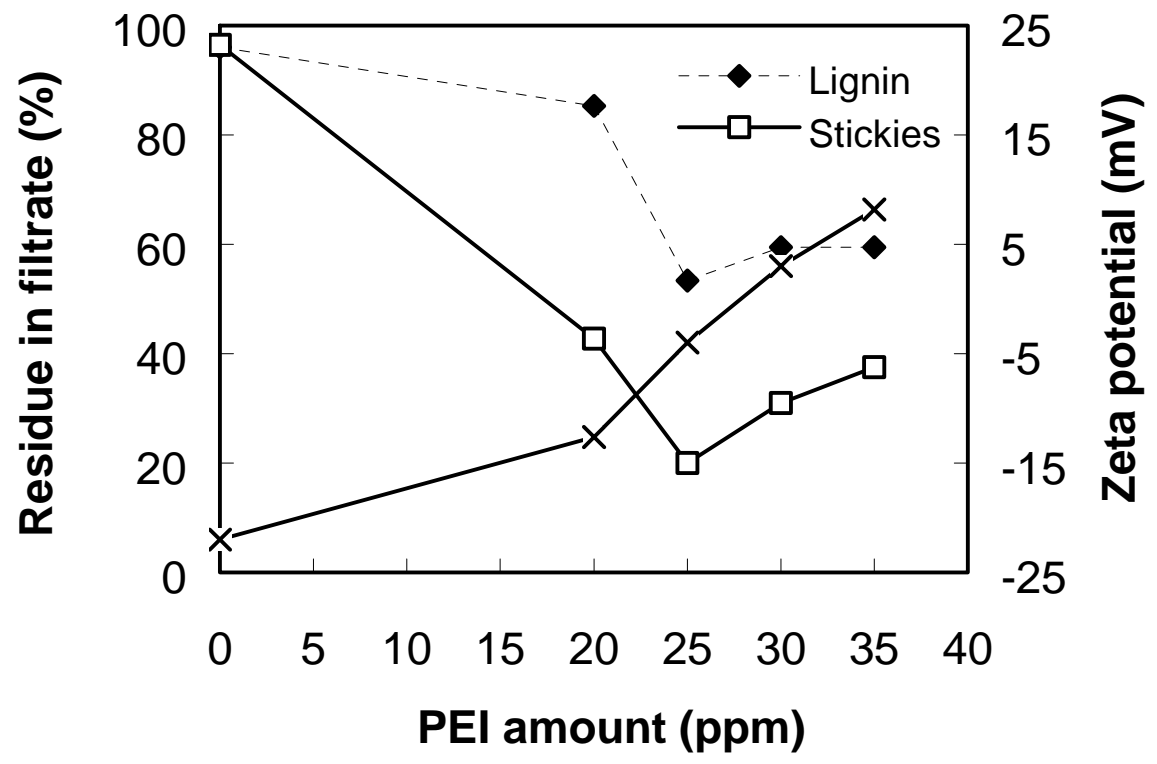

Figure 5. Fixation of stickies and lignin with PEI at pH 5.0. CPAM: 4ppm; stir rate 1000 rpm.

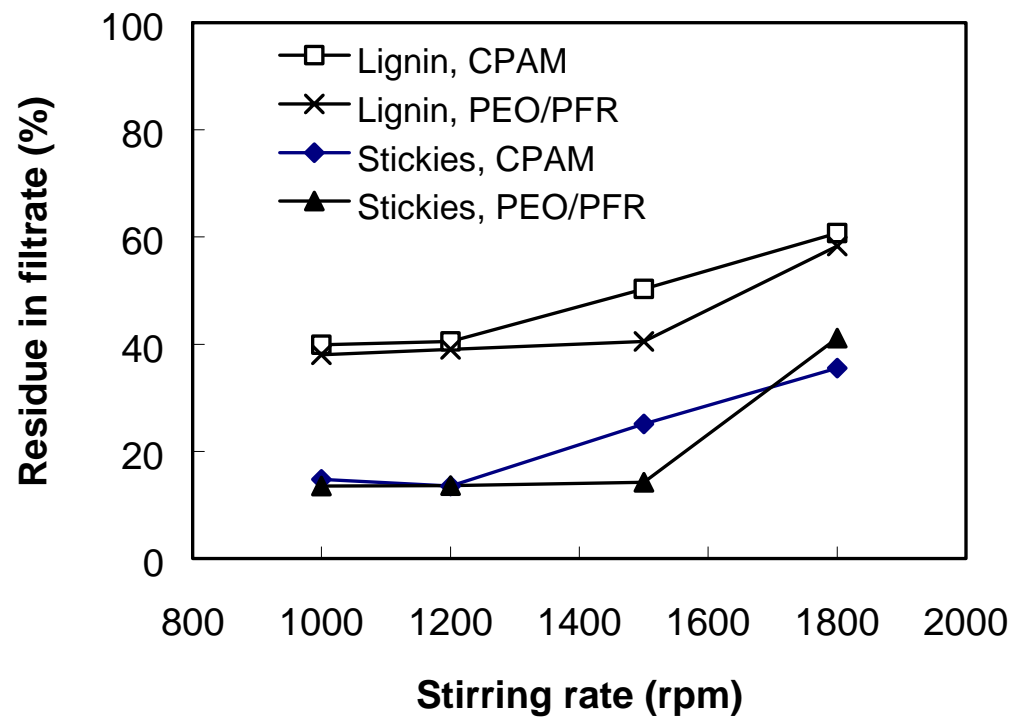

Figure 6. The effect of shear force on fixation with different flocculants at $\mathrm{pH}$ 8.0. CPAM: 4 ppm; PEO/PFR: 2 ppm/ 4 ppm. 


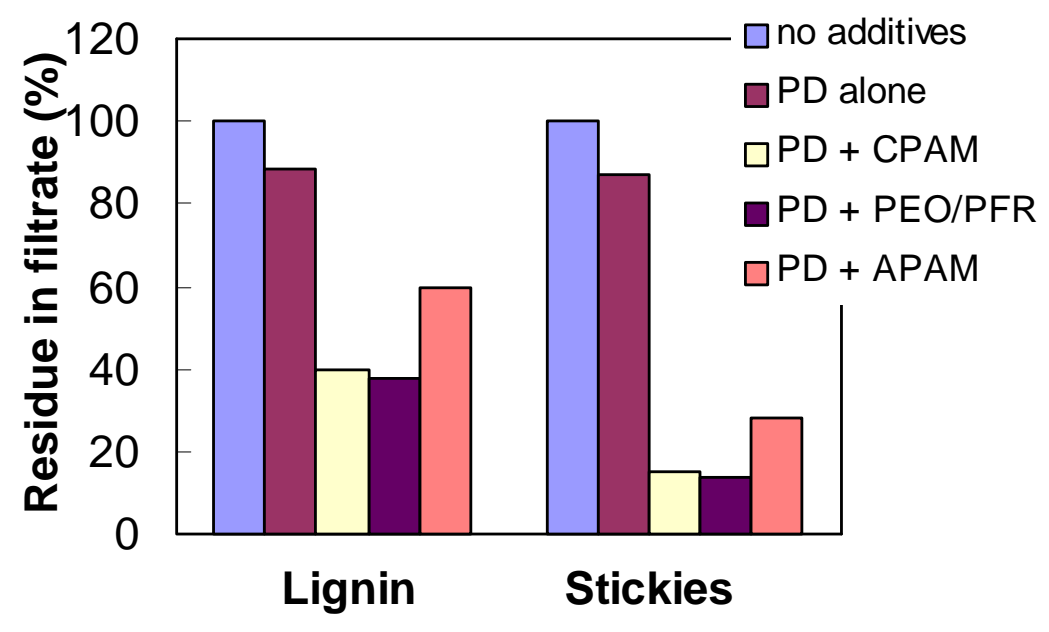

Figure 7. The effect of flocculant on fixation with fixative agent at $\mathrm{pH}$ 8.0. PolyDADMAC: 25 ppm; stir rate 1000 rpm.

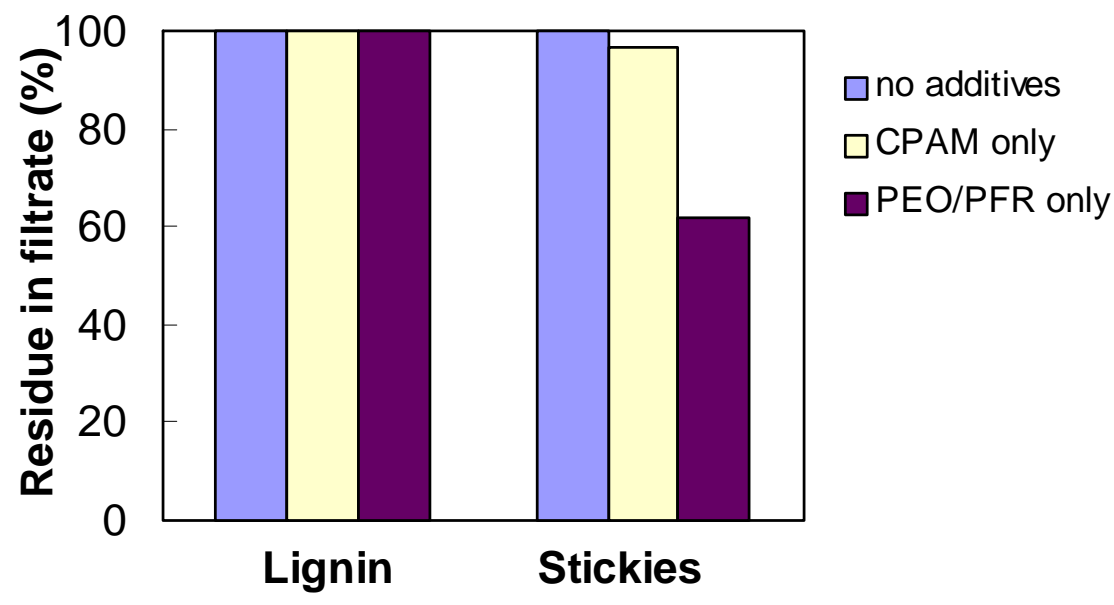

Figure 8. The effect of flocculant on fixation without fixative agent at $\mathrm{pH}$ 8.0. Stir rate $1000 \mathrm{rpm}$. 


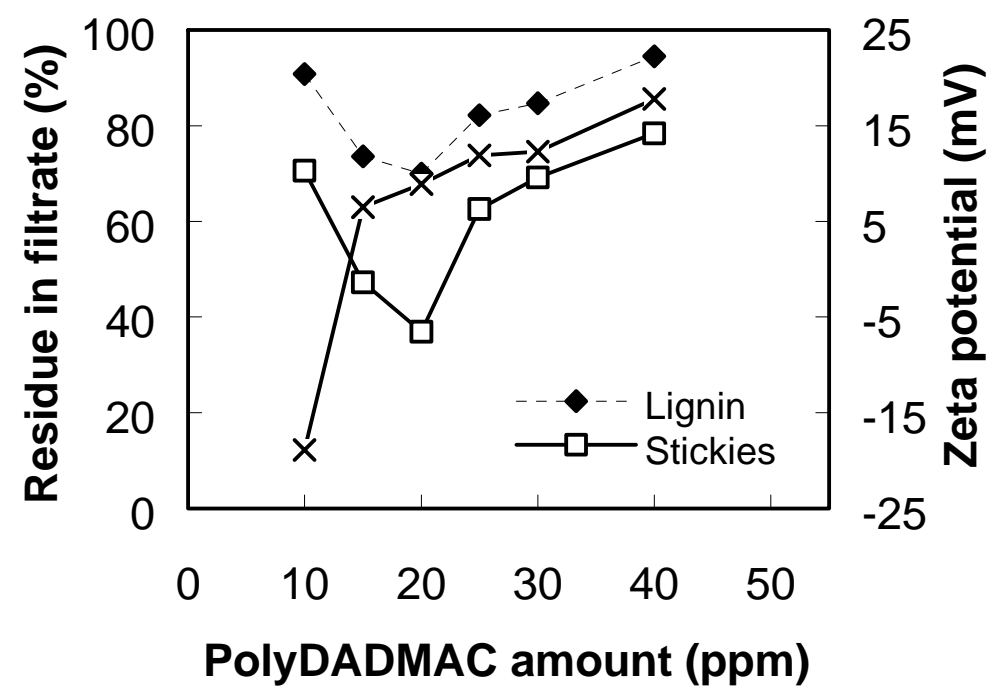

Figure 9. The effect of inorganic salts on fixation at $\mathrm{pH}$ 8.0. With CPAM: 4 ppm; stir rate $1000 \mathrm{rpm}$.

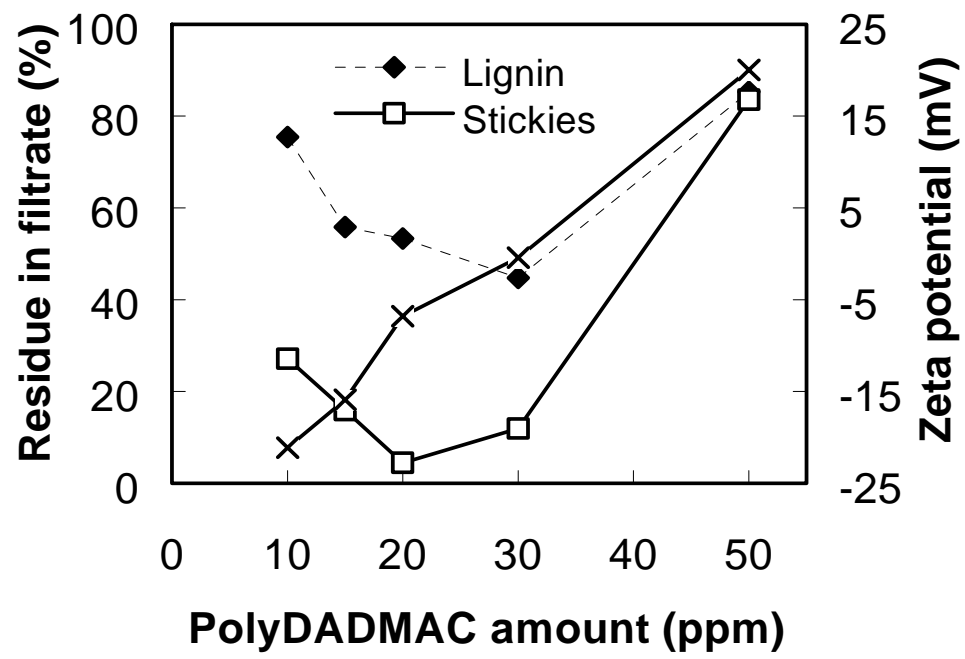

Figure 10. The effect of inorganic salts on fixation at $\mathrm{pH}$ 8.0. With PEO/PFR: $2 \mathrm{ppm} / 4$ ppm; stir rate $1000 \mathrm{rpm}$. 


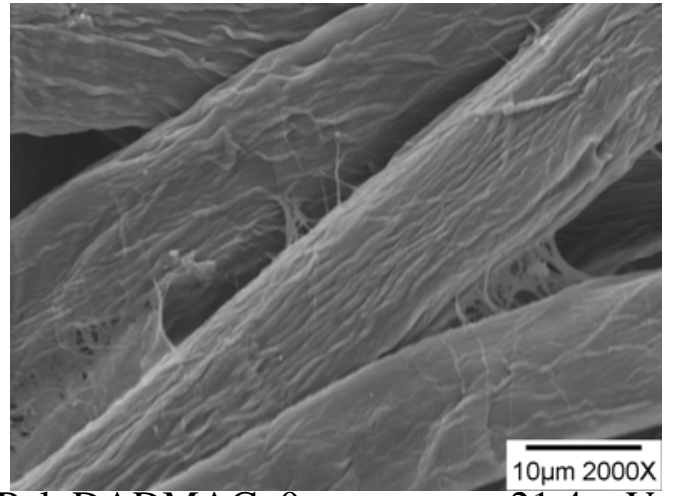

(a) PolyDADMAC: $0 \mathrm{ppm}$; zeta: $-21.4 \mathrm{mV}$

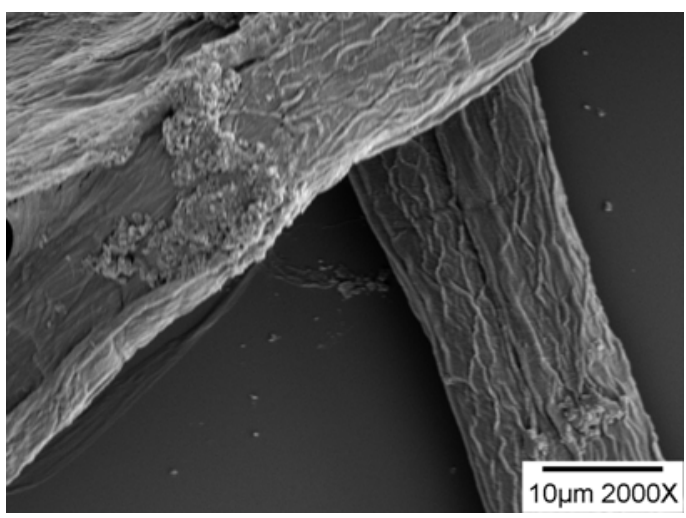

(c) PolyDADMAC: 5.0 ppm; zeta: $+0.5 \mathrm{mV}$

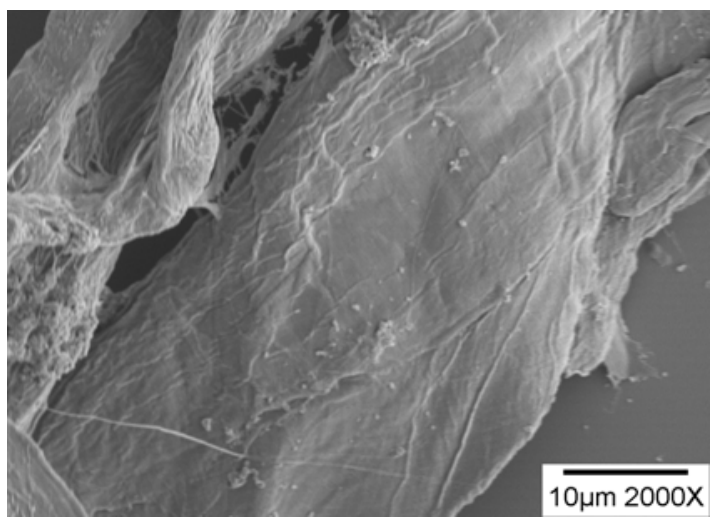

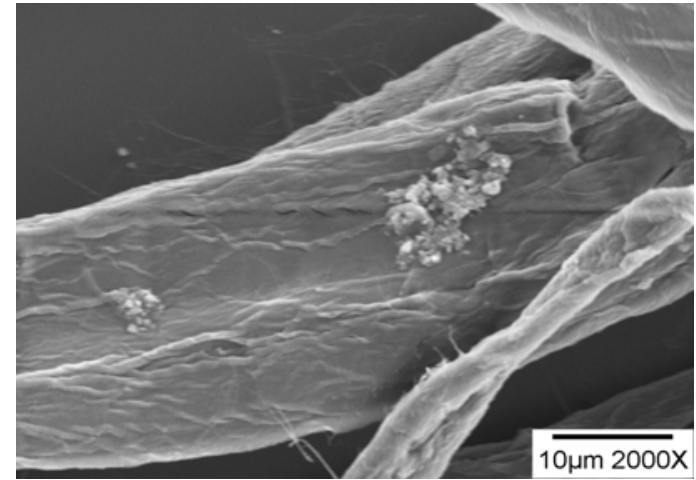

(b) PolyDADMAC: 2.5 ppm; zeta: $-7.5 \mathrm{mV}$

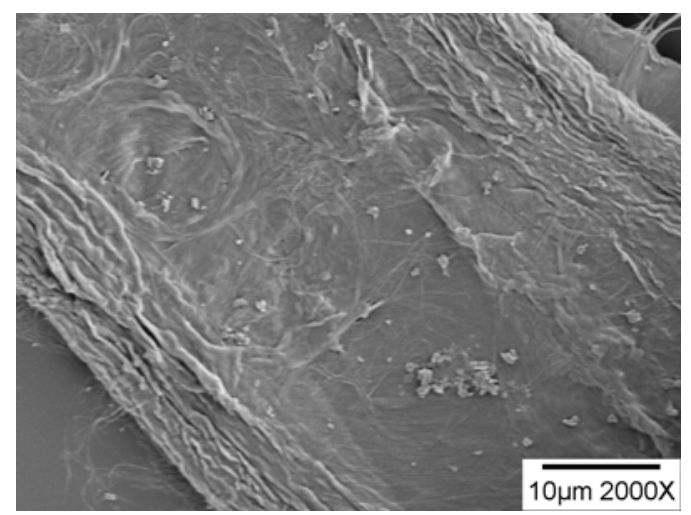

(d) PolyDADMAC: 7.5 ppm; zeta: $+13.1 \mathrm{mV}$

(e) PolyDADMAC: 10 ppm; zeta: $+17.0 \mathrm{mV}$

Figure 11. Scanning Electron Microscopy (SEM) of microstickies on fibers with different amount of fixative agent polyDADMAC. 


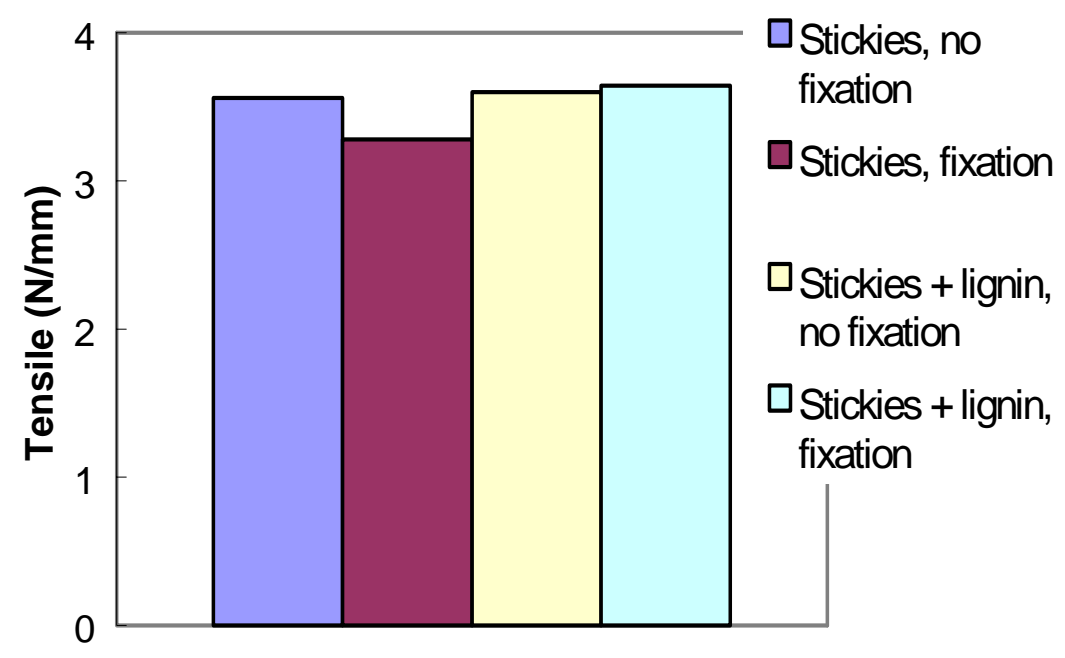

Figure 12. The effect of stickies and lignin fixation on paper tensile strength.

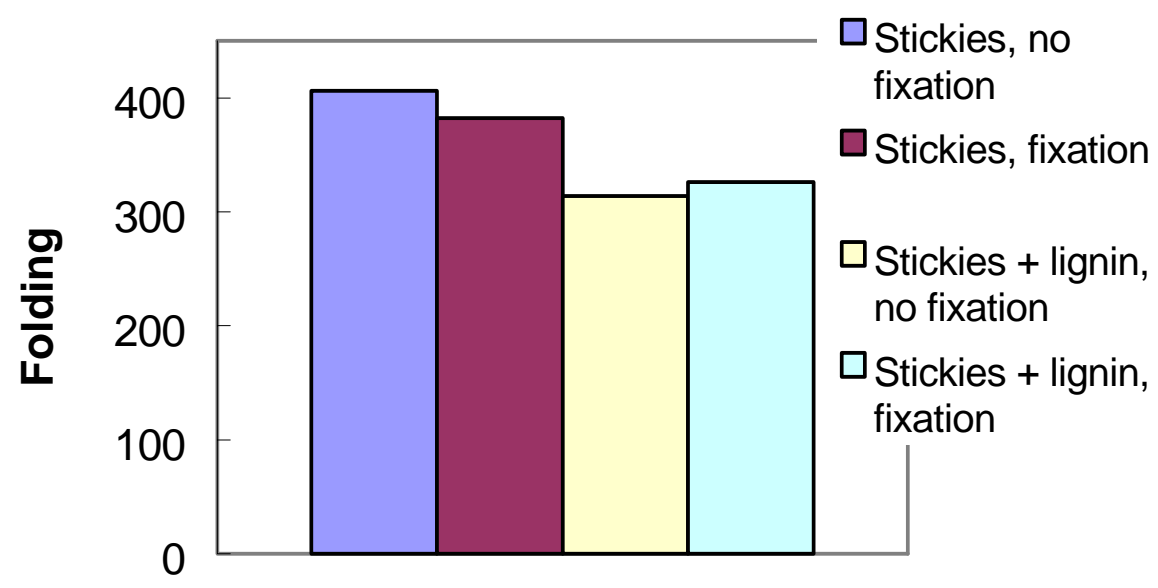

Figure 13. The effect of stickies and lignin fixation on paper folding strength. 


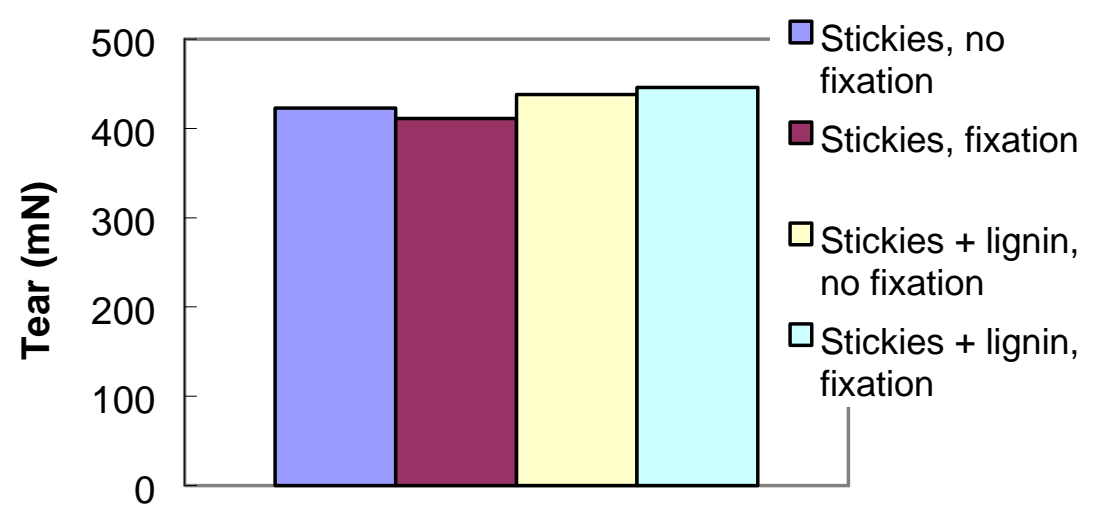

Figure 14. The effect of stickies and lignin fixation on paper tear strength.

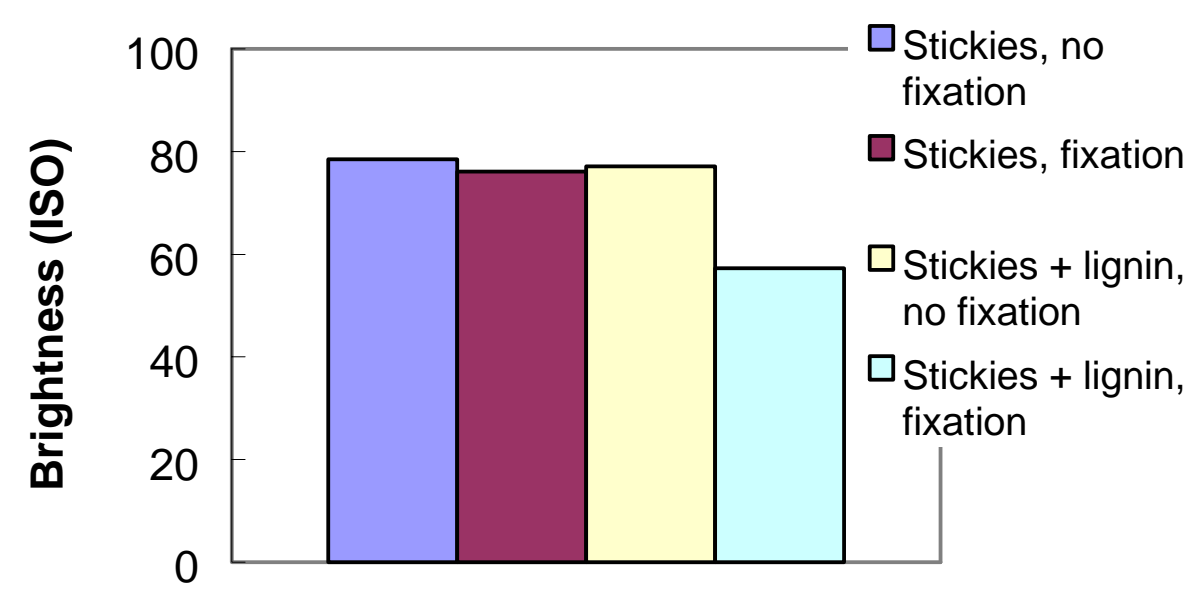

Figure 15. The effect of stickies and lignin fixation on paper optical property. 


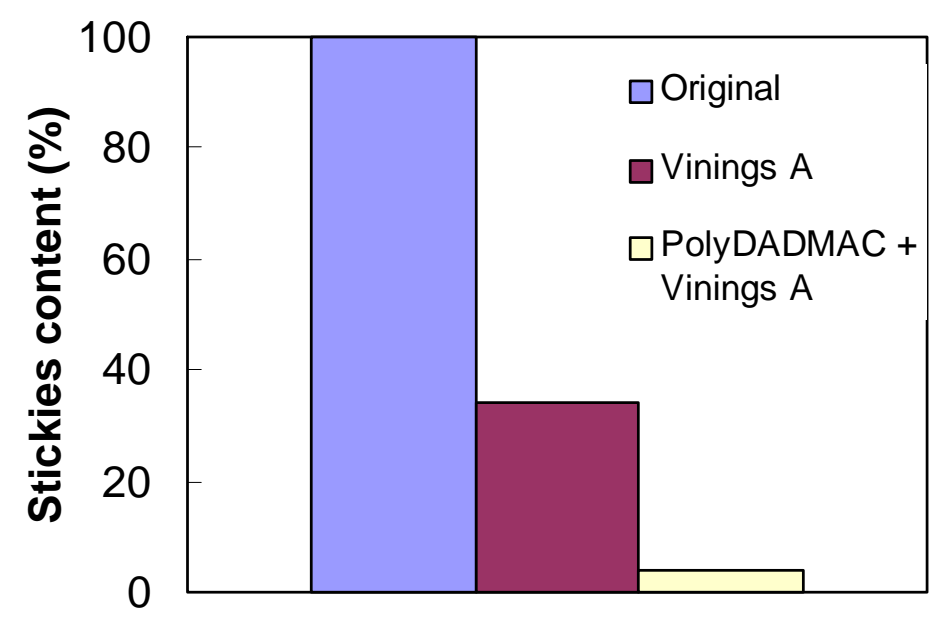

Figure 16. Removal of microstickies through flotation.

\section{References}

1. T. Woodward, Proc. Waste Paper VII, 4, Chicago, IL, June (1996).

2. D.J.H. Wenzl, Proc. 1981 TAPPI Annual Meeting, TAPPI Press, P95.

3. F.J.S. Amand, B. Perrin and P. de Luca, Prog. Paper Recy., 39, August (1998).

4. N.N.-C. Hsu and D.L. Dauplaise, Proc. TAPPI Recycling Symp., 249, TAPPI Press, Atlanta, GA, March (1996).

5. L. Warberg and L. Odberg, Nordic Pulp Paper Res. J., 6(3), 127(1991).

6. M. Geistbeck and H. Wiese, Twogether Pap. Technol. J., 4, 30 (1997).

7. Y. Deng and Y. Xu, "Wet-End Chemistry in Highly Contaminated Systems", Status Report for IPST Project F043, February (2001). 